\title{
Effective forecasting of stock market price by using extreme learning machine optimized by PSO-based group oriented crow search algorithm
}

\author{
Sudeepa Das ${ }^{1}$ (]) $\cdot$ Tirath Prasad Sahu ${ }^{1} \cdot$ Rekh Ram Janghel $^{1} \cdot$ Binod Kumar Sahu ${ }^{2}$ \\ Received: 16 October 2020 / Accepted: 31 July 2021 / Published online: 13 August 2021 \\ (C) The Author(s), under exclusive licence to Springer-Verlag London Ltd., part of Springer Nature 2021
}

\begin{abstract}
Stock index price forecasting is the influential indicator for investors and financial investigators by which decision making capability to achieve maximum benefit with minimum risk can be improved. So, a robust engine with capability to administer useful information is desired to achieve the success. The forecasting effectiveness of stock market is improved in this paper by integrating a modified crow search algorithm (CSA) and extreme learning machine (ELM). The effectiveness of proposed modified CSA entitled as Particle Swarm Optimization (PSO)-based Group oriented CSA (PGCSA) to outperform other existing algorithms is observed by solving 12 benchmark problems. PGCSA algorithm is used to achieve relevant weights and biases of ELM to improve the effectiveness of conventional ELM. The impact of hybrid PGCSA ELM model to predict next day closing price of seven different stock indices is observed by using performance measures, technical indicators and hypothesis test (paired $t$-test). The seven stock indices are considered by incorporating data during COVID-19 outbreak. This model is tested by comparing with existing techniques proposed in published works. The simulation results provide that PGCSA ELM model can be considered as a suitable tool to predict next day closing price.
\end{abstract}

Keywords Metaheuristic Algorithm · Extreme Learning Machine $\cdot$ Stock indices forecasting $\cdot$ Modified sharpe ratio

\section{Introduction}

Stock market (SM) provides a path for companies to make profit by allowing businesses traded publically which enhance the financial capital for expansion by selling shares of the company in public market. The objective of investment in SM is to raise revenue through purchasing

Sudeepa Das

sdas.phd2019.it@nitrr.ac.in

Tirath Prasad Sahu

tpsahu.it@nitrr.ac.in

Rekh Ram Janghel

rrjanghel.it@nitrr.ac.in

Binod Kumar Sahu

binodsahu@soa.ac.in

1 Department of Information Technology, National Institute of Technology, Raipur, Chhattisgarh, India

2 Department of Electrical Engineering, ITER, Siksha 'O' Anusandhan University, Bhubaneswar, Odisha, India and holding a portfolio of stocks, mutual funds, bonds and other instruments. In last few decades, the investors and traders experience huge risk to invest in stock portfolios because of its inconsistency and variations. The inconsistency in SM occurs due to the factors such as economic condition, political activities and trader's prediction. From the fluctuating market, traders always try to make transactions over a small time frame to achieve frequent profit. The key of stock traders to get maximum profits with less risk is conceivable by establishing an accurate trading decision making tool with respect to time. The future price is predicted with better accuracy by concerning the patterns of historical data (price and volume) [1].

So, the prediction of closing price of highly dynamic and nonlinear SM is very indispensable. Auto Regressive Integrated Moving Average (ARIMA) model is used to interpret the time series data and to forecast the future in that series [2]. But, this conventional technique is not adequate enough to forecast the SM price whose movements are influenced by some macro-economic factors [3, 4]. Singh et al. [5] have proposed a hybrid wavelet 
denoise-ARIMA model to improve the prediction accuracy of stock market price. The close prediction of SM helps investors to draw better benefits from SM. In this work, optimized ELM model is developed to capture the hidden structural changes in volatility processes of portfolio returns.

Some researchers have proposed decision making tools based on artificial intelligence (AI) and deep learning techniques to enhance the accuracy of the SM forecasting. Bustos et al. [6] have contributed a brief survey of different methods implemented to predict stock market price. Artificial neural network (ANN)-based model is preferred to forecast SM [7-10], because of its benefits such as organic learning, nonlinear data processing, fault tolerance and self-overhaul. Feedforward neural network (FFNN) is a simple form of ANN, which has very less computational time to solve simple problems with less complexity [11]. The effectiveness of deep feedforward neural network to forecast stock indices price is analysed by a comparative analysis with ANN [12]. Multilayer perceptron (MLP) is a persuasive ANN to be used for regression and is efficient enough to predict SM price [13]. Back propagation neural network (BPNN) is also implemented to solve SM index forecasting problem $[14,15]$. Convolutional neural network (CNN) is a fully connected MLP, which facilitates less computational complexity without defeating the substance of the data. The improvement analysis of CNN to predict intraday price forecasting is studied in [16, 17]. From last few decades, different forms of ANN are accepted in the field of SM forecasting. All of these forms of ANN are gradient-based algorithms with certain constraints such as high computational time to train and probability to get trapped in local optima of the problem. The dilemma of ANN is overwhelmed by support vector machines (SVM) to predict time series data [18]. A comparative analysis between SVM and Adaptive Neuro Fuzzy Inference System (ANFIS) in terms of finger-vein identification is portrayed and the SVM is concluded as a superior technique over ANFIS with less computational time and robust classifier [19]. Further, the efficacy of SVM to forecast stock market price is improved by integrating piecewise linear representation with weighted SVM and optimized SVM techniques in [20] and [21] respectively. Long short-term memory (LSTM) is validated over ANN and SVM to predict stock index [22]. Huang et al. [23] have proposed a Single-hidden Layer Feed forward Neural Network (SLFNN) entitled as ELM. It is required to regulate the number of neurons \& activation function of ELM as the input weights \& hidden layer biases are fixed during the employment. These properties of ELM enhance the notoriety to contribute better generalization performance with immensely rapid learning. Cheng et al. [24] have beautifully conferred the supremacy of ELM over SVM to predict petroleum reservoir permeability. Huang et al. [25] have implemented ELM for regression and classification in various fields. From previous decade, ELM is considerably demonstrated the superiority over traditional techniques in the field of SM forecasting [26, 27].

Some researchers are considered the optimization techniques to boost the efficiency of existing machine learning techniques to predict SM with superior accuracy. Genetic algorithm (GA)-based fuzzy [28], SVM [29], ANN [30], multi-chanel CNN [31] and Probabilistic Weight Support Vector Machine (PWSVM) [32] are flourishingly implemented to forecast SM price, return and trend with improved accuracy. Hegazy et al. [33] have applied particle swarm optimization (PSO) optimized ANN and Least Square Support Vector Machine (LS-SVM), respectively,

Fig. 1 Structure of ELM

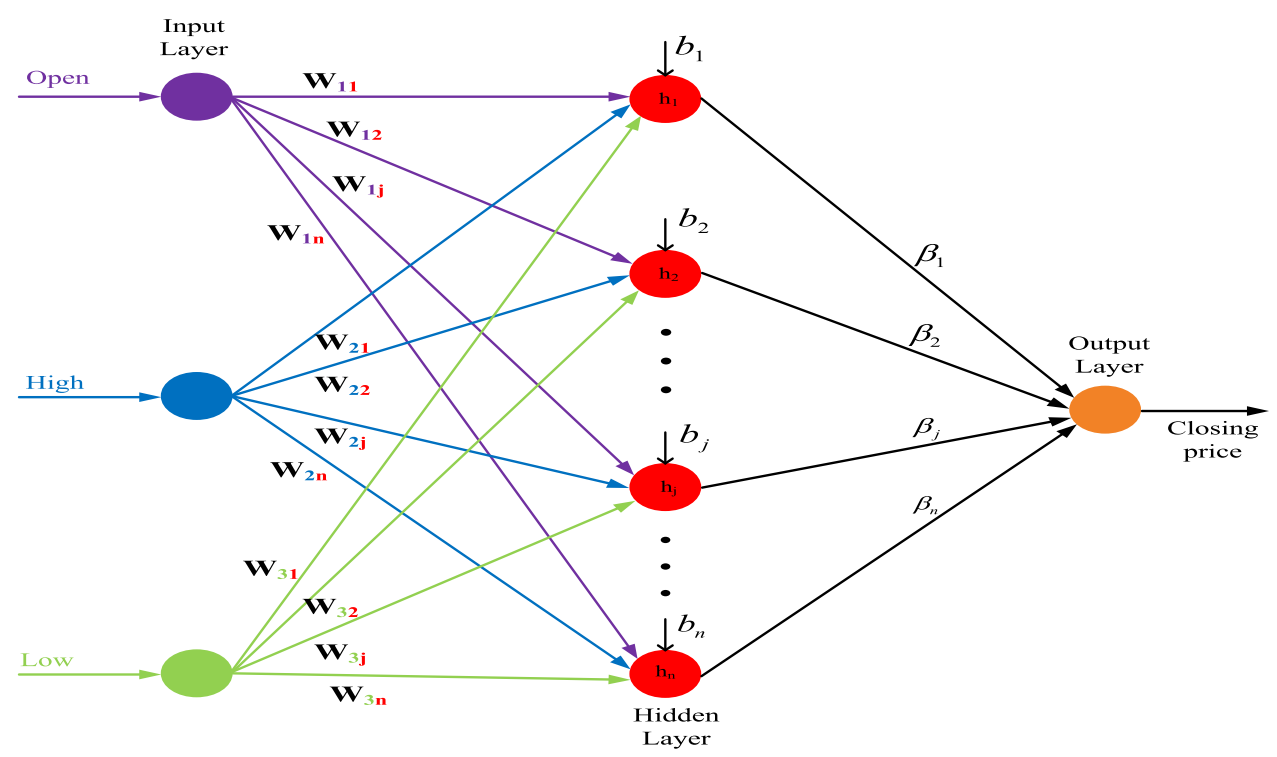




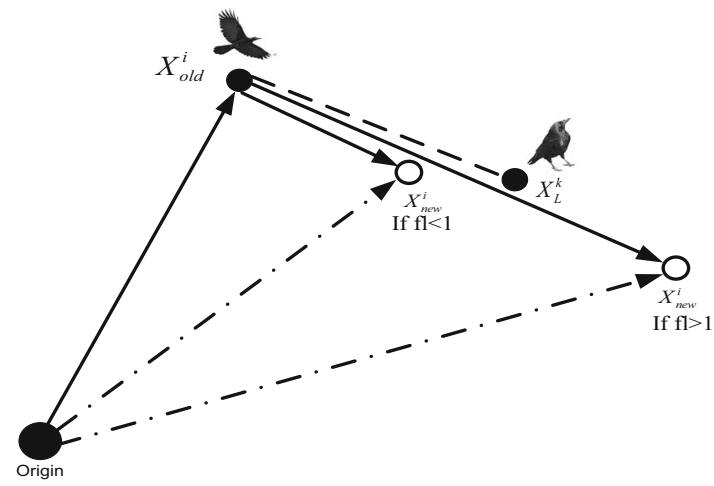

(a)

Fig. 2 Crow position updating $\mathbf{a}$ if $r_{1} \geq \mathrm{AP}$ and $\mathbf{b}$ if $r_{1}<\mathrm{AP}$

to predict SM. Computationally Efficient Functional Link Artificial Neural Network (CEFLANN) optimized by Differential Evolution (DE) algorithm is implemented with better performance to predict SM [34]. The accuracy of stock index forecasting is enhanced by Artificial Fish Swarm Algorithm (AFSA)-based Radial Basis Functional Neural Network (RBFNN) and Grey Wolf optimizationbased Elman neural network proposed by Shen et al. [35] and Chander [36] respectively. SM price forecasting problem is solved with improved accuracy by implementing chaotic Firefly Algorithm (FA)-based Support Vector Regression (SVR) [37] and Discrete PSO (DPSO)-based Fully Complex-valued Radial Basis Functional Neural Network (FCRBFNN) [38]. Hybrid Artificial Bee ColonyDifferential Evolution (ABC-DE) is applied to optimize weights of Feed Forward Neural Network (FFNN) to predict foreign exchange rate [39].

The conventional techniques may not efficient enough to solve high dimension multimodal objective function. Because of the flexibility and gradient free mechanism, metaheuristic techniques are mostly preferred methods to deal with high dimension, nonlinear, complex and multimodal problems. Evolutionary based, physics based, swarm based and nature inspired algorithms are the most well-known categories of metaheuristic algorithms [40]. Genetic Algorithm (GA) [41], Differential Evolution (DE) [42], Gravitational Search Algorithm (GSA) [43], Particle Swarm Optimization (PSO) [44], Artificial Bee Colony (ABC) [45], Grasshopper Optimisation Algorithm (GOA) [46] and Symbiotic Organism Search (SOS) [47] etc. are the most desirable optimization techniques under these categories mentioned above. CSA is a recent metaheuristic algorithm proposed by Askarzadeh [48]. This algorithm is derived by the social behaviour of quick witted and clever organism of ecosystem. By doing a team work, crows perform incredible sample of brilliance and gain good results. The techniques used by crows to memorizing and

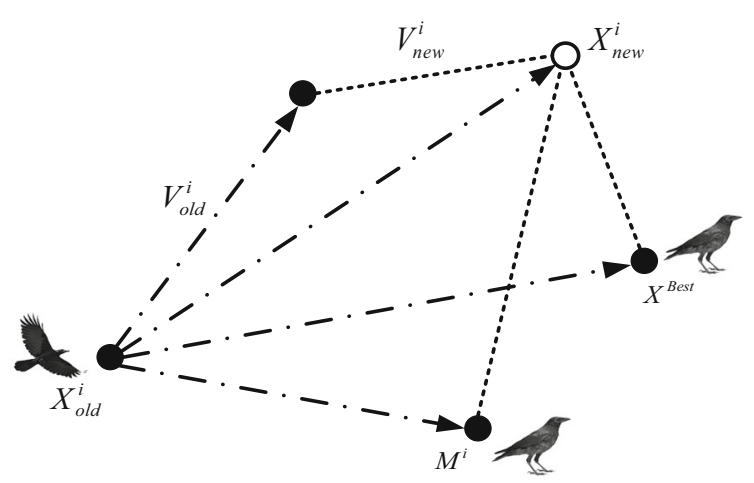

(b)

recognizing faces are unique. The sluggish convergence rate and chance of got stuck in local optima are the considerations which motivate researchers to overcome the dilemma. Chaotic maps are implemented in CSA to overcome this dilemma and the improvement is realized favourably by solving engineering and constrained problems [49, 50]. The 'flight length' of CSA is made adaptive iteratively and applied in economic load dispatch problem [51]. The hybridization of CSA with Rough Searching Scheme (RSS) and Sine Cosine Algorithm (SCA) algorithms are endorsed to enhance the proficiency of individual algorithms [52, 53].

From the survey, ELM technique is established as an adequate and admirable technique to predict time series data. The weights and biases of ELM are an influential aspect to enhance the performance. In this paper, a strive approach is introduced to enhance the potential of CSA entitled as PGCSA. CSA and PGCSA algorithms are used to optimize the weights and biases of ELM for forecasting different SMs price. PGCSA ELM is concluded as a superior technique by contributing a fine comparative analysis with some published paper MLP, GARCH-DAN2 and BNNMAS techniques [10, 54].

Contributions of the article are as follows:

i. Optimized ELM (with optimized weights and biases) is proposed to forecast price of seven distinct stock markets.

ii. Two phases (with and without awareness probability) of CSA is altered to enhance the searching capability of CSA algorithm entitled as PGCSA.

iii. PGCSA and CSA algorithms are substantiated over some recently published papers by resolving benchmark equations and hypothetically tested by using Wilcoxon signed-rank test.

iv. Comparative analysis of PGCSA-ELM with CSAELM and ELM (randomly fixed weights and biases). The proposed model predicted closing price is tested 


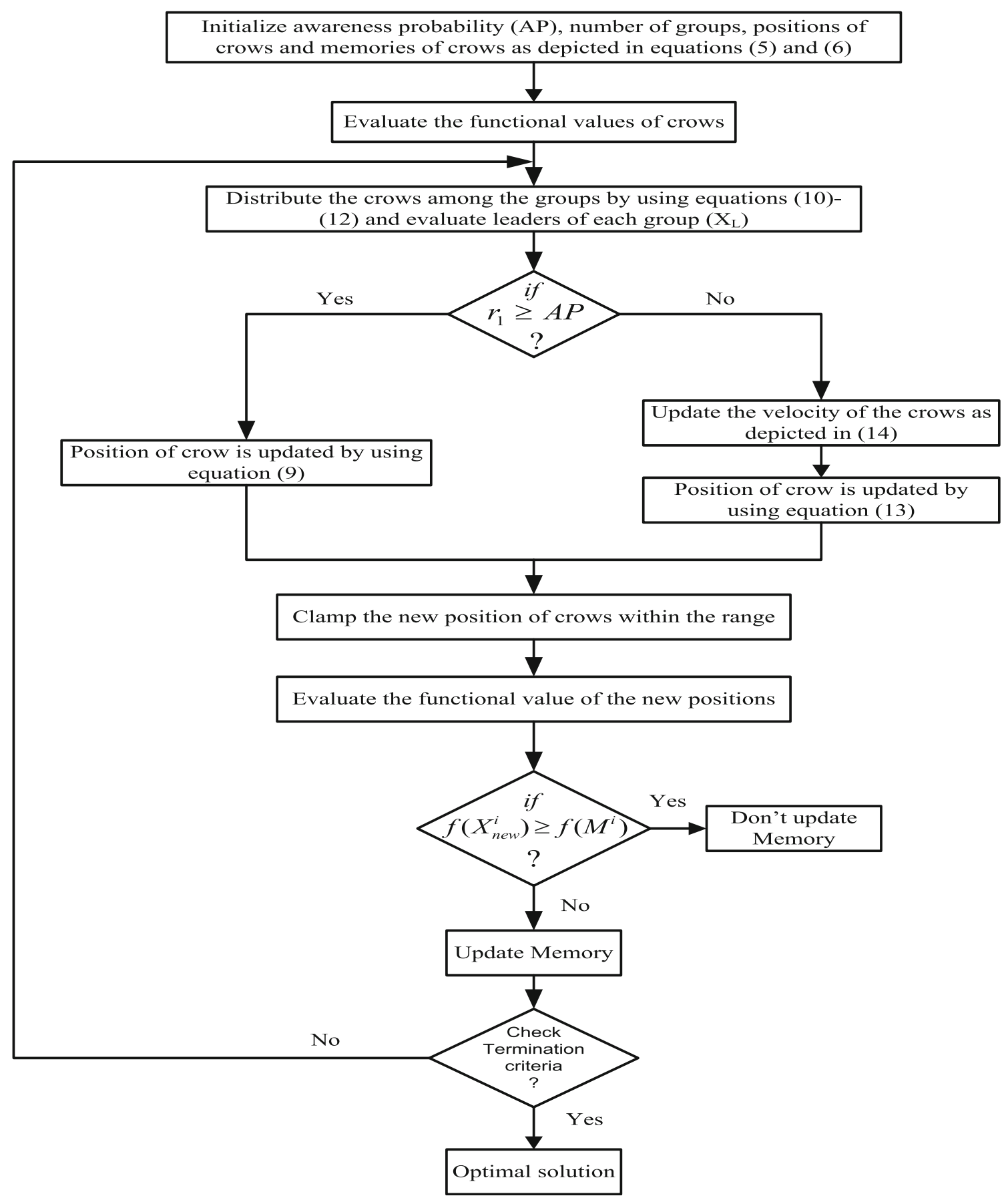

Fig. 3 Flow chat of PGCSA algorithm

by using some technical indicators such as MSE, MAE, MAPE, MAAPE, CoV, CORR and Theil's U of SM forecasting.

v. The accuracy of predicted closing price is tested hypothetically by using paired t-test and by using financial indicators such as sharpe ratio, and modified sharpe ratio.

\section{Methodology and data}

\subsection{Extreme learning machine (ELM)}

Huang et al. [23] have introduced a SLFNN entitled as ELM. Input, hidden and output layers are the basic segments of ELM. The hidden layer neurons are not to be optimized during training period. These neurons are distributed arbitrarily and never refurbished. Inputs are linked 
Table 1 Benchmark Equations (Unconstraint)

\begin{tabular}{llllll}
\hline No & Functions & Range & D & Formulation & $\mathrm{f}_{\text {min }}$ \\
\hline 1 & Beale & {$[-4.5,4.5]$} & 2 & $f(x)=\left(1.5-x_{1}+x_{1} x_{2}\right)^{2}+\left(2.25-x_{1}+x_{1} x_{2}^{2}\right)^{2}+\left(2.625-x_{1}+x_{1} x_{2}^{3}\right)^{2}$ & 0 \\
2 & Six Hump Camel Back & {$[-5,5]$} & 2 & $f(x)=4 x_{1}^{2}-2.1 x_{1}^{4}+\frac{1}{3} x_{1}^{6}+x_{1} x_{2}-4 x_{2}^{2}+4 x_{2}^{4}$ & -1.0316 \\
3 & Zakharov & {$[-5,10]$} & 10 & $f(x)=\sum_{i=1}^{D} x_{i}^{2}+\left(\sum_{i=1}^{D} 0.5 i x_{i}^{2}\right)^{2}+\left(\sum_{i=1}^{D} 0.5 i x_{i}^{2}\right)^{4}$ & 0 \\
4 & Step & {$[-5.12,5.12]$} & 30 & $f(x)=\sum_{i=1}^{D}\left(x_{i}+0.5\right)^{2}$ & 0 \\
5 & Sphere & {$[-100,100]$} & 30 & $f(x)=\sum_{i=1}^{D} x_{i}^{2}$ & 0 \\
6 & Rastrigin & {$[-5.12,5.12]$} & 30 & $f(x)=\sum_{i=1}^{D}\left(x_{i}^{2}-10 \cos \left(2 \pi x_{i}\right)+10\right)$ & 0 \\
7 & Griewank & {$[-600,600]$} & 30 & $f(x)=\frac{1}{4000}\left(\sum_{i=1}^{D}\left(x_{i}-100\right)^{2}\right)-\left(\prod_{i=1}^{D} \cos \left(\frac{x_{i}-100}{\sqrt{i}}\right)\right)+1$ & 0 \\
8 & Ackley & {$[-32,32]$} & 30 & $f(x)=-20 \exp \left(-0.2 \sqrt{\frac{1}{\pi} \sum_{i=1}^{D} x_{i}^{2}}\right)-\exp \left(\frac{1}{\pi} \sum_{i=1}^{D} \cos \left(2 \pi x_{i}\right)\right)+20+e$ & -959.6407 \\
9 & Egg Holder & {$[-512,512]$} & 2 & $f(x)=-\left(x_{2}+47\right) \sin \left(\sqrt{\left|x_{2}+\frac{x_{1}}{2}+47\right|}\right)-x_{1} \sin \left(\sqrt{\left|x_{1}-\left(x_{2}+47\right)\right|}\right)$ \\
\hline
\end{tabular}

to hidden layers with randomly fixed weights $\left(\mathrm{w}_{\mathrm{i}}\right)$ and the biases $\left(b_{j}\right)$. In this work, CSA and PGCSA algorithms are administrated to search convenient weights and biases of the ELM. The structure of ELM is illustrated in Fig. 1. ELM has immense convergence which is thousand time faster than BPNN [25]. Non-gradient-based ELM has not only better generalized performance over gradient-based techniques but also avoid the local minima, erroneous learning rate and over fitting. The output function of ELM with $\mathrm{L}$ hidden nodes for a training set $\mathbf{R}=\left\{\left(\mathbf{X}_{\mathbf{i}}, \mathbf{t}_{\mathbf{i}}\right)\right\}, \mathbf{i}=$ $1,2, \ldots, \mathbf{n}$ is illustrated in Eq. (1).

$f(R)=\sum_{j=1}^{L} \beta_{j} H(X)=t_{j}$

where, $\beta=\beta_{1}, \beta_{2}, \ldots, \beta_{\mathrm{L}}$ is the weight matrix between hidden and output layer and $t=t_{1}, t_{2}, \ldots, t_{\mathrm{j}}$ is the target matrix of training data. The output of hidden layer is estimated by Eq. (2).

$H=\left[\begin{array}{ccc}G\left(w_{1}, b_{1}, X_{1}\right) & \cdots & G\left(w_{L}, b_{L}, X_{1}\right) \\ G\left(w_{1}, b_{1}, X_{2}\right) & \cdots & G\left(w_{L}, b_{L}, X_{2}\right) \\ \vdots & \vdots & \vdots \\ G\left(w_{1}, b_{1}, X_{n}\right) & \cdots & G\left(w_{L}, b_{L}, X_{n}\right)\end{array}\right]$

$\beta=\left[\begin{array}{c}\beta_{1} \\ \beta_{2} \\ \vdots \\ \beta_{L}\end{array}\right]=\left(H^{T} H\right)^{-1} H^{T}$

" $G$ " is the activation function in terms of weight, bias and inputs. Sigmoidal, Gaussian, Hard limit and Fourier series functions are adopted as activation functions. In this work, sigmoidal function is adopted as activation function as illustrated in Eq. (3).
$G\left(w_{i}, b_{i}, X_{i}\right)=\frac{1}{1+e^{-(w x+b)}}$

The desired output of the ELM is determined by using Eq. (4).

$T_{\text {test }}=H \beta$

The ELM is trained by considering intraday open, high $\&$ low prices of stock indices as inputs and closing price as target. The closing price of stock market is predicted by conceding open, high $\&$ low prices. The weight matrix $\left(\mathrm{w}_{\mathrm{ij}}\right)$ and bias $\left(\mathrm{b}_{\mathrm{j}}\right)$ are optimized by CSA and PGCSA algorithms to enhance the forecasting capability of ELM. The performance of the forecasting capability of ELM is graded by the performance measure such as MSE (Mean Squared Error).

\subsection{Crow search algorithm (CSA)}

CSA algorithm is derived from the social behavior of crows [48]. Crows are opted as the ultimate brilliant bird. Compared to their physical structure they have tremendous brain. Crows conceal their overabundance food in undoubted location and when it is essential they recoup it. Crows acquire food by doing a great team work always. Hiding foods for the next season is not that much easy for a crow because some opponent crows can also come after to follow the food. At that moment, the crow endeavors to mislead by changing the direction in the territory. Here the crows can be taken as path finders or searches whereas the territory can be taken as search area. Every location of the territory is considered as optimum point and the fitness value is the quality of the nourishment source. Crows steal the hiding nourishment of other birds by observing and following them and at the same time the crows take some 
Table 2 Benchmark Equations (Constraint)

\begin{tabular}{|c|c|c|c|c|c|}
\hline No & Functions & Range & $\mathrm{D}$ & Formulation & $f_{\min }$ \\
\hline 10 & Rosenbrock (disk) & $\begin{array}{l}-1.5 \leq x_{1} \leq 1.5 \\
-1.5 \leq x_{2} \leq 1.5\end{array}$ & 2 & $\begin{array}{l}f(x)=\left(1-x_{1}\right)^{2}+100\left(x_{2}-x_{1}^{2}\right)^{2} \\
\text { subjectedto }: x_{1}^{2}+x_{2}^{2} \leq 2\end{array}$ & 0 \\
\hline 11 & Mishra's Bird & $\begin{array}{l}-10 \leq x_{1} \leq 0 \\
-6.5 \leq x_{2} \leq 0\end{array}$ & 2 & $\begin{array}{l}f(x)=-\sin \left(x_{2}\right) e^{\left[\left(1-\cos x_{1}\right)^{2}\right]}+\cos \left(x_{1}\right) e^{\left[\left(1-\sin x_{2}\right)^{2}\right]}+\left(x_{1}-x_{2}\right)^{2} \\
\text { subjectedto }:\left(x_{1}+5\right)^{2}+\left(x_{2}+5\right)^{2}<25\end{array}$ & -106.7645 \\
\hline 12 & Simionescu & $\begin{array}{l}-1.25 \leq x_{1} \leq 1.25 \\
-1.25 \leq x_{2} \leq 1.25\end{array}$ & 2 & $\begin{array}{l}f(x)=0.1 x_{1} x_{2}, \\
\text { subjectedto }: x_{1}^{2}+x_{2}^{2} \leq\left[1+0.2 \cos \left(8 \arctan \frac{x}{y}\right)\right]^{2}\end{array}$ & -0.0726 \\
\hline
\end{tabular}

Table 3 Performance parameters of different algorithms of benchmark equations without constraints

\begin{tabular}{|c|c|c|c|c|c|c|c|}
\hline Functions & parameters & $\mathrm{DE}$ & PSO & TLBO & SSA & CSA & PGCSA \\
\hline \multirow[t]{3}{*}{$\mathrm{F}_{1}$} & $\mathrm{BV}$ & $1.3757 \mathrm{e}-07$ & $7.0633 \mathrm{e}-06$ & $2.8895 e-14$ & $5.1238 \mathrm{e}-18$ & $2.9758 \mathrm{e}-18$ & $\mathbf{0}$ \\
\hline & AVG & $3.5016 \mathrm{e}-05$ & 0.0934 & 0.1778 & $9.4064 \mathrm{e}-14$ & $2.9649 \mathrm{e}-15$ & $8.4282 \mathrm{e}-16$ \\
\hline & SD & $6.0684 \mathrm{e}-05$ & 0.2302 & 0.3278 & $1.3123 \mathrm{e}-12$ & $3.3658 \mathrm{e}-16$ & $3.3311 \mathrm{e}-16$ \\
\hline \multirow[t]{3}{*}{$\mathrm{F}_{2}$} & $\mathrm{BV}$ & -1.0316 & -1.0316 & -1.0316 & -1.0316 & -1.0316 & -1.0316 \\
\hline & AVG & -1.0313 & -0.8850 & -1.0316 & -1.0316 & -1.0316 & -1.0316 \\
\hline & SD & $9.0355 e-04$ & 0.2956 & $2.9538 \mathrm{e}-13$ & $7.6704 \mathrm{e}-12$ & $1.2340 \mathrm{e}-04$ & $1.2443 \mathrm{e}-13$ \\
\hline \multirow[t]{3}{*}{$\mathrm{F}_{3}$} & $\mathrm{BV}$ & 0.0079 & 0.0018 & $2.9330 \mathrm{e}-09$ & $1.5438 \mathrm{e}-12$ & $1.7241 \mathrm{e}-07$ & $1.8657 \mathrm{e}-216$ \\
\hline & AVG & 2.4313 & 0.0035 & $3.9057 \mathrm{e}-08$ & $2.9919 \mathrm{e}-12$ & $4.2450 \mathrm{e}-06$ & $4.9540 \mathrm{e}-188$ \\
\hline & SD & 4.5129 & $9.6023 \mathrm{e}-04$ & $7.5544 \mathrm{e}-08$ & $9.5377 \mathrm{e}-13$ & $4.7588 \mathrm{e}-06$ & $\mathbf{0}$ \\
\hline \multirow[t]{3}{*}{$\mathrm{F}_{4}$} & $\mathrm{BV}$ & 0.6220 & 0.0057 & $4.7529 \mathrm{e}-04$ & $1.0830 \mathrm{e}-11$ & $6.8693 \mathrm{e}-09$ & $1.8797 \mathrm{e}-31$ \\
\hline & AVG & 1.6084 & 0.0085 & 0.0026 & $1.6452 \mathrm{e}-11$ & $3.7298 \mathrm{e}-08$ & $9.1358 \mathrm{e}-27$ \\
\hline & SD & 0.5904 & 0.0012 & 0.0015 & $3.4048 \mathrm{e}-12$ & $2.0570 \mathrm{e}-08$ & $2.5018 \mathrm{e}-26$ \\
\hline \multirow[t]{3}{*}{$\mathrm{F}_{5}$} & $\mathrm{BV}$ & $9.1213 e-04$ & $2.3457 \mathrm{e}-04$ & $3.3722 \mathrm{e}-04$ & $2.8919 \mathrm{e}-09$ & $2.1687 \mathrm{e}-06$ & $\mathbf{0}$ \\
\hline & $\mathrm{AVG}$ & 0.0024 & $3.1331 \mathrm{e}-04$ & 0.0025 & $5.3224 \mathrm{e}-09$ & $9.0129 \mathrm{e}-06$ & $\mathbf{0}$ \\
\hline & SD & 0.0012 & $5.6029 \mathrm{e}-05$ & 0.0013 & $1.3577 \mathrm{e}-09$ & $4.8453 \mathrm{e}-06$ & $\mathbf{0}$ \\
\hline \multirow[t]{3}{*}{$\mathrm{F}_{6}$} & $\mathrm{BV}$ & 0.2189 & $2.6564 \mathrm{e}-04$ & 0 & $1.0596 \mathrm{e}-10$ & $4.5475 \mathrm{e}-13$ & $\mathbf{0}$ \\
\hline & AVG & 0.4824 & $4.1718 \mathrm{e}-04$ & 6.7214 & 11.5415 & $1.4893 \mathrm{e}-12$ & $\mathbf{0}$ \\
\hline & $\mathrm{SD}$ & 0.1552 & $7.3929 \mathrm{e}-05$ & 33.9646 & 12.3709 & $6.9976 \mathrm{e}-13$ & $\mathbf{0}$ \\
\hline \multirow[t]{3}{*}{$\mathrm{F}_{7}$} & $\mathrm{BV}$ & $8.7759 \mathrm{e}-04$ & 0.0012 & $2.4047 \mathrm{e}-13$ & $3.0030 \mathrm{e}-12$ & $9.7673 \mathrm{e}-06$ & $\mathbf{0}$ \\
\hline & AVG & 0.0023 & 0.0020 & $8.4576 \mathrm{e}-11$ & 0.0033 & 0.0074 & $\mathbf{0}$ \\
\hline & $\mathrm{SD}$ & $9.8201 \mathrm{e}-04$ & 0.0014 & $1.4353 \mathrm{e}-10$ & 0.0061 & 0.0080 & $\mathbf{0}$ \\
\hline \multirow[t]{3}{*}{$\mathrm{F}_{8}$} & $\mathrm{BV}$ & 0.0243 & 0.0053 & $3.5947 \mathrm{e}-07$ & $1.7137 \mathrm{e}-05$ & $4.6370 \mathrm{e}-06$ & $8.8818 \mathrm{e}-16$ \\
\hline & AVG & 0.0381 & 0.0066 & $1.4767 \mathrm{e}-06$ & 1.3225 & 1.3240 & $2.2145 \mathrm{e}-14$ \\
\hline & $\mathrm{SD}$ & 0.0060 & $5.8767 \mathrm{e}-04$ & $1.1295 \mathrm{e}-06$ & 0.9674 & 0.8112 & $1.2478 \mathrm{e}-12$ \\
\hline \multirow[t]{3}{*}{$\mathrm{F}_{9}$} & $\mathrm{BV}$ & -959.6407 & -956.8922 & -959.6406 & -955.0593 & -959.6407 & -959.6407 \\
\hline & AVG & -948.0369 & -893.3701 & -954.5991 & -926.6889 & -950.2151 & -958.5351 \\
\hline & SD & 25.4528 & 55.0759 & 17.4195 & 30.8477 & 24.4413 & 2.4221 \\
\hline
\end{tabular}

Bold values indicate the best value 
Table 4 Performance parameters of different algorithms of benchmark equations with constraints

\begin{tabular}{llllllll}
\hline Functions & parameters & DE & PSO & TLBO & SSA & CSA & PGCSA \\
\hline $\mathrm{F}_{10}$ & BV & $1.0531 \mathrm{e}-05$ & $8.0940 \mathrm{e}-08$ & $1.0686 \mathrm{e}-07$ & $4.9911 \mathrm{e}-19$ & $8.3323 \mathrm{e}-30$ & $\mathbf{0}$ \\
& AVG & $2.0621 \mathrm{e}-04$ & $2.6692 \mathrm{e}-04$ & $2.6219 \mathrm{e}-06$ & $5.8949 \mathrm{e}-16$ & $2.3658 \mathrm{e}-27$ & $\mathbf{0}$ \\
& SD & $3.3081 \mathrm{e}-04$ & 0.0014 & $3.7181 \mathrm{e}-06$ & $1.3688 \mathrm{e}-15$ & $2.8840 \mathrm{e}-27$ & $\mathbf{0}$ \\
$\mathrm{F}_{11}$ & BV & -106.7645 & -106.7645 & -106.7645 & -106.7645 & -106.7645 & $-\mathbf{1 0 6 . 7 6 4 5}$ \\
& AVG & -106.7638 & -106.7641 & -106.7619 & -106.7645 & -106.7645 & $-\mathbf{1 0 6 . 7 6 4 5}$ \\
& SD & 0.0013 & $4.4961 \mathrm{e}-04$ & 0.0041 & $2.0587 \mathrm{e}-07$ & $1.9211 \mathrm{e}-12$ & $\mathbf{2 . 9 5 0 4 e - 1 4}$ \\
$\mathrm{F}_{12}$ & BV & -0.0720 & -0.0720 & -0.0720 & -0.0720 & -0.0720 & $-\mathbf{0 . 0 7 2 0}$ \\
& AVG & -0.0716 & -0.0717 & -0.0716 & -0.0720 & -0.0720 & $-\mathbf{0 . 0 7 2 0}$ \\
& SD & $2.9700 \mathrm{e}-04$ & $5.4975 \mathrm{e}-04$ & $3.5832 \mathrm{e}-04$ & $1.9955 \mathrm{e}-05$ & $4.5950 \mathrm{e}-11$ & $\mathbf{3 . 9 0 8 3 e}-\mathbf{1 7}$ \\
\hline
\end{tabular}

Bold values indicate the best value

supplemental prevention like changing the concealing places to stay away from becoming an upcoming easy target. By considering these clever action CSA algorithm has developed. The ideas of CSA are check listed as given below:

i. Crows reside like a group.

ii. Crows remember the location of their concealing places of food.

iii. Crows observe and follow one another to rustle food.

Like other optimization techniques initialization of CSA is quite similar. In initialization phase, the flock of crows is initialized randomly by conceding designed variables (D) and number of crows (NC) by satisfying the constraints as characterized in Eq. (5). Each row of the matrix represents one crow in the flock and each column represents one design variable of the problem. Each crow denotes a feasible solution of the problem.

Crows $=\left[\begin{array}{cccc}X_{1}^{1} & X_{2}^{1} & \cdots & X_{D}^{1} \\ X_{1}^{2} & X_{2}^{2} & \cdots & X_{D}^{2} \\ \vdots & \vdots & \vdots & \vdots \\ X_{1}^{N_{C}} & X_{2}^{N_{C}} & \cdots & X_{D}^{N_{C}}\end{array}\right]$

$\mathrm{X}$ represents the design variable of a crow. In the first iteration, assume that they have concealed their foods in the initial position because crows have less experience in the beginning. The memory (M) of each crow is initialized as described in Eq. (6).

Memory $=\left[\begin{array}{cccc}M_{1}^{1} & M_{2}^{1} & \cdots & M_{D}^{1} \\ M_{1}^{2} & M_{2}^{2} & \cdots & M_{D}^{2} \\ \vdots & \vdots & \vdots & \vdots \\ M_{1}^{N_{C}} & M_{2}^{N_{C}} & \cdots & M_{D}^{N_{C}}\end{array}\right]$

The memory matrix represents the best feasible solutions of crows obtained so far. $\mathrm{M}$ is the element of memory matrix which represents the design variable of a memory of crow. The fitness value can be calculated by putting the value of designed variables (D) in the objective function. The crows update their position with the help of other crow. The ith crow finds their food by following and observing another jth crow. The jth crow tries to fool the ith crow by changing the location of the food on the territory by knowing the intention of opponent crow. The updated position of the crow is characterized in Eq. (7).

$X_{\text {new }}^{i}=\left\{\begin{array}{cc}X_{\text {old }}^{i}+r \times f l^{i} \times\left(M^{j}-X_{\text {old }}^{i}\right) & r_{1} \geq A P \\ L B+(U B-L B) \times \text { rand } & \text { Otherwise }\end{array}\right.$

where, ' $r$ ' and ' $r_{1}$ ' are two random numbers within the range from 0 to 1 . 'AP' is the awareness probability of crow. Small value of AP enhances the intensification and the high value of AP enhances the diversification. The memory of crow is updated with fitter crow position as depicted in Eq. (8).

$M_{\text {new }}^{i}= \begin{cases}X_{\text {new }}^{i} & f\left(X_{\text {new }}^{i}\right) \geq f\left(M_{\text {old }}^{i}\right) \\ M_{\text {old }}^{i} & \text { Otherwise }\end{cases}$

\subsection{Proposed PSO-based group oriented CSA (PGCSA)}

CSA is categorized into two phases (phase-1 and phase-2) by concerning the awareness probability (AP). In phase- 1 (without awareness probability), if the jth crow $\left(\mathrm{X}_{\mathrm{j}}\right)$ is unaware that $i^{\text {th }}$ crow $\left(X_{i}\right)$ is following it, i.e. $r_{1} \geq A P$, then the ith crow $\left(\mathrm{X}_{\mathrm{i}}\right)$ is supposed to follow the food hiding place of $j^{\text {th }}$ crow $\left(\mathrm{M}_{\mathrm{j}}\right)$ as characterized in (7). If the functional value of $X_{i}$ is better than the functional value of $\mathrm{M}_{\mathrm{j}}$, then the crow with better functional value will follow the worst one. This may downturn the convergence rate of the algorithm to achieve the optimal solution. In this work, the entire flock of crows is subdivided into small groups. The fittest crow of the corresponding group is considered as leader and the rest of the crows of that group (followers) 

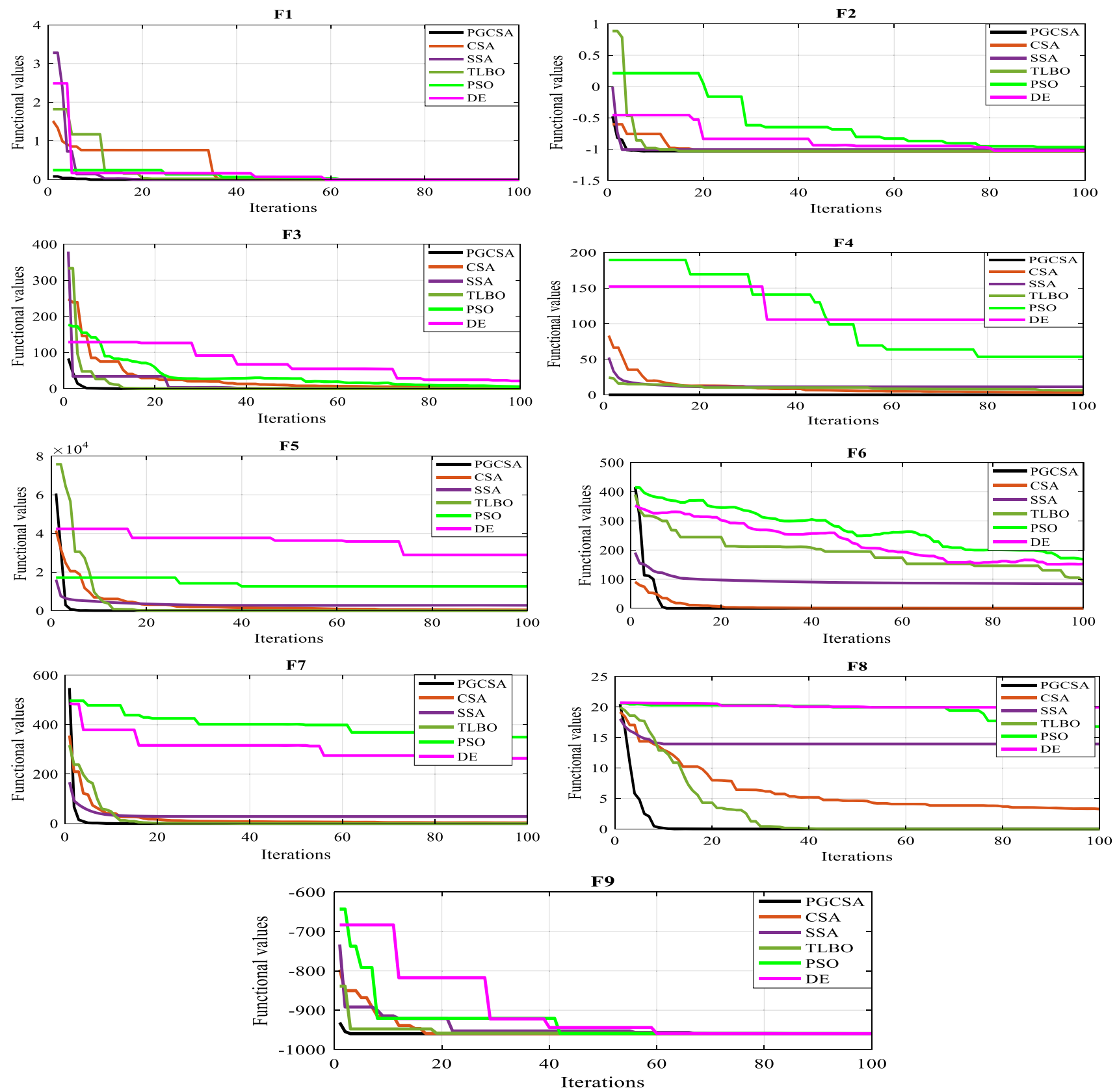

Fig. 4 Convergence plot of PGCSA, CSA, SSA, TLBO, PSO and DE on the benchmark functions without constraint

are updated by following the leader crow as characterized in (9).

$X_{\text {new }}^{i}=X_{\text {old }}^{i}+r \times f l^{i} \times\left(X_{L}^{k}-X_{\text {old }}^{i}\right)$, If $\quad r_{1} \geq A P$

$\mathrm{k}=1,2,3, \ldots, \mathrm{ng}$. Where, 'ng' is the number of groups. Crows are distributed among the groups by conceding their weight (W) as characterized in Eq. (10) [55].
$W^{i}=\exp \left(-D \frac{f\left(X^{i}\right)-f\left(X^{\text {Best }}\right)}{\sum_{i=1}^{N_{c}} f\left(X^{i}\right)-f\left(X^{B e s t}\right)}\right)$

Weight (W) is evaluated in such a manner that $\mathrm{W}$ of better functional value is higher. $\mathrm{W}$ of the best crow is one and for other crows are in between 0 to 1 . The crows with highest $\mathrm{W}$ are chosen as group leaders. The followers are distributed among the groups by conceding the weight of 

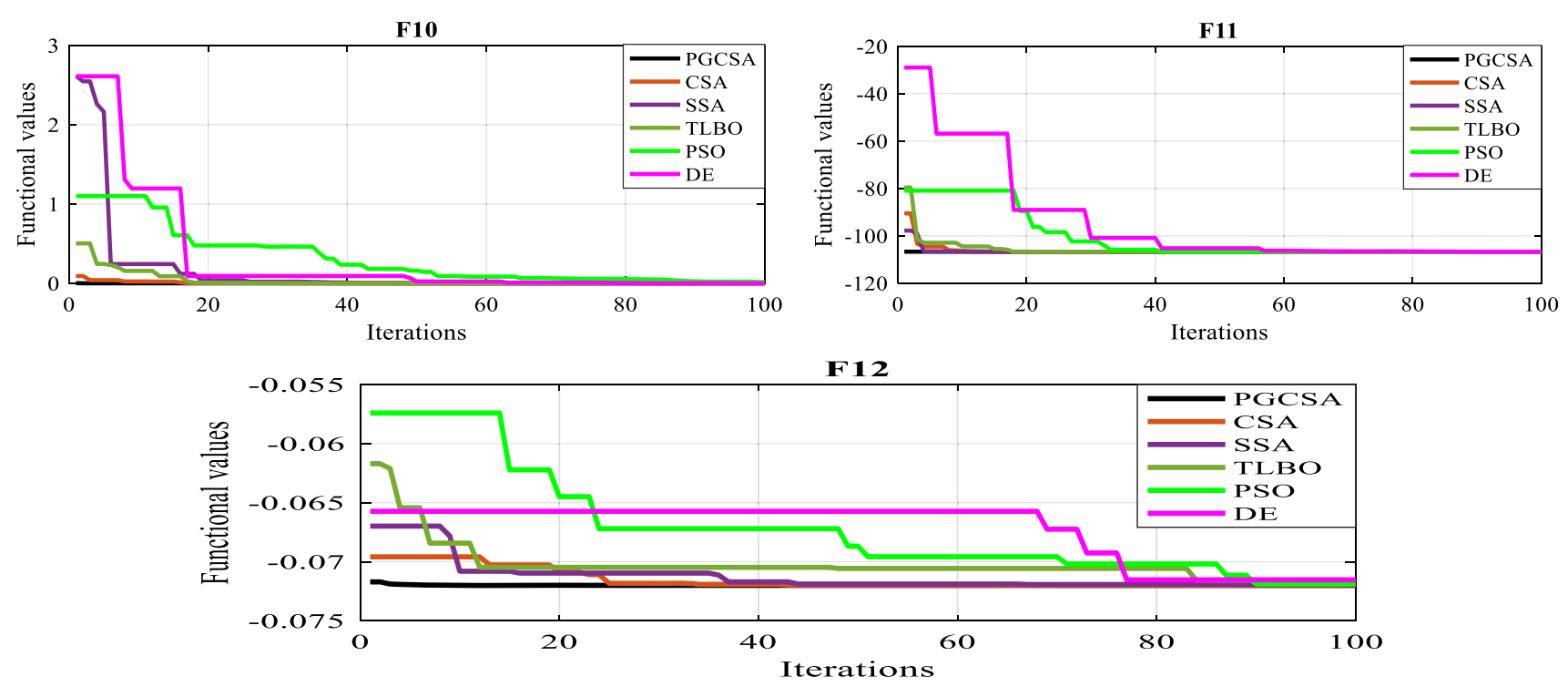

Fig. 5 Convergence plot of PGCSA, CSA, SSA, TLBO, PSO and DE on the benchmark functions with constraint

Table $5 p$-Values obtained from the Wilcoxon signed rank test

Table 6 Rank sum values obtained from the Wilcoxon signed rank test

\begin{tabular}{llllll}
\hline & PGCSA Vs. DE & PGCSA Vs. PSO & PGCSA Vs. TLBO & PGCSA Vs. SSA & PGCSA Vs. CSA \\
\hline $\mathrm{F}_{1}$ & $1.7344 \mathrm{E}-06$ & $1.7344 \mathrm{E}-06$ & $1.7344 \mathrm{E}-06$ & $1.7344 \mathrm{E}-06$ & $1.7344 \mathrm{E}-06$ \\
$\mathrm{~F}_{2}$ & $1.707 \mathrm{E}-06$ & $1.92092 \mathrm{E}-06$ & $1.70729 \mathrm{E}-06$ & 0.002873 & $1.7344 \mathrm{e}-06$ \\
$\mathrm{~F}_{3}$ & $1.7344 \mathrm{E}-06$ & $1.73222 \mathrm{E}-06$ & $1.7344 \mathrm{E}-06$ & $1.7344 \mathrm{E}-06$ & $1.7344 \mathrm{E}-06$ \\
$\mathrm{~F}_{4}$ & $1.7344 \mathrm{E}-06$ & $1.7344 \mathrm{E}-06$ & $1.7344 \mathrm{E}-06$ & $1.7344 \mathrm{E}-06$ & $1.7344 \mathrm{E}-06$ \\
$\mathrm{~F}_{5}$ & $1.7344 \mathrm{E}-06$ & $1.7344 \mathrm{E}-06$ & $1.7344 \mathrm{E}-06$ & $1.7344 \mathrm{E}-06$ & $1.7344 \mathrm{E}-06$ \\
$\mathrm{~F}_{6}$ & $1.7344 \mathrm{E}-06$ & $1.7344 \mathrm{E}-06$ & 0.0165 & $1.7344 \mathrm{E}-06$ & $1.72351 \mathrm{E}-06$ \\
$\mathrm{~F}_{7}$ & $1.7344 \mathrm{E}-06$ & $1.7344 \mathrm{E}-06$ & $1.7344 \mathrm{E}-06$ & $1.7344 \mathrm{E}-06$ & $1.72351 \mathrm{E}-06$ \\
$\mathrm{~F}_{8}$ & $1.7344 \mathrm{E}-06$ & $1.7344 \mathrm{E}-06$ & $1.7344 \mathrm{E}-06$ & $1.7344 \mathrm{E}-06$ & $1.72351 \mathrm{E}-06$ \\
$\mathrm{~F}_{9}$ & 0.040475473 & $1.97295 \mathrm{E}-05$ & 0.011748106 & 0.006809395 & 0.0050 \\
$\mathrm{~F}_{10}$ & $1.7344 \mathrm{E}-06$ & $1.7344 \mathrm{E}-06$ & $1.7344 \mathrm{E}-06$ & $1.7344 \mathrm{E}-06$ & $1.7344 \mathrm{E}-06$ \\
$\mathrm{~F}_{11}$ & $1.7344 \mathrm{E}-06$ & $1.7344 \mathrm{E}-06$ & $1.7344 \mathrm{E}-06$ & $1.7344 \mathrm{E}-06$ & NA \\
$\mathrm{F}_{12}$ & $1.7344 \mathrm{E}-06$ & $1.7344 \mathrm{E}-06$ & $1.7344 \mathrm{E}-06$ & $1.7344 \mathrm{E}-06$ & $1.7344 \mathrm{E}-06$ \\
\hline
\end{tabular}

\begin{tabular}{llllllllllllll}
\hline Test Functions & & $\mathrm{F}_{1}$ & $\mathrm{~F}_{2}$ & $\mathrm{~F}_{3}$ & $\mathrm{~F}_{4}$ & $\mathrm{~F}_{5}$ & $\mathrm{~F}_{6}$ & $\mathrm{~F}_{7}$ & $\mathrm{~F}_{8}$ & $\mathrm{~F}_{9}$ & $\mathrm{~F}_{10}$ & $\mathrm{~F}_{11}$ & $\mathrm{~F}_{12}$ \\
\hline PGCSA Vs. CSA & $\mathrm{R}^{+}$ & $\mathbf{4 6 5}$ & $\mathbf{4 6 5}$ & $\mathbf{4 6 5}$ & $\mathbf{4 6 5}$ & $\mathbf{4 6 5}$ & $\mathbf{4 6 5}$ & $\mathbf{4 6 5}$ & $\mathbf{4 6 5}$ & $\mathbf{5 5}$ & $\mathbf{4 6 5}$ & $\mathrm{NA}$ & $\mathbf{4 6 5}$ \\
& $\mathrm{R}^{-}$ & 0 & 0 & 0 & 0 & 0 & 0 & 0 & 0 & 0 & 0 & $\mathrm{NA}$ & 0 \\
PGCSA Vs. SSA & $\mathrm{R}^{+}$ & $\mathbf{4 6 5}$ & $\mathbf{1 0 9}$ & $\mathbf{4 6 5}$ & $\mathbf{4 6 5}$ & $\mathbf{4 6 5}$ & $\mathbf{4 6 5}$ & $\mathbf{4 6 5}$ & $\mathbf{4 6 5}$ & $\mathbf{3 6 4}$ & $\mathbf{4 6 5}$ & $\mathbf{4 6 5}$ & $\mathbf{4 6 5}$ \\
& $\mathrm{R}^{-}$ & 0 & 44 & 0 & 0 & 0 & 0 & 0 & 0 & 101 & 0 & 0 & 0 \\
PGCSA Vs. TLBO & $\mathrm{R}^{+}$ & $\mathbf{4 6 5}$ & $\mathbf{4 6 5}$ & $\mathbf{4 6 5}$ & $\mathbf{4 6 5}$ & $\mathbf{4 6 5}$ & $\mathbf{6 3}$ & $\mathbf{4 6 5}$ & $\mathbf{4 6 5}$ & $\mathbf{3 5 5}$ & $\mathbf{4 6 5}$ & $\mathbf{4 6 5}$ & $\mathbf{4 6 5}$ \\
& $\mathrm{R}^{-}$ & 0 & 0 & 0 & 0 & 0 & 14 & 0 & 0 & 110 & 0 & 0 & 0 \\
PGCSA Vs. PSO & $\mathrm{R}^{+}$ & $\mathbf{4 6 5}$ & $\mathbf{4 6 4}$ & $\mathbf{4 6 5}$ & $\mathbf{4 6 5}$ & $\mathbf{4 6 5}$ & $\mathbf{4 6 5}$ & $\mathbf{4 6 5}$ & $\mathbf{4 6 5}$ & $\mathbf{4 4 0}$ & $\mathbf{4 6 5}$ & $\mathbf{4 6 5}$ & $\mathbf{4 6 5}$ \\
& $\mathrm{R}^{-}$ & 0 & 1 & 0 & 0 & 0 & 0 & 0 & 0 & 25 & 0 & 0 & 0 \\
PGCSA Vs. DE & $\mathrm{R}^{+}$ & $\mathbf{4 6 5}$ & $\mathbf{4 6 5}$ & $\mathbf{4 6 5}$ & $\mathbf{4 6 5}$ & $\mathbf{4 6 5}$ & $\mathbf{4 6 5}$ & $\mathbf{4 6 5}$ & $\mathbf{4 6 5}$ & $\mathbf{3 2 5}$ & $\mathbf{4 6 5}$ & $\mathbf{4 6 5}$ & $\mathbf{4 6 5}$ \\
& $\mathrm{R}^{-}$ & 0 & 0 & 0 & 0 & 0 & 0 & 0 & 0 & 140 & 0 & 0 & 0 \\
\hline
\end{tabular}

Bold values indicate the best value 
Table 7 Comparison of performance measures of testing period of IXIC index

\begin{tabular}{llll}
\hline Prediction models & MAE & MSE & MAPE \\
\hline PGCSA-ELM & 16.39424 & 410.5365 & 0.9264 \\
CSA-ELM & 18.02204 & 534.7980 & 0.9984 \\
ELM & 21.66214 & 708.8436 & 1.1807 \\
GARCH-DAN2 [10] & 109.626 & $20,901.198$ & \\
DAN2 [10] & 32.875 & 1472.278 & \\
GARCH-MLP [10] & 42.739 & 3665.8387 & \\
MLP [10] & 41.153 & 2478.1468 & \\
\hline
\end{tabular}

the crows. The numbers of crows in one group are evaluated by using Eqs. (11) and (12).

$\begin{aligned} \alpha^{i} & =\frac{W_{i}^{G}}{\sum_{i=1}^{n g} W_{i}^{G}} \\ N_{G_{i}} & =\operatorname{round}\left(\alpha^{i} \times N_{f}\right)\end{aligned}$
Table 8 Comparison of performance measures of testing period of GDAXI index

\begin{tabular}{llll}
\hline Prediction models & MAE & MSE & MAPE \\
\hline PGCSA-ELM & $\mathbf{1 1 4 . 6 4 4 8}$ & $\mathbf{4 1 , 2 6 9 . 5 2 5 6}$ & $\mathbf{1 . 8 2 6 4}$ \\
CSA-ELM & 167.1929 & $48,990.5315$ & 2.6483 \\
ELM & 207.0571 & $85,608.8853$ & 3.1201 \\
GA-NN [54] & & & 3.49 \\
GRNN [54] & & & 10.75 \\
RBE [54] & & & 12.3 \\
BNNMAS [54] & & & 2.84 \\
\hline
\end{tabular}

Bold values indicate the best value

where, $\mathrm{W}_{\mathrm{i}}^{\mathrm{G}}, N_{G_{i}}$, and $\mathrm{N}_{\mathrm{f}}$ are weight of the group leader, numbers of group members, and numbers of followers respectively. By this approach, the local search space is enhanced and explored. In phase-2 (with awareness probability), if $X_{j}$ is aware that $X_{i}$ is following it, i.e. $r_{1}<A P$, then the ith crow $\left(\mathrm{X}_{\mathrm{i}}\right)$ is replaced by a random position as characterized in (7). $X_{i}$ may have better functional value than the random position of crow.

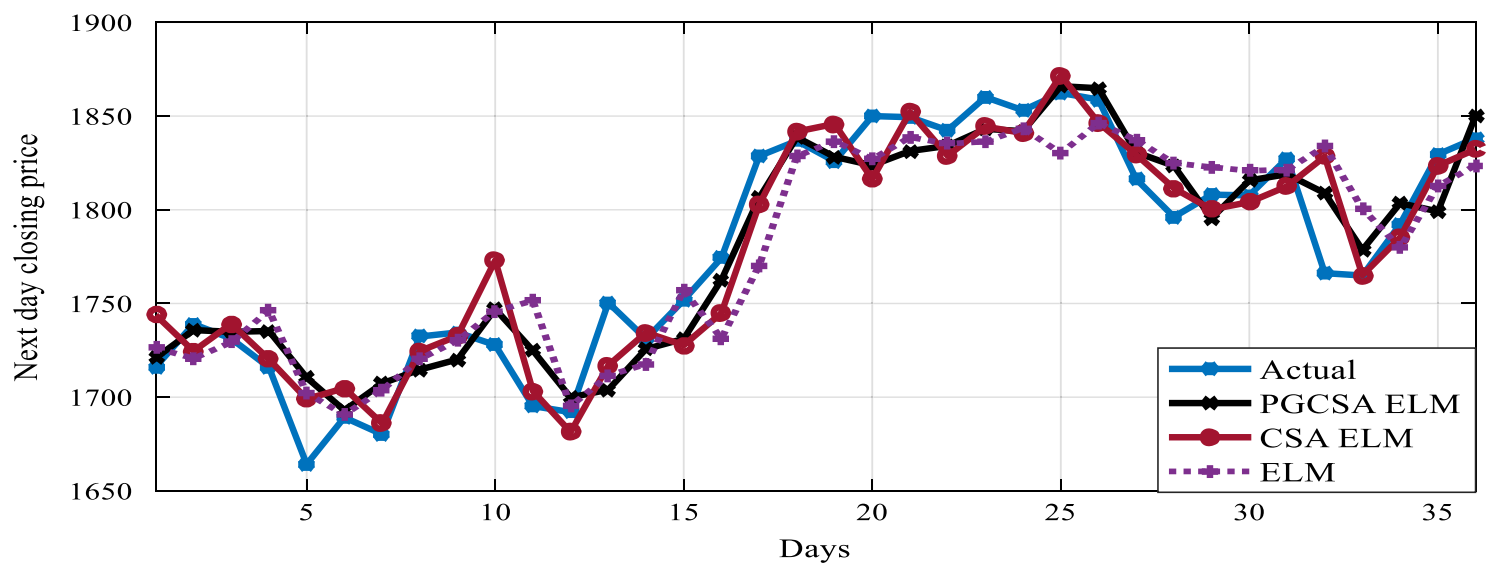

(a)

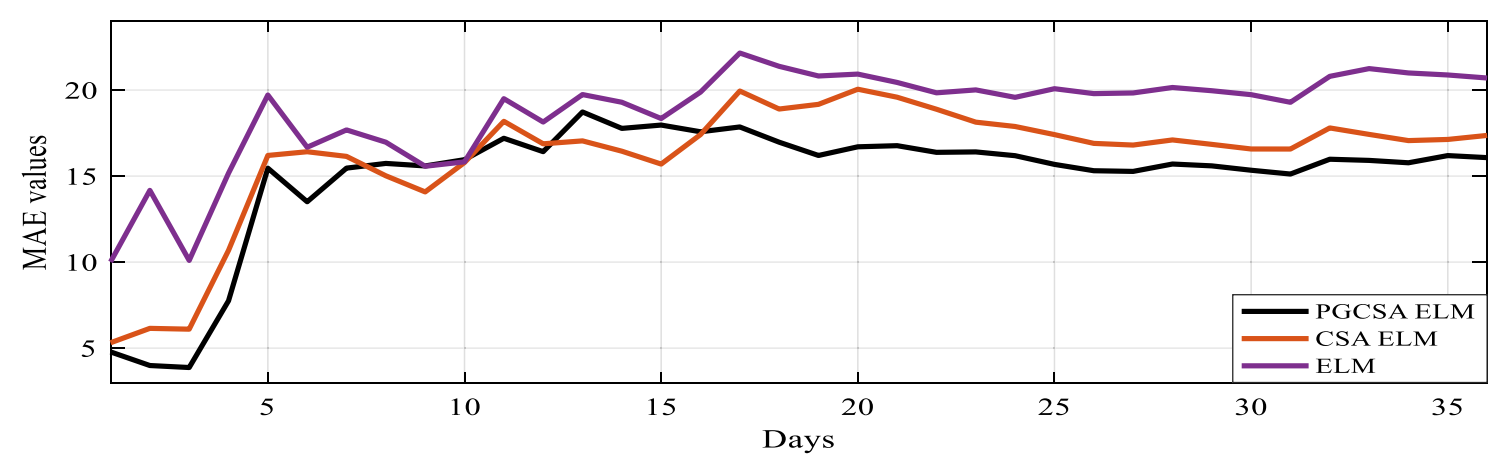

(b)

Fig. 6 a Next day predicted and actual closing price of IXIC index, b MAE values 


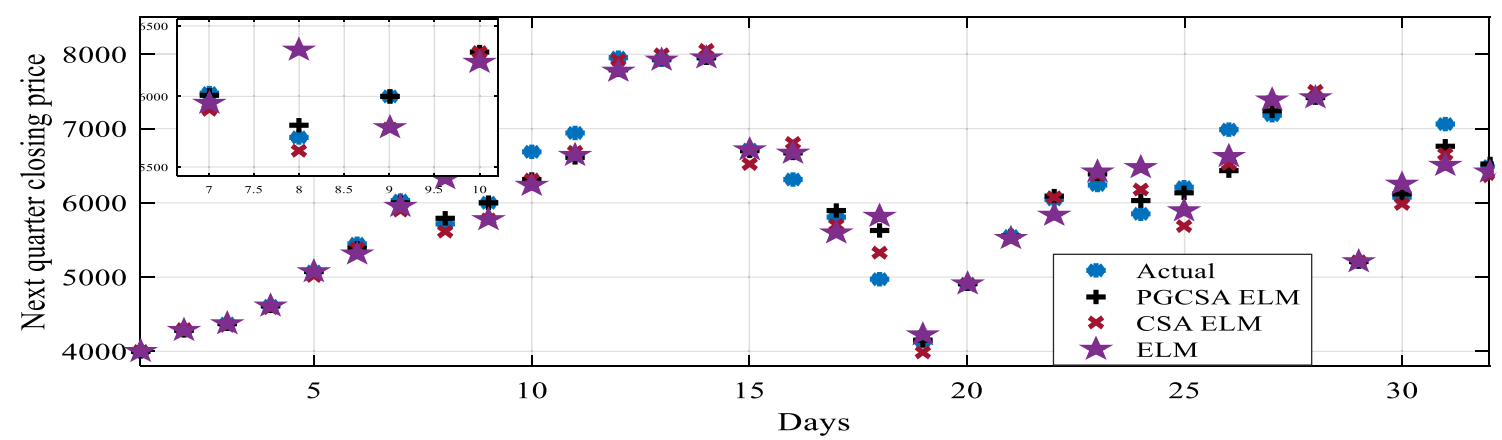

(a)

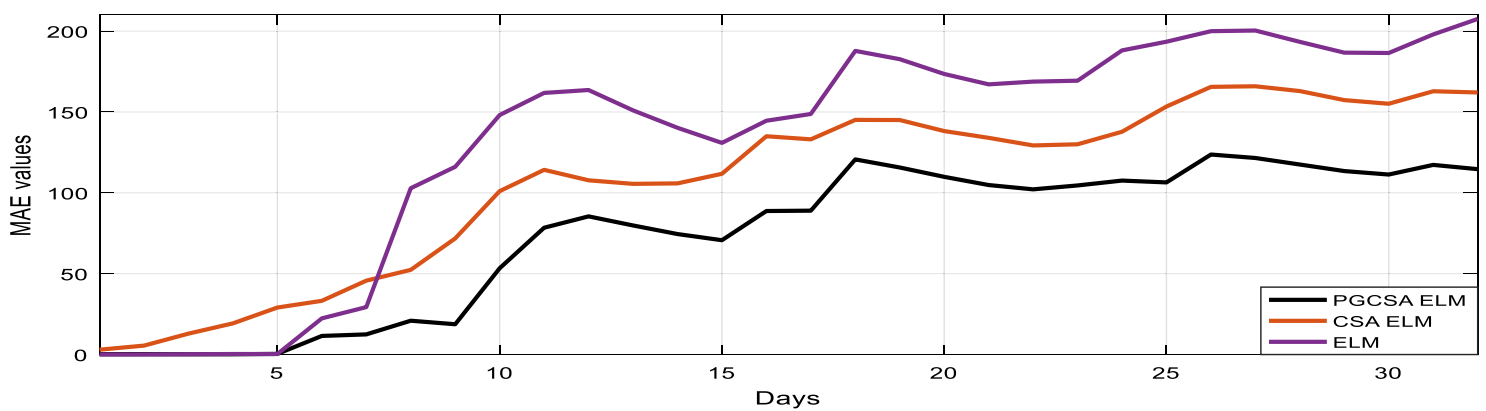

(b)

Fig. 7 a Next quarter predicted and actual closing price of GDAXI index, b MAE values

Table 9 Statistical analysis of ELM models with different neurons for IXIC market

\begin{tabular}{llll}
\hline Number of neurons & MSE & MAE & MAPE \\
\hline 5 & 9235.6564 & 145.2354 & 2.8546 \\
6 & 8123.5545 & 98.2454 & 1.8978 \\
7 & 7849.0144 & 89.4578 & 1.6112 \\
8 & 7852.8978 & 88.4574 & 1.5467 \\
9 & 7596.2542 & 75.2154 & 1.3745 \\
$\mathbf{1 0}$ & $\mathbf{7 5 1 6 . 9 9 7 2}$ & $\mathbf{6 1 . 0 2 4 8}$ & $\mathbf{0 . 9 7 8 7}$ \\
11 & 7529.2145 & 66.2454 & 0.9987 \\
12 & 7654.5215 & 68.2454 & 1.1568 \\
13 & 7894.8754 & 72.4542 & 1.3217 \\
14 & 8017.2459 & 82.4754 & 1.6547 \\
15 & 8178.1476 & 92.2584 & 1.8645 \\
\hline
\end{tabular}

Bold values indicate the best value

Table 10 Statistical analysis of ELM models with different activation functions for IXIC market

\begin{tabular}{llll}
\hline Activation functions & MSE & MAE & MAPE \\
\hline Sigmoid & $\mathbf{7 5 1 6 . 9 9 7 2}$ & 61.0248 & $\mathbf{0 . 9 7 8 7}$ \\
Tanh & 7517.6245 & $\mathbf{6 0 . 4 9 8 7}$ & 0.9789 \\
Softsign & 7519.8455 & 64.5612 & 0.9975 \\
ReLU & 7517.0148 & 61.0197 & 0.9792
\end{tabular}

Bold values indicate the best value
Table 11 MSE, MAE and MAPE values of PGCSA-ELM, CSA-ELM and ELM models for various markets

\begin{tabular}{lllll}
\hline Markets & Prediction Model & MSE & MAE & MAPE \\
\hline DJI & PGCSA-ELM & $\mathbf{5 5 2 9 . 6 9 2 9}$ & $\mathbf{4 3 . 7 4 8 1}$ & $\mathbf{0 . 1 9 5 4}$ \\
& CSA-ELM & $18,933.3649$ & 98.3678 & 0.4334 \\
& ELM & $53,801.1195$ & 156.7445 & 0.6625 \\
HIS & PGCSA-ELM & $\mathbf{6 2 3 0 . 7 5 6 7}$ & $\mathbf{4 7 . 8 3 9 8}$ & $\mathbf{0 . 1 8 7 1}$ \\
& CSA-ELM & $23,388.3681$ & 98.5441 & 0.3878 \\
& ELM & $67,974.8043$ & 187.5332 & 0.7133 \\
IXIC & PGCSA-ELM & $\mathbf{1 1 1 3 . 2 8 6 9}$ & $\mathbf{1 7 . 6 4 5 8}$ & $\mathbf{0 . 2 6 2 4}$ \\
& CSA-ELM & 1442.9564 & 26.9485 & 0.3942 \\
& ELM & 7516.9972 & 61.0248 & 0.9787 \\
N100 & PGCSA-ELM & $\mathbf{7 . 9 8 5 3}$ & $\mathbf{1 . 7 0 4 0}$ & $\mathbf{0 . 1 7 7 9}$ \\
& CSA-ELM & 32.0015 & 3.7812 & 0.3872 \\
& ELM & 80.0999 & 6.1061 & 0.6272 \\
\multirow{2}{*}{ NSEI } & PGCSA-ELM & $\mathbf{6 4 2 . 7 7 8 1}$ & $\mathbf{1 6 . 3 2 4 8}$ & $\mathbf{0 . 1 7 0 9}$ \\
& CSA-ELM & 4623.7704 & 46.0476 & 0.4599 \\
& ELM & $12,856.7450$ & 85.9799 & 0.8878 \\
\multirow{2}{*}{ RUT } & PGCSA-ELM & $\mathbf{2 4 . 9 1 4 3}$ & $\mathbf{3 . 6 6 0 3}$ & $\mathbf{0 . 2 7 0 2}$ \\
& CSA-ELM & 192.0929 & 9.5494 & 0.6751 \\
& ELM & 319.8097 & 13.4640 & 0.9419 \\
\multirow{2}{*}{ GDAXI } & PGCSA-ELM & $\mathbf{8 3 7 . 8 7 3 7}$ & $\mathbf{9 6 . 0 9 7 3}$ & $\mathbf{0 . 1 7 0 2}$ \\
& CSA-ELM & 5636.0005 & 101.4636 & 0.4452 \\
& ELM & $33,374.1955$ & 131.4623 & 1.1595 \\
\hline B & & &
\end{tabular}

Bold values indicate the best value 
Algorithm 1. Pseudo code of PGCSA algorithm.

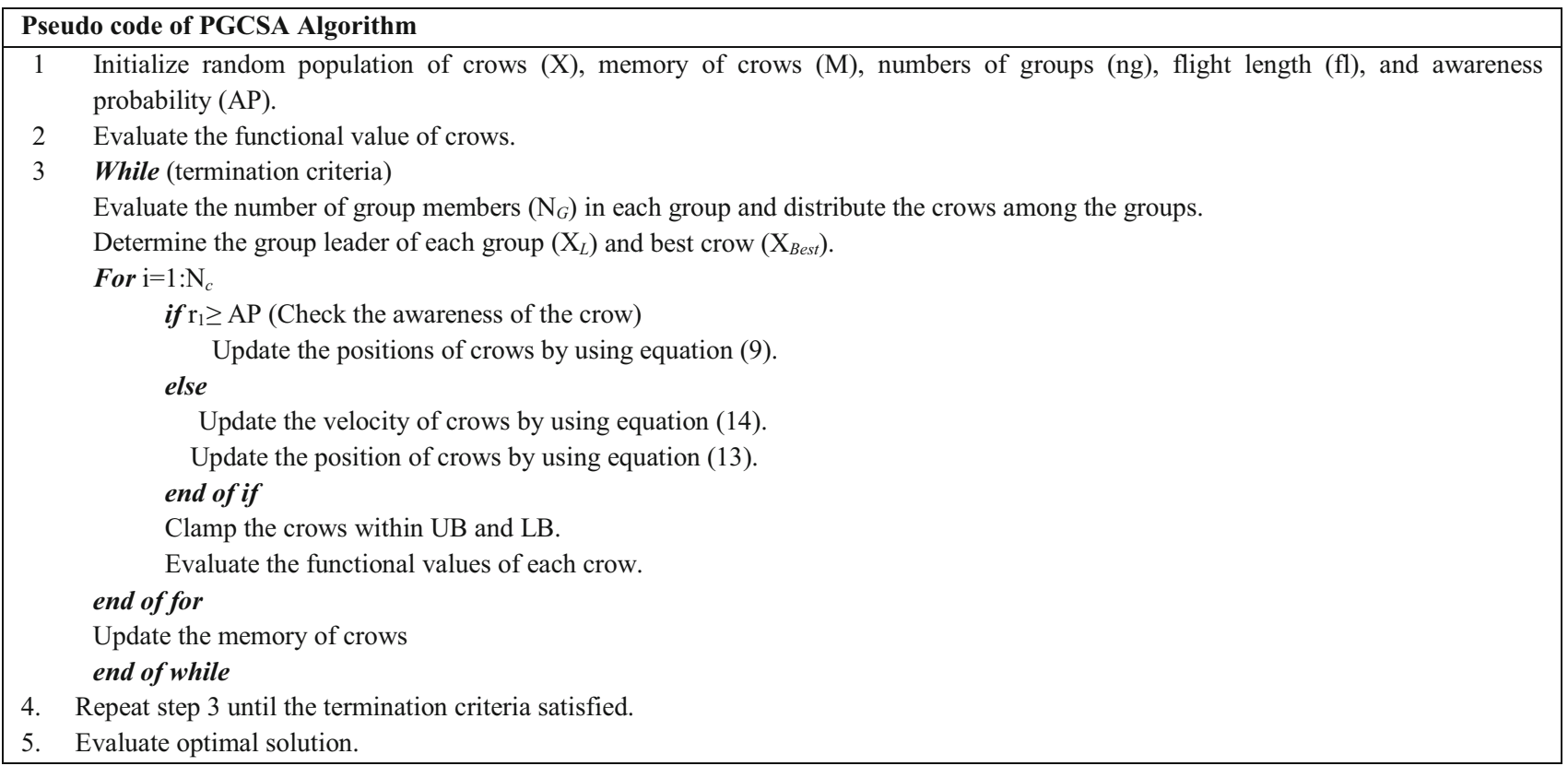

In the later stage of iteration, there is a higher probability that fittest $X_{i}$ may be replaced by random position. In this work, the second stage of Eq. (7) is modified by conceding the velocity as depicted in (13).

$X_{\text {new }}^{i}=X_{\text {old }}^{i}+v^{i}$

where, $v^{i}$ is the velocity of ith particle evaluated in the same fashion as in PSO [44]. Velocity (v) is updated by conceding the memory $(\mathrm{M})$ and best crow as depicted in (14).

$$
\begin{aligned}
v_{\text {new }}^{i}= & w \times v_{\text {old }}^{i}+\text { rand } \times c_{1} \times\left(M^{i}-X^{i}\right)+\text { rand } \times c_{2} \\
& \times\left(X^{\text {Best }}-X^{i}\right)
\end{aligned}
$$

Weightw $=0.9-\left[0.5 \frac{i t}{\text { itermax }}\right]$

where, $c_{1}$ and $c_{2}$ are the participation factors of $M$ and $X_{\text {Best }}$ respectively. $\mathrm{M}$ is memory of crows. This approach is used to enhance the exploitation. The movement of crows is illustrated in Fig. 2. The flow chart of proposed PGCSA algorithm is depicted in Fig. 3. The algorithm is briefly elaborated through pseudo code in algorithm 1 . The main benefits of this proposed PGCSA algorithm are as follows:

i. In first phase (without awareness probability), the subdivided groups of crows throughout the search space will help to explore local optima. So, it enhances the exploration capability of the algorithm.

ii. In second phase (with awareness probability), the velocity concept of PSO algorithm enhances the exploitation capability by contributing a direction towards optimal point. This approach helps to avoid the solution trapped into local optima.

iii. PGCSA algorithm enhances the balance between exploration and exploitation capability of the technique. The capability to solve high dimensional problem is enhanced with these two approaches.

\subsection{Research data}

In this work, the time series historical price of period from 1st January 2004 to 10th May 2020 of seven stock indices such as Dow Jones Industrial Average (DJI), Hang Seng Index (HSI), Nasdaq Composite (IXIC), Euronext-100 (N 100), Nifty 50 (NSEI), Russell 2000 (RUT) and DAX performance index (GDAXI) are considered for SM forecasting collected from (https://www.investing.com). Daily high, low and open prices of these indices are treated as inputs of ELM to predict the closing price of next day. The normalized data of open, low, high and closing prices are determined by employing Eq. (15).

$S=\frac{x-x_{\min }}{x_{\max }-x_{\min }}$

The training and testing data are considered in ratio of $7: 3$, respectively. The actual closing price is determined by de-normalizing the output of ELM as formulated in (16).

$X=S *\left(x_{\max }-x_{\min }\right)+x_{\min }$

where, $\mathrm{S}, x_{\max }$ and $x_{\min }$ are the normalized data, maximum value and minimum value, respectively. 

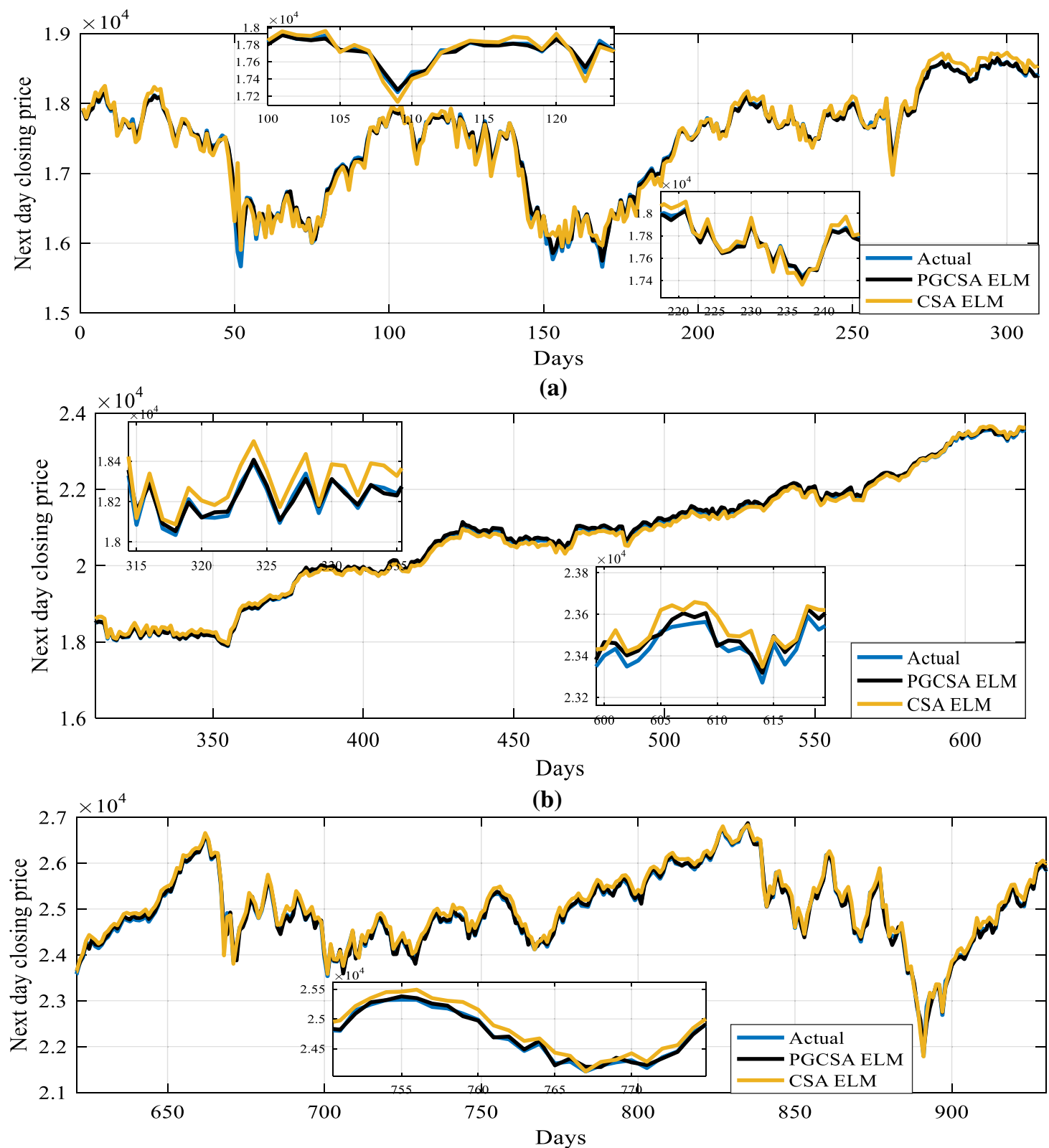

(c)

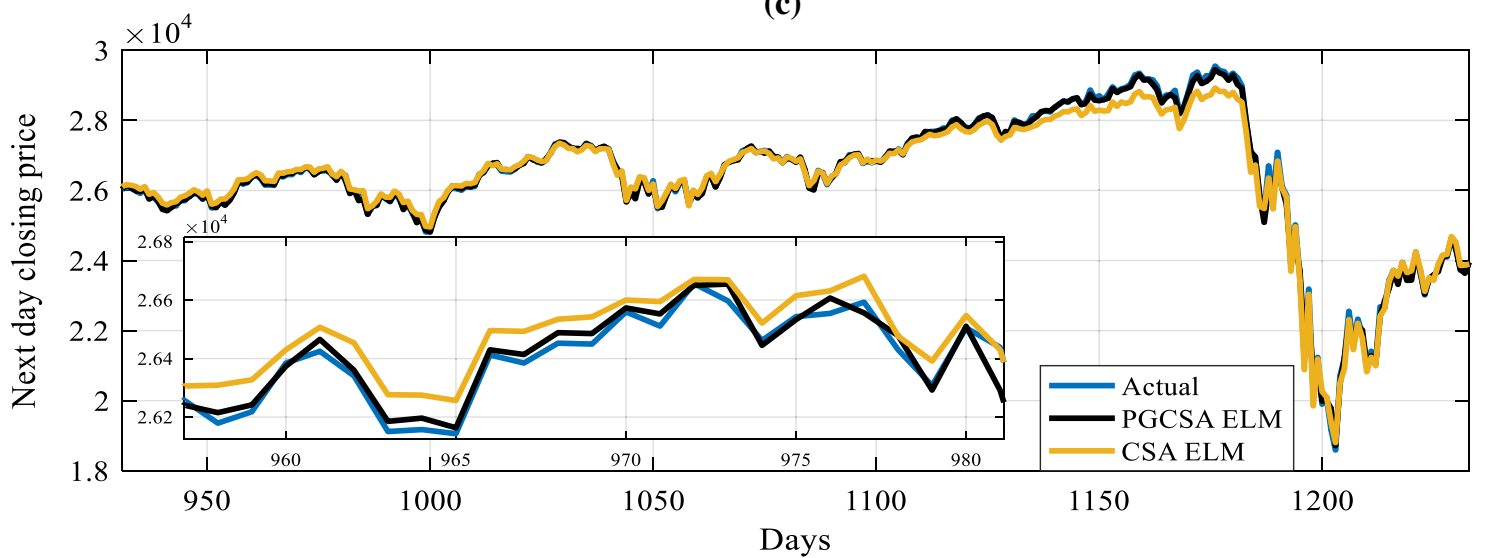

(d)

Fig. 8 PGCSA ELM and CSA ELM models predicted next day closing price of DJI 


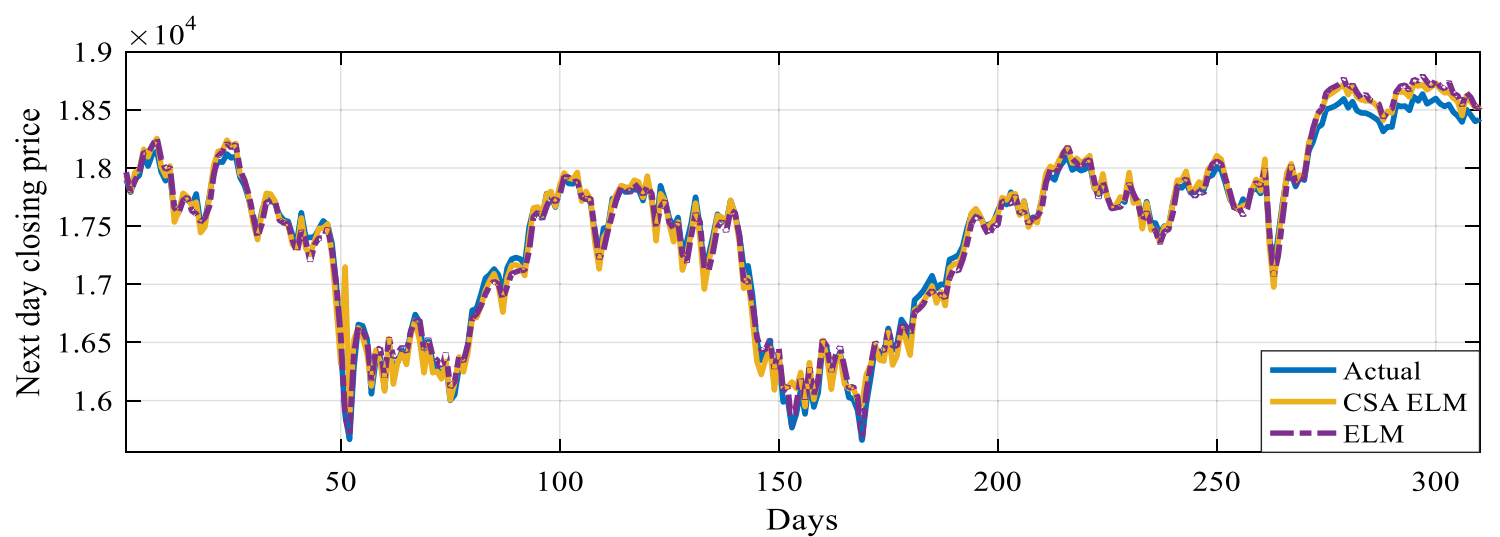

(a)

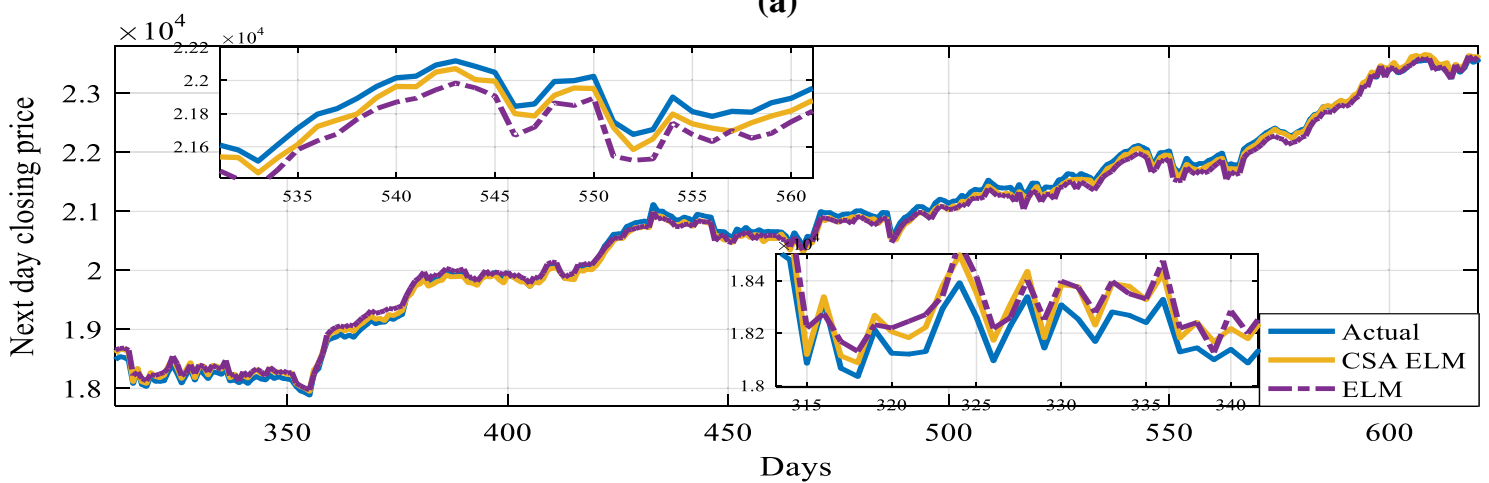

(b)

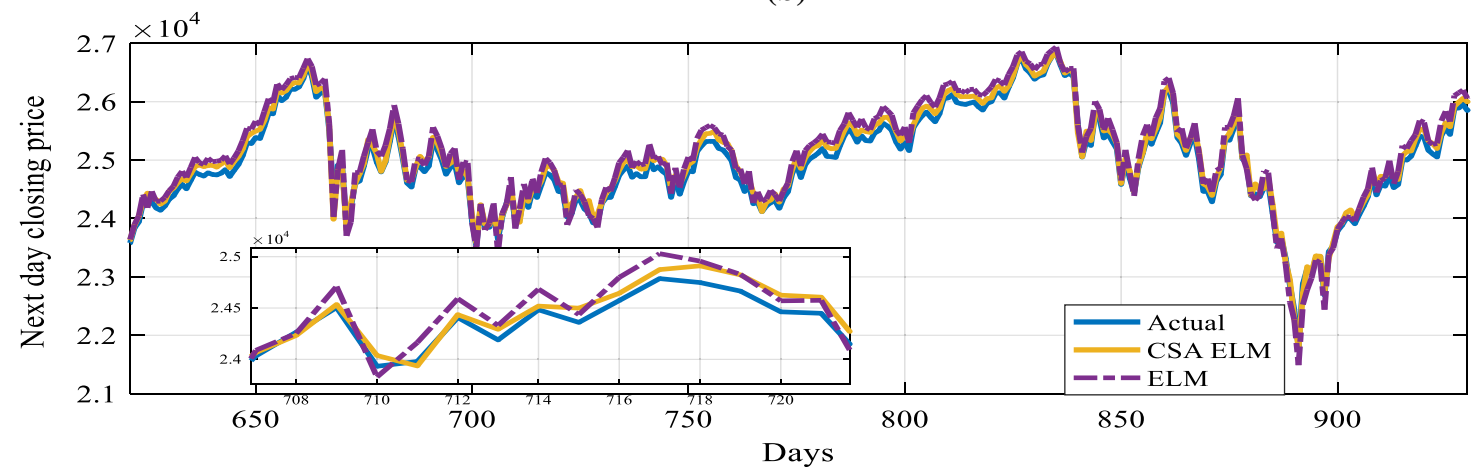

(c)

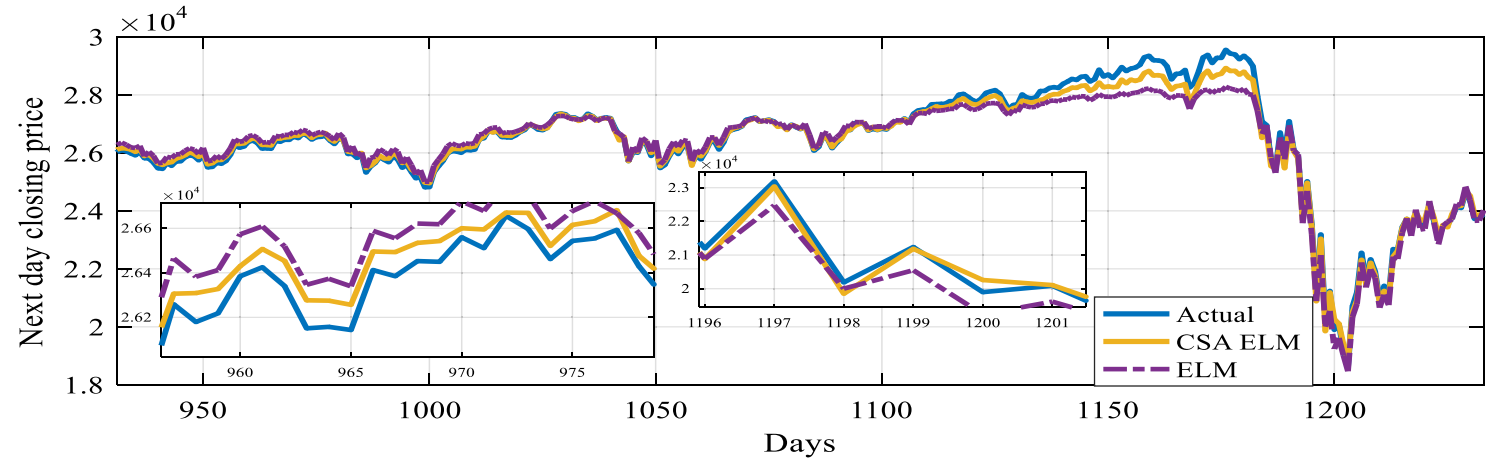

(d)

Fig. 9 CSA ELM and ELM models predicted next day closing price of DJI 

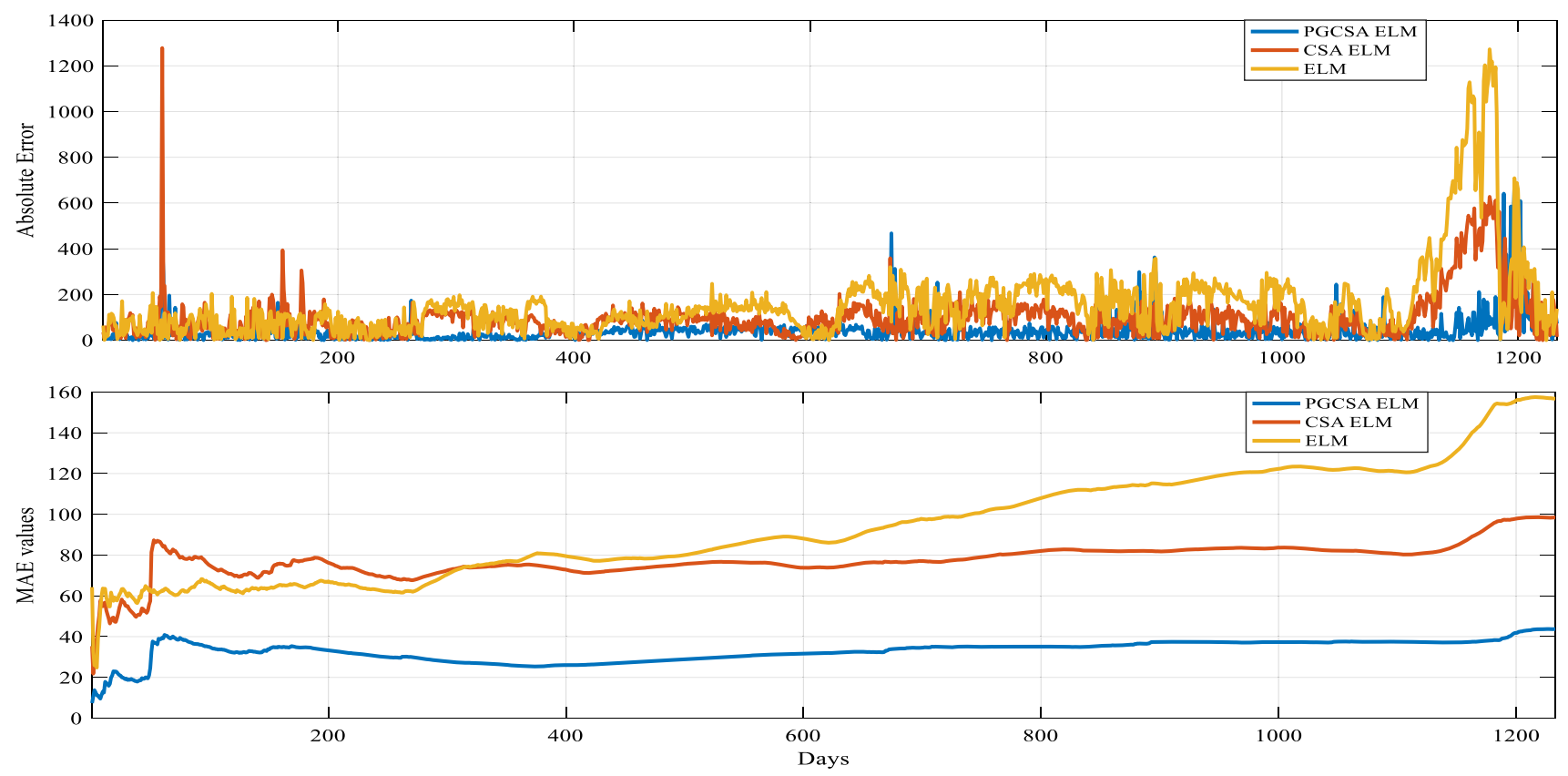

Fig. 10 absolute error and MAE values of predicted DJI closing price

\subsection{Performance measures}

Performance measure is a measurement which numerically quantifies the closeness of predicted data to actual data. Mean squared error (MSE), Mean Absolute Error (MAE) and Mean Absolute Percentage Error (MAPE) are used as error measurements which indicate error measured from the origin and accuracy in percentage respectfully. Minimum MSE, MAE and MAPE values indicate better predicted data. MSE, MAE and MAPE are the performance measures expressed in Eqs. (17)-(19), respectively.

$\mathrm{MSE}=\frac{1}{N_{\text {test }}} \sum_{i=1}^{N_{\text {test }}}\left(s_{i}-\widehat{s_{i}}\right)^{2}$

$\mathrm{MAE}=\frac{1}{N_{\text {test }}} \sum_{i=1}^{N_{\text {test }}}\left|s_{i}-\widehat{s_{i}}\right|$

MAPE $=\frac{1}{N_{\text {test }}} \sum_{i=1}^{N_{\text {test }}}\left|\frac{s_{i}-\widehat{s_{i}}}{\widehat{s_{i}}}\right| \times 100$

where, $\mathrm{N}_{\text {test }}$ is the numbers of data to be tested. $s_{i}$ and $\widehat{s_{i}}$ are the actual and predicted closing prices, respectively. In this work, MSE is used as functional value to be minimized by optimizing the weights and biases of ELM model. The performance measures used for this purpose are shown in Eqs. (20)-(23).
Mean Arctangent Absolute Percentage Error(MAAPE)

$$
=\frac{1}{N_{\text {test }}} \sum_{i=1}^{N_{\text {test }}}\left(A A P E_{i}\right)
$$

where, $A A P E_{i}=\arctan \left(\left|\frac{S_{i}-\widehat{S_{i}}}{S_{i}}\right|\right)$

CoefficientofVariation $(\mathrm{CoV})=\frac{\text { StandardDeviation }}{\text { Mean }} \times 100$

Corelations $(\mathrm{CORR})=\frac{\sum_{i=1}^{N_{\text {test }}}\left(S_{i}-\text { mean }\left(S_{i}\right)\right)\left(\widehat{S}_{i}-\operatorname{mean}\left(\widehat{S}_{i}\right)\right)}{\sqrt{\sum_{i=1}^{N_{\text {eest }}}\left(S_{i}-\operatorname{mean}\left(S_{i}\right)\right)^{2} \sum_{i=1}^{N_{\text {test }}}\left(\widehat{S}_{i}-\text { mean }\left(\widehat{S}_{i}\right)\right)^{2}}}$

Theil's U $=\frac{\sqrt{\frac{1}{N_{\text {test }}} \sum_{i=1}^{N_{\text {test }}}\left(S_{i}-\widehat{S}_{i}\right)^{2}}}{\sqrt{\frac{1}{N_{\text {test }}} \sum_{i=1}^{N_{\text {test }}}\left(S_{i}\right)^{2}}+\sqrt{\frac{1}{N_{\text {test }}} \sum_{i=1}^{N_{\text {test }}}\left(\widehat{S}_{i}\right)^{2}}}$

$\mathrm{CoV}$ is useful performance measures to interpret a difference between two predicted data sets. The $\mathrm{CoV}$ demonstrates the variability of data in a sample in relation to the mean of the population. In this work, $\mathrm{CoV}$ is used to measure the volatility and risk in compare to return. MAAPE is a scale-independent and interpretability performance measure to estimate forecast accuracy. It achieves extra balanced penalty of errors over MAPE [56]. CORR is a statistical measure which determines the relative movements of actual and predicted closing price. The 


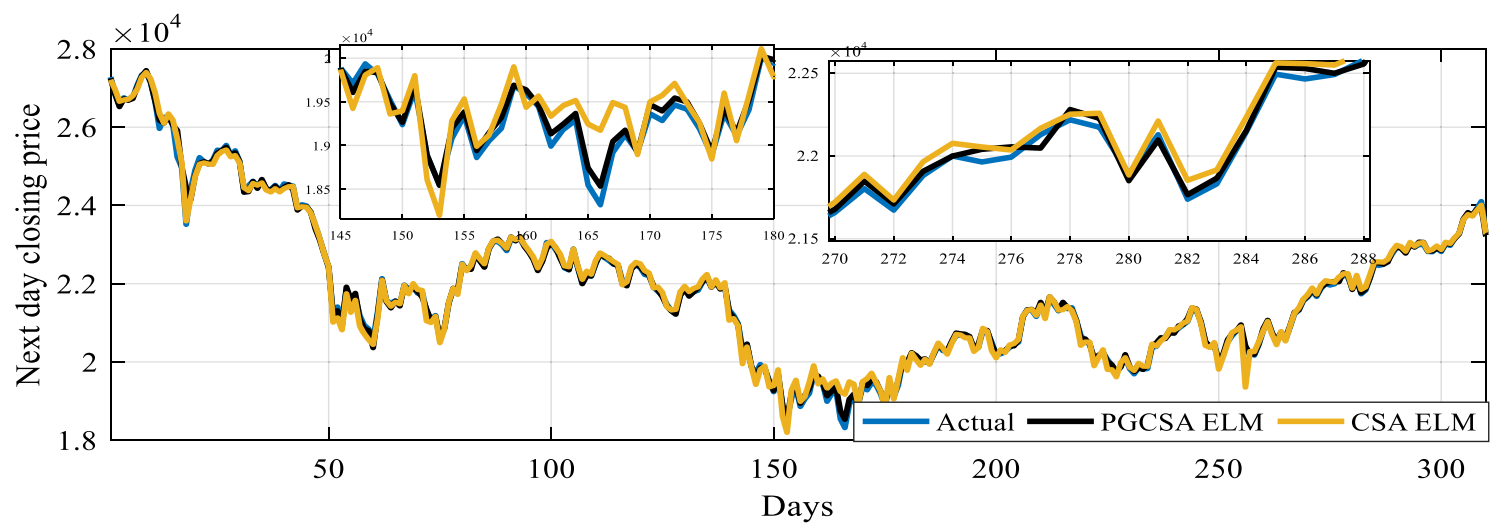

(a)

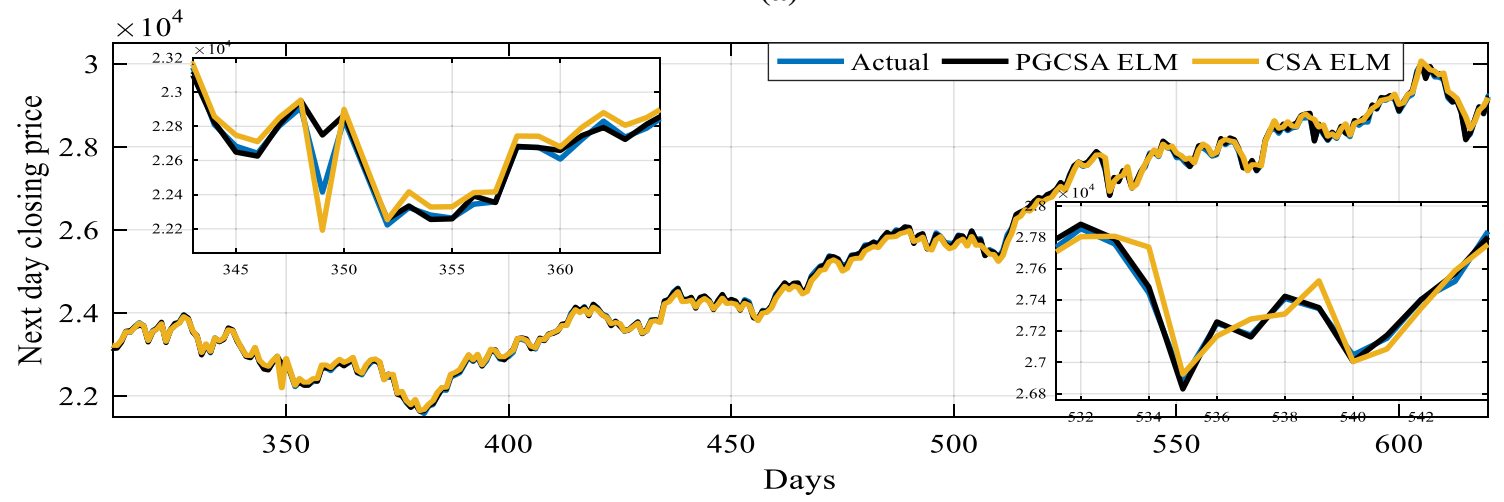

(b)

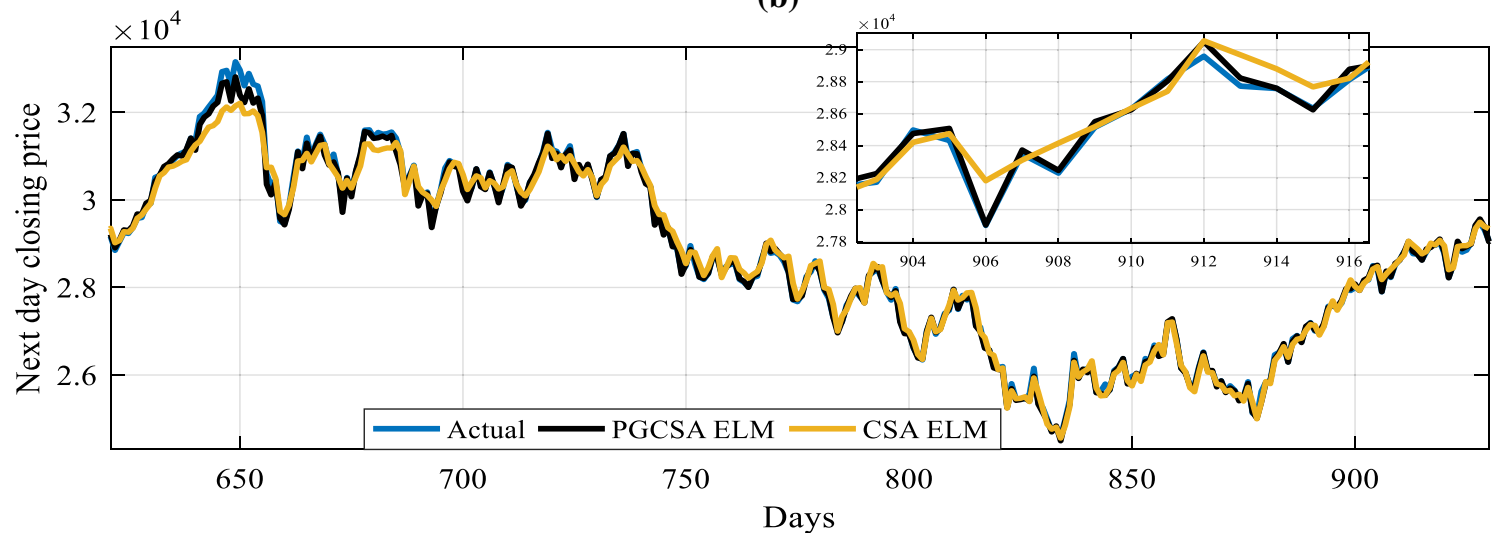

(c)

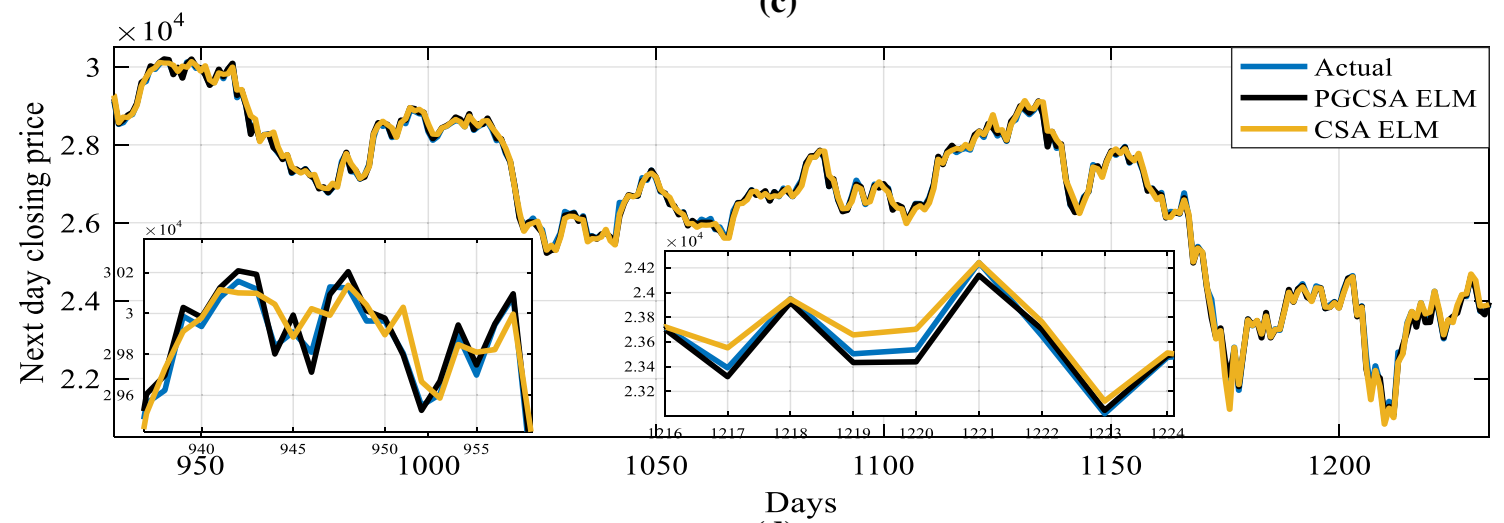

(d)

Fig. 11 PGCSA ELM and CSA ELM models predicted next day closing price of HSI 


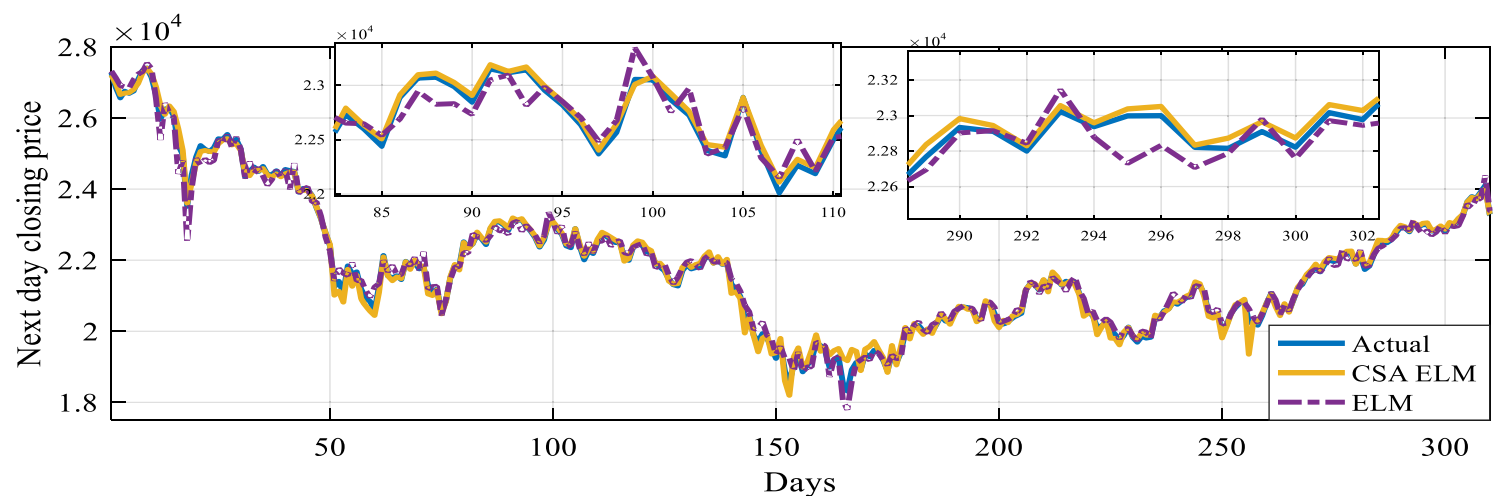

(a)

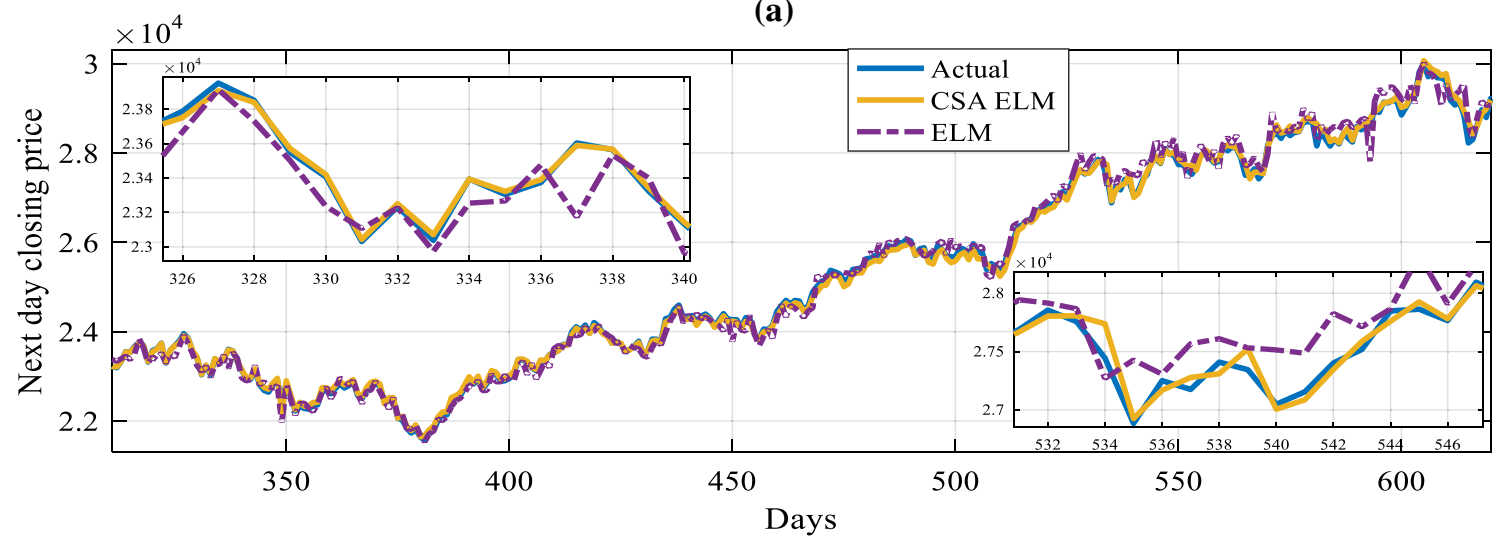

(b)

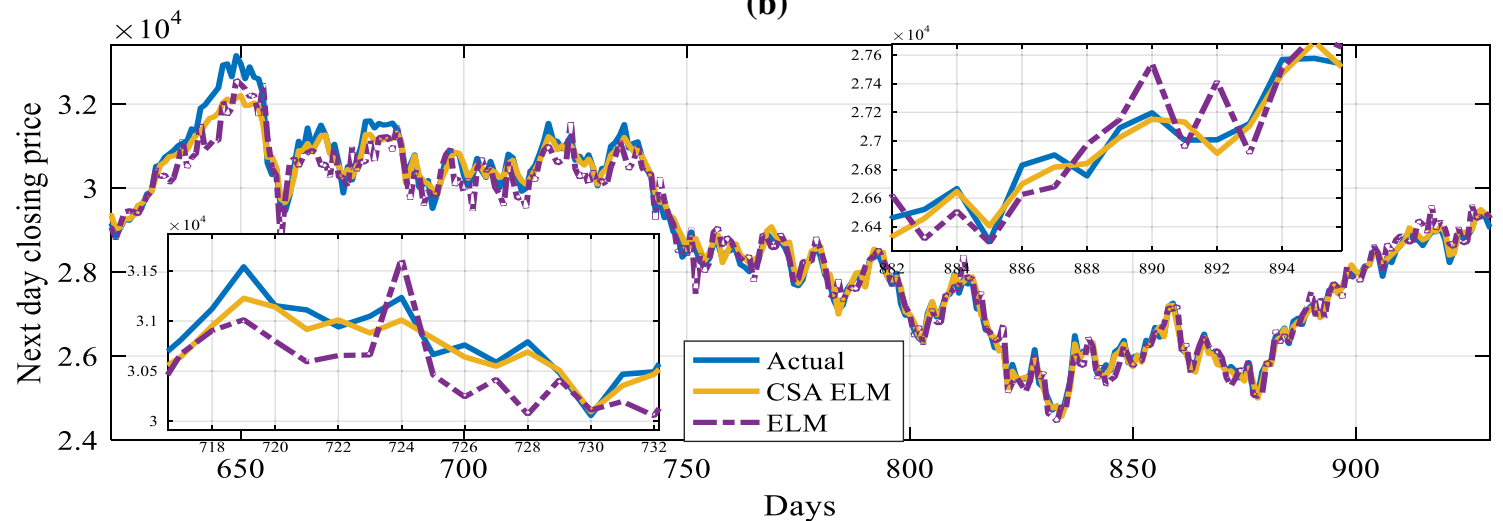

(c)

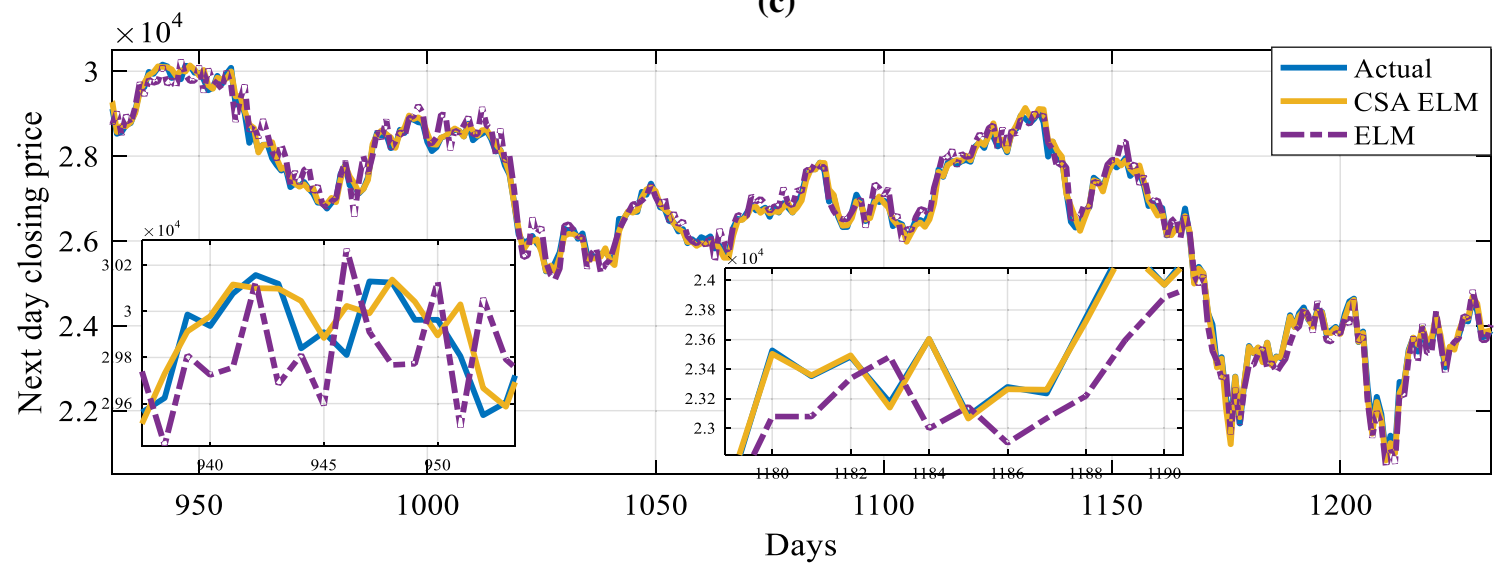

(d)

Fig. 12 CSA ELM and ELM models predicted next day closing price of HSI 


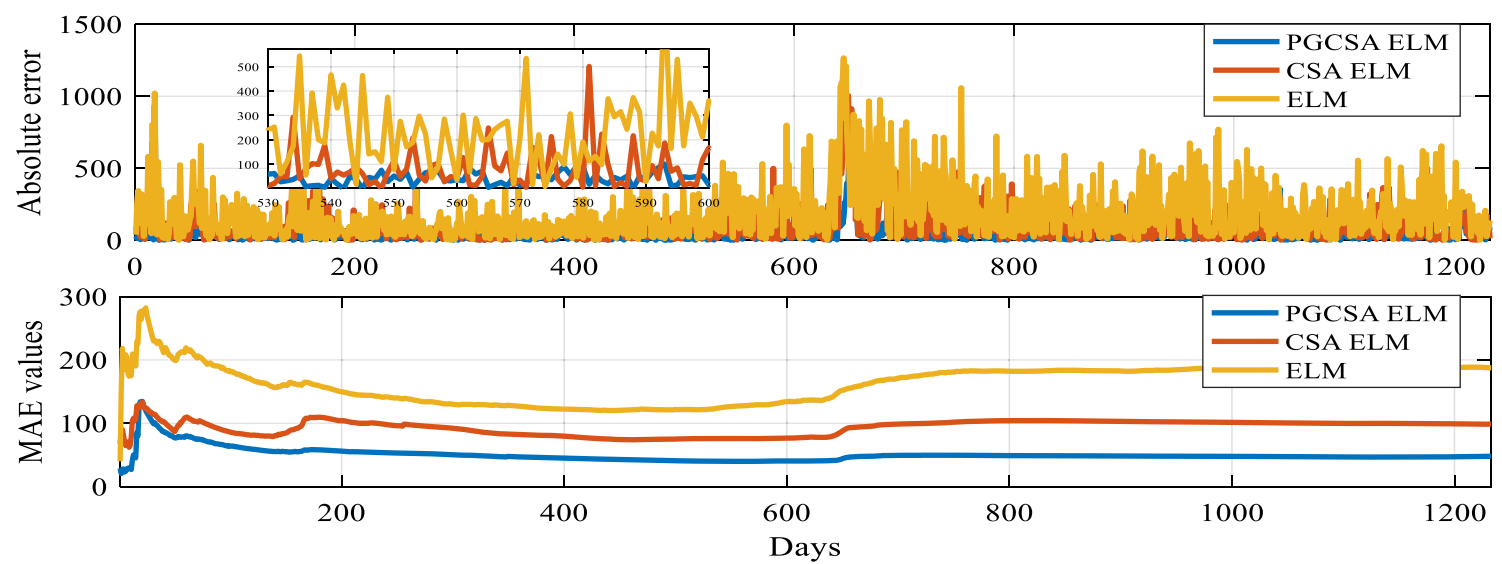

Fig. 13 absolute error and MAE values of predicted HSI closing price

range of correlation coefficient is between -1 to 1 . The positive correlation coefficient near to 1 (CORR $\ngtr 1)$ provides a strong linear relationship between actual and predicted closing price. Theil's $U$ is used as a statistical measure to determine the forecasting accuracy. The smaller value of Theil's U indicates more accuracy of forecast.

\subsection{Hypothesis testing (paired t-test)}

In this work, paired t-test is used to hypothetically test the accuracy of predicted closing price. Paired t-test requires two samples of observations such as predicted price $\left(\widehat{S_{i}}\right)$ and actual price $\left(S_{i}\right)$ with n samples. The t-test performs to state the acceptance of null $\left(\mathrm{H}_{0}\right)$ and alternative $\left(\mathrm{H}_{1}\right)$ hypotheses. The null and alternative hypotheses adopted for this work are:

$\mathrm{H}_{0}$ - The mean difference is equal to zero $\left(\mu_{S_{i}}=\mu \widehat{\widehat{S}_{i}}\right)$.

$\mathrm{H}_{1}$ - The mean difference is not equal to zero $\left(\mu_{S_{i}} \neq \mu_{\widehat{S_{i}}}\right)$.

The paired t-test is mathematically described in Eqs. (24)-(27).

dif $=S_{i}-\widehat{S}_{i}$

$\overline{\text { dif }}=\frac{\sum d i f}{n}$

$S D=\sqrt{\frac{\sum(\text { dif }-\overline{\text { dif }})^{2}}{n-1}}$

$t-$ value $=\sqrt{n} \frac{\overline{d i f}}{S D}$

where, dif, $\overline{d i f}$, and SD are the difference, mean of difference, and sample standard deviation, respectively. In this work, the acceptance/failure of acceptance of null hypothesis is decided by conceding the significance level of $5 \%(0.05)$.

\subsection{Sharpe ratio (SR) and modified sharpe ratio (MSR)}

An investor requires nimble investment to achieve good return with less risk. For the purpose to help investors to understand the return of an investment compared to its risk, sharpe ratio is an useful financial tool. The mathematical expression of SR is defined in Eq. (28).

$S R=\frac{R_{P}-R_{F}}{\sigma_{p}}$

where, $\mathrm{R}_{\mathrm{P}}, \mathrm{R}_{\mathrm{F}}$ and $\sigma_{p}$ are the portfolio return, risk free rate and standard deviation of portfolio return, respectively. Modified Sharpe ratio (MSR) is a modified tool of SR, which encourages that any abnormalities (abnormal distributed assets) are precluded from its calculation. Modified Sharpe Ratio (MSR) is used for a statistical analysis by concerning Modified Value at Risk (MVaR). MVaR measures the level of risk within a portfolio with non-normal distribution return of a specific time [57]. MSR and MVaR are determined as illustrated in Eq. (29) and (30).

$$
\begin{aligned}
M S R= & \frac{R_{P}-R_{F}}{M V a R} \\
M V a R= & W\left[\mu-\left\{Z_{c}+\frac{1}{6}\left(Z_{c}^{2}-1\right) S+\frac{1}{24}\left(Z_{c}^{3}-3 Z_{c}\right) K\right.\right. \\
& \left.\left.-\frac{1}{36}\left(2 Z_{c}^{3}-5 Z_{c}\right) S^{2}\right\} \sigma\right]
\end{aligned}
$$




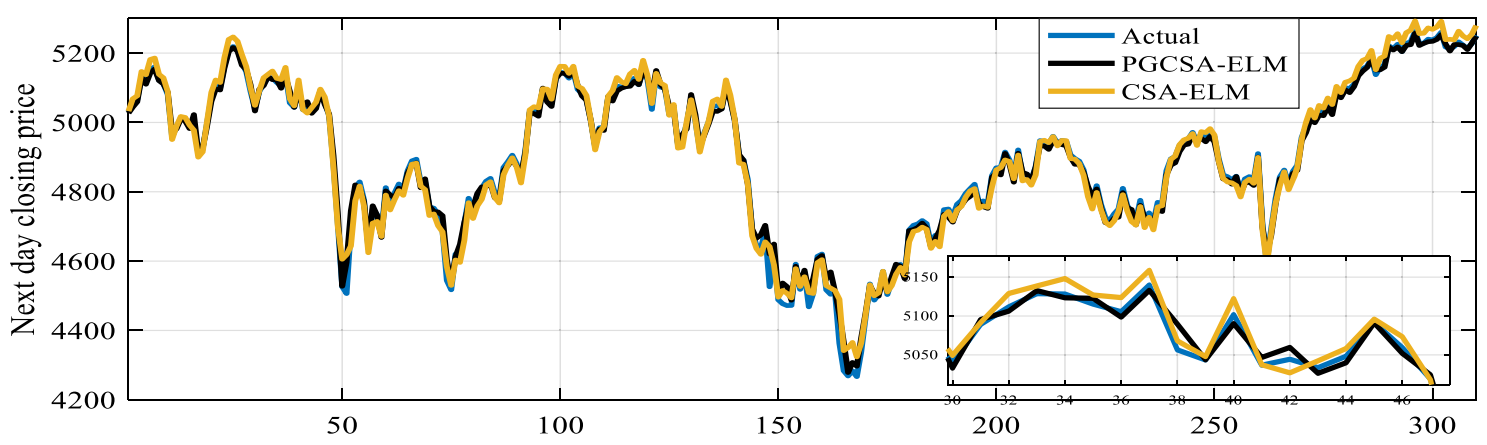

(a)

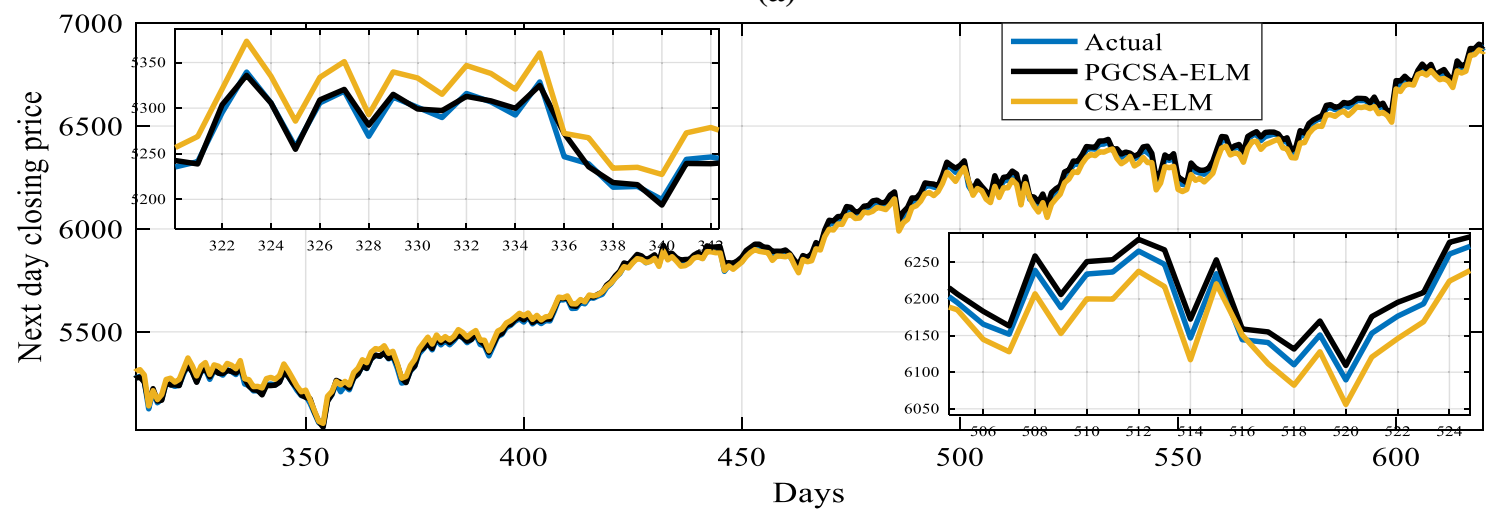

(b)

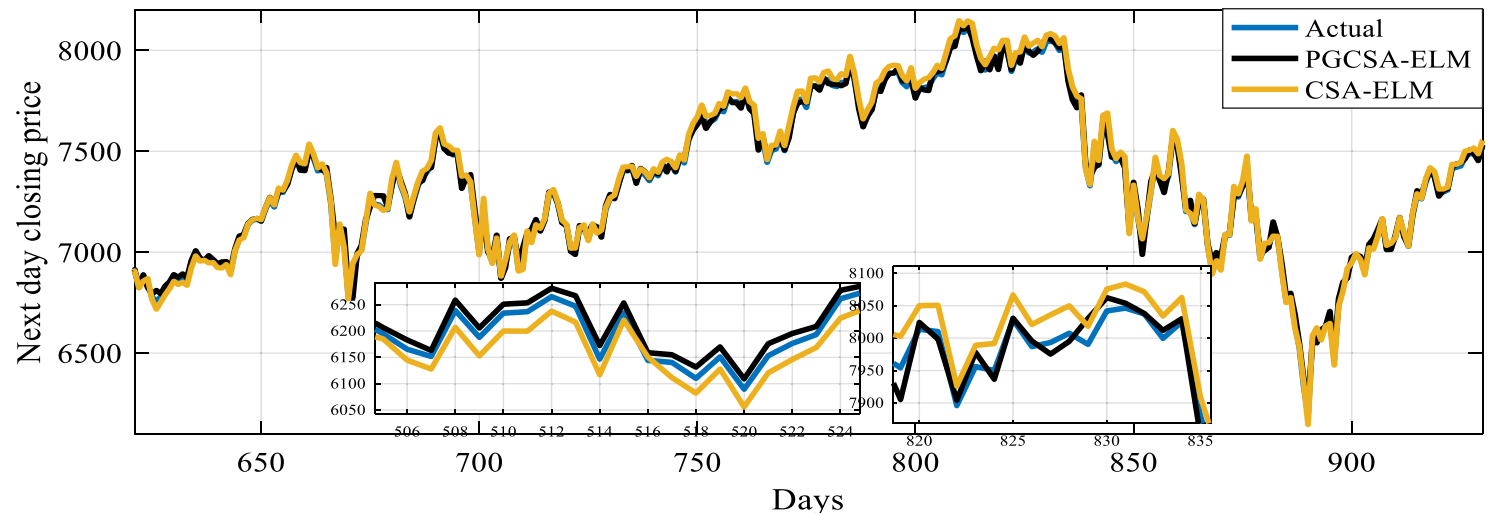

(c)

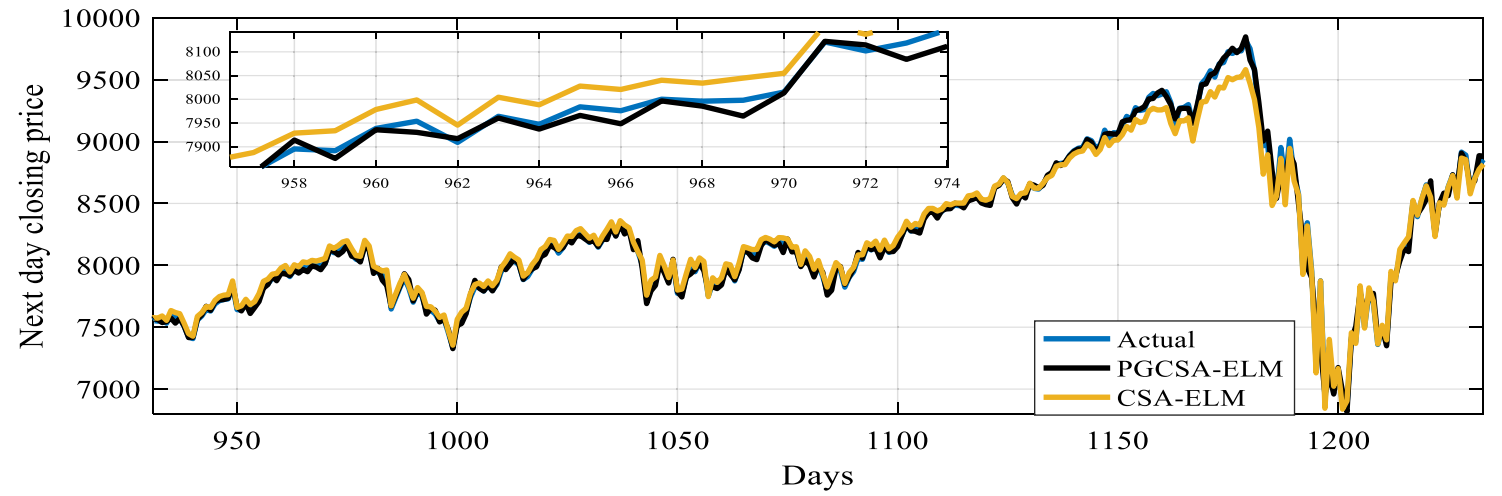

(d)

Fig. 14 PGCSA ELM and CSA ELM models predicted next day closing price of IXIC 


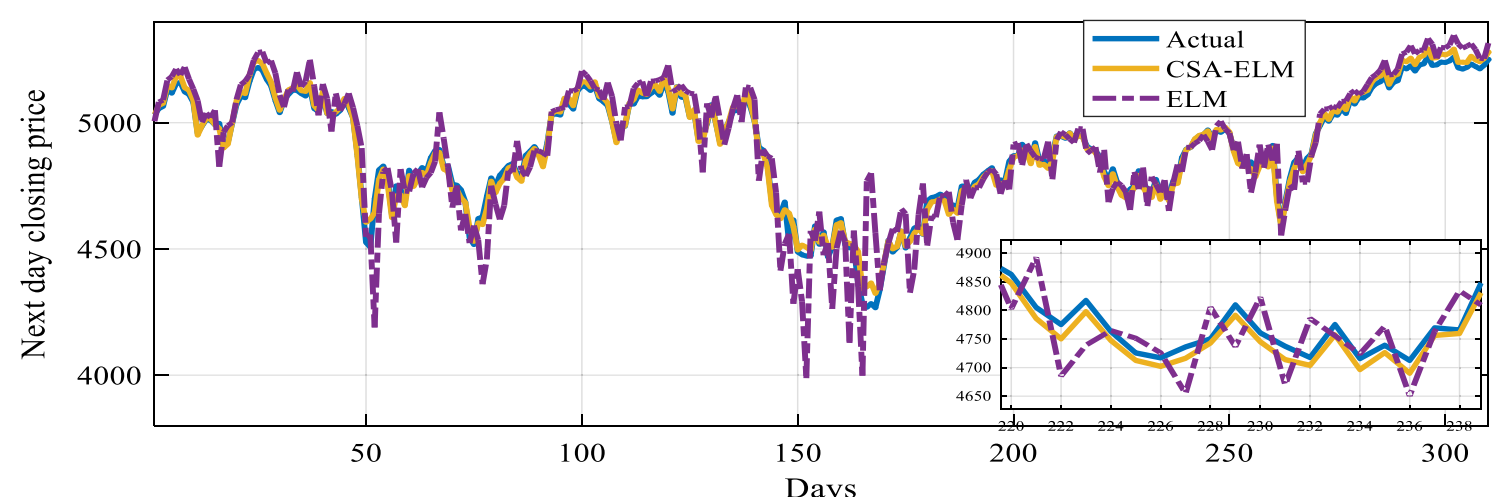

(a)

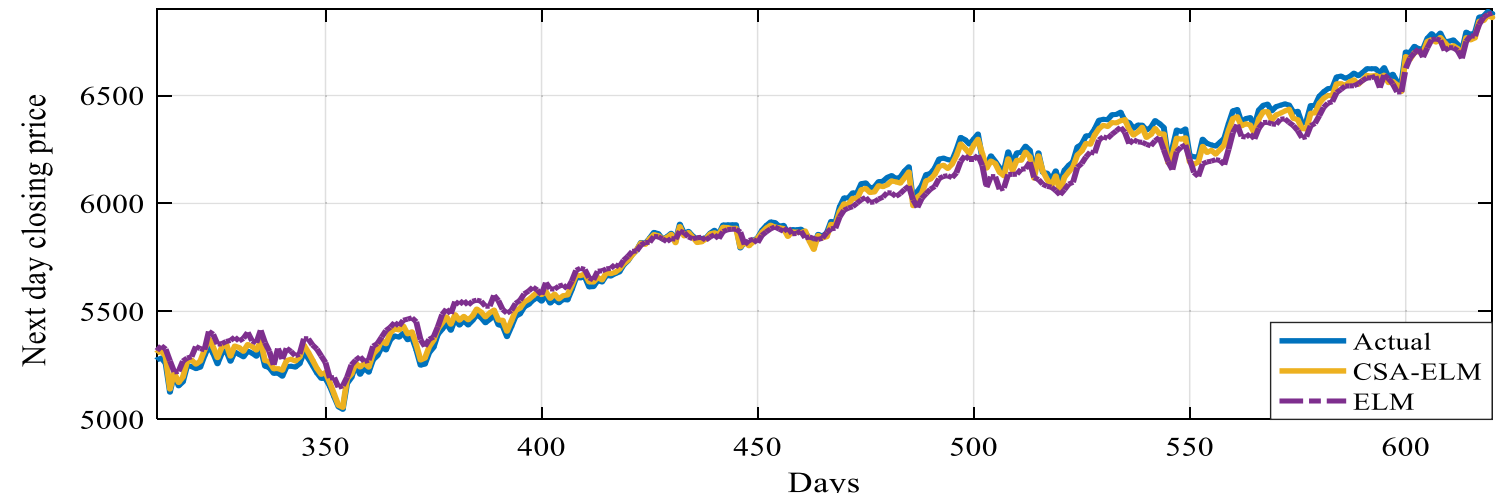

(b)

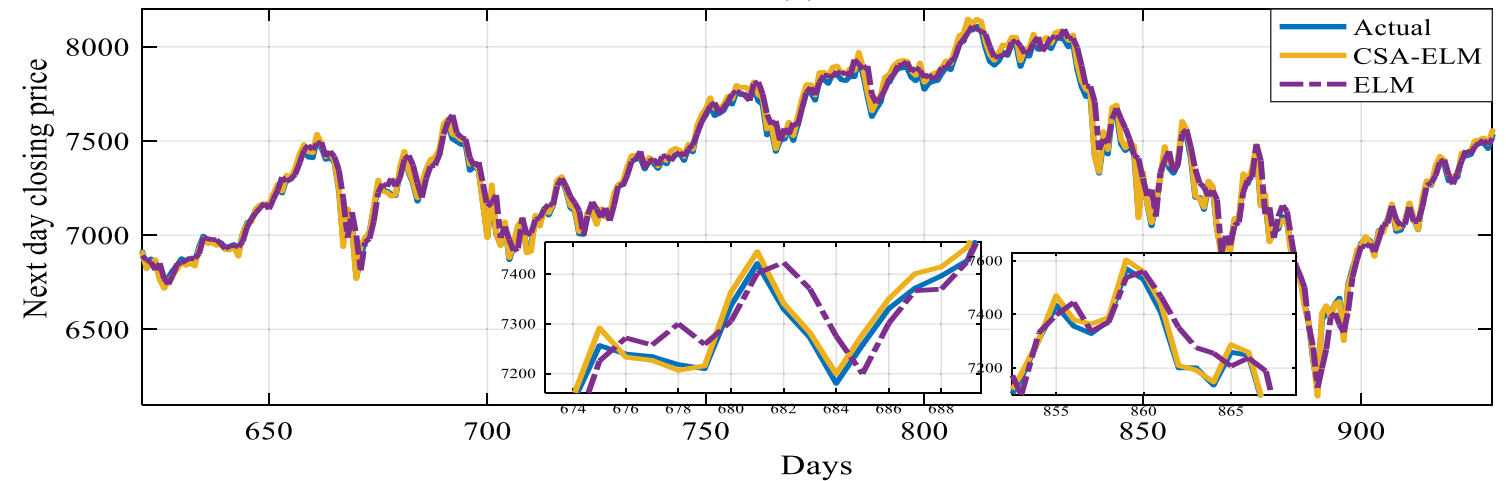

(c)

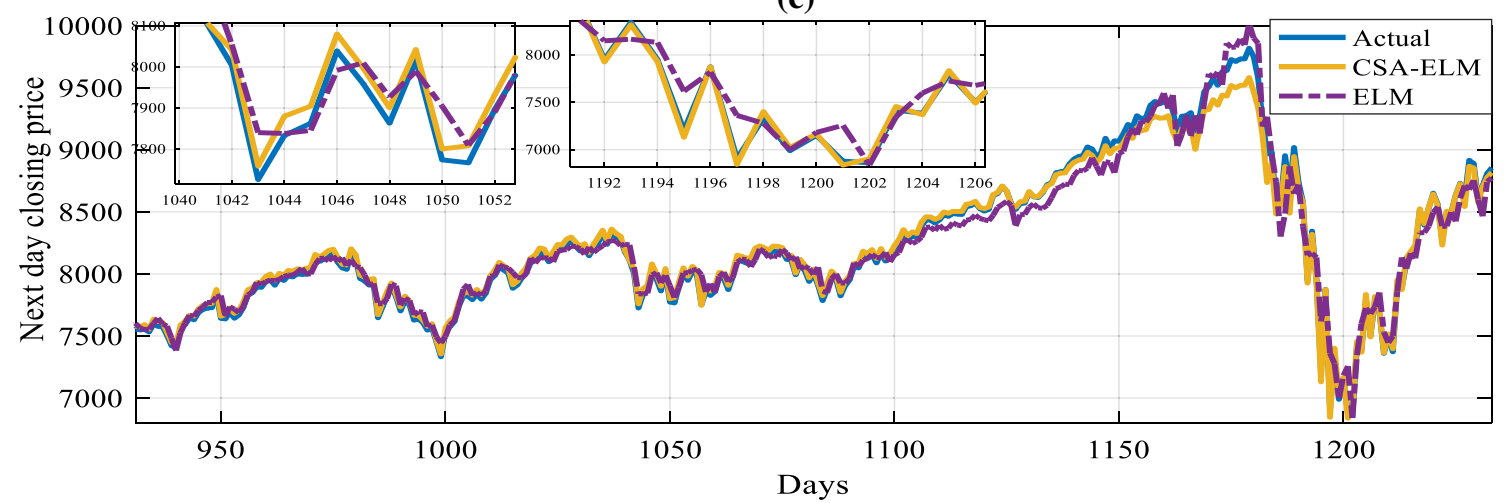

(d)

Fig. 15 CSA ELM and ELM models predicted next day closing price of IXIC 


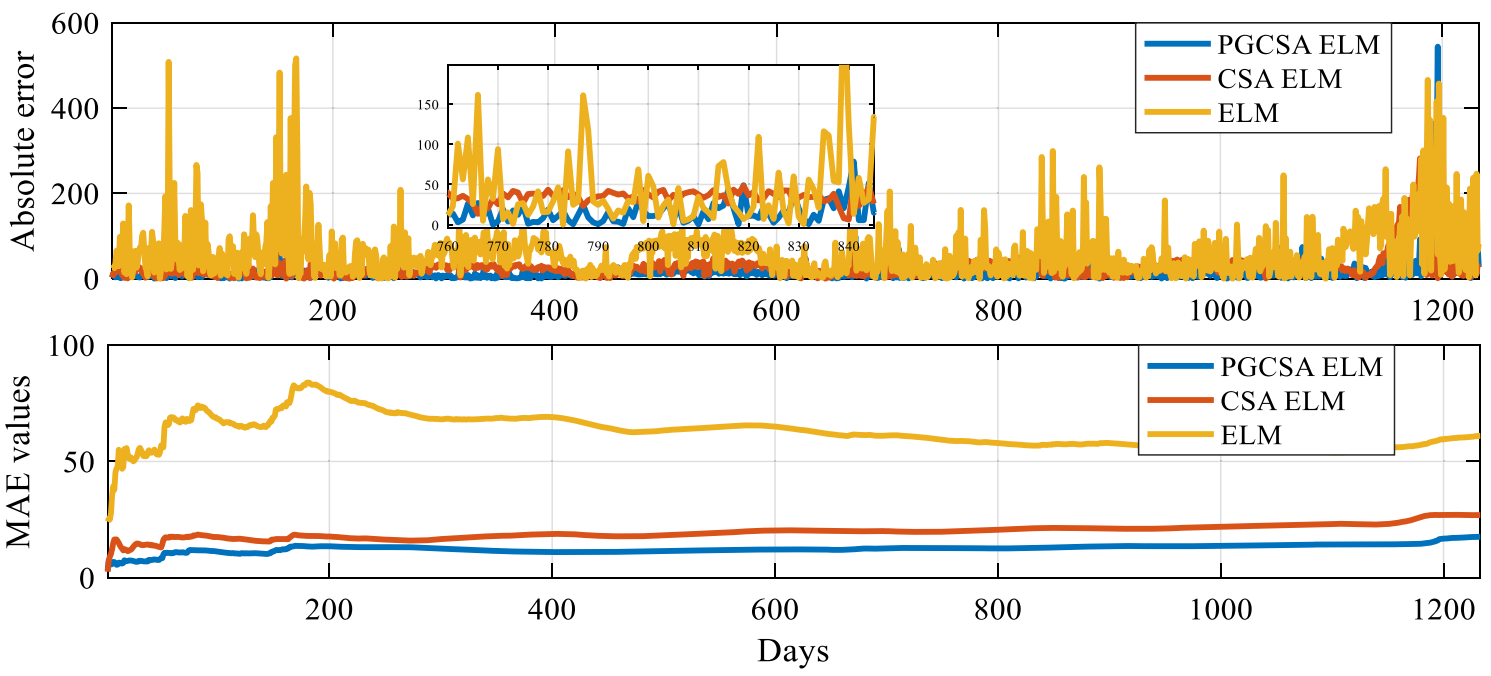

Fig. 16 Absolute error and MAE values of predicted IXIC closing price

where, $Z_{c}=$ Critical value for probability. $S=$ Skewness. $\mathrm{K}=$ Excess kurtosis. $\mu=$ Rate of drift of asset value. $\mathrm{W}=$ Amount at risk or portfolio.

\section{Result and discussion}

\subsection{Validation of proposed algorithm through benchmark test functions}

The novelty of CSA is favorably demonstrated in various engineering applications. This algorithm has certain kind of scarcity to solve complex problems. CSA largely depends on random position of crows and randomly selected crow. The position of a crow may be updated by conceding unfit and worst crow which is not acceptable. The sluggish convergence rate and probability to trap into local optima may be caused by this dilemma. The competence of CSA algorithm is enhanced by modifying the mathematical expression of the algorithm. The proposed PGCSA algorithm is demonstrated in this work in contrast with DE [42], PSO [44], Teaching Learning-Based Optimization (TLBO) [58], Salp Swarm Algorithm (SSA) [59] and CSA [48] algorithms. The competence of PGCSA algorithm to pluck optimum point, convergence rate and evading from local optima is portrayed by an admirable comparative analysis among recently proposed algorithms. The comparative analysis is observed by solving 9 benchmark functions without constraint and 3 benchmark functions with constraints. All the algorithms are executed individually for individual benchmark equations with same population and termination criteria
For all algorithms, population size and maximum iterations are chosen as 20 and 1000, respectively. The solutions of each benchmark equations are evaluated by executing each algorithm for 50 times. The best solution among 50 runs is considered as the solution of the corresponding algorithms. The benchmark functions adopted for this work are categorized as unimodal separable/non-separable (US/UN) and multimodal separable/non separable (MS/MN). The constraint and unconstraint benchmark equations are tabulated in Tables 1 and 2 respectively. The different categories of functions are adopted to contribute a fine validation of proposed algorithm in different environments. The Best value (BV), Average value (Avg) and Standard Deviation (SD) of the solution are opted as performance parameters. The comparative analysis is graded by conceding these parameters. All the adopted benchmark functions are minimization problems. The solutions of benchmark problems without constraints are portrayed in Table 3 and the solutions of benchmark problems with constraints are portrayed in Table 4 . The convergence plots of each algorithm of benchmark problems without constraints are illustrated in Fig. 4 and the convergence of benchmark problems with constraints are portrayed in Fig. 5. From Figs. 4, 5 and Tables 3, 4, the proficiency of proposed PGCSA algorithm along with faster convergence and capability to escape from local optima are validated over CSA, SSA, TLBO, PSO and DE algorithms. From Tables 3 and 4, the performance parameters contributed by PGCSA of almost all benchmark functions are favorably less. The convergence rate of PGCSA algorithm is also validated from Figs. 4 and 5 for first 100 iterations.

The better standard deviation and mean values of an algorithm demonstrate that the average performance and 


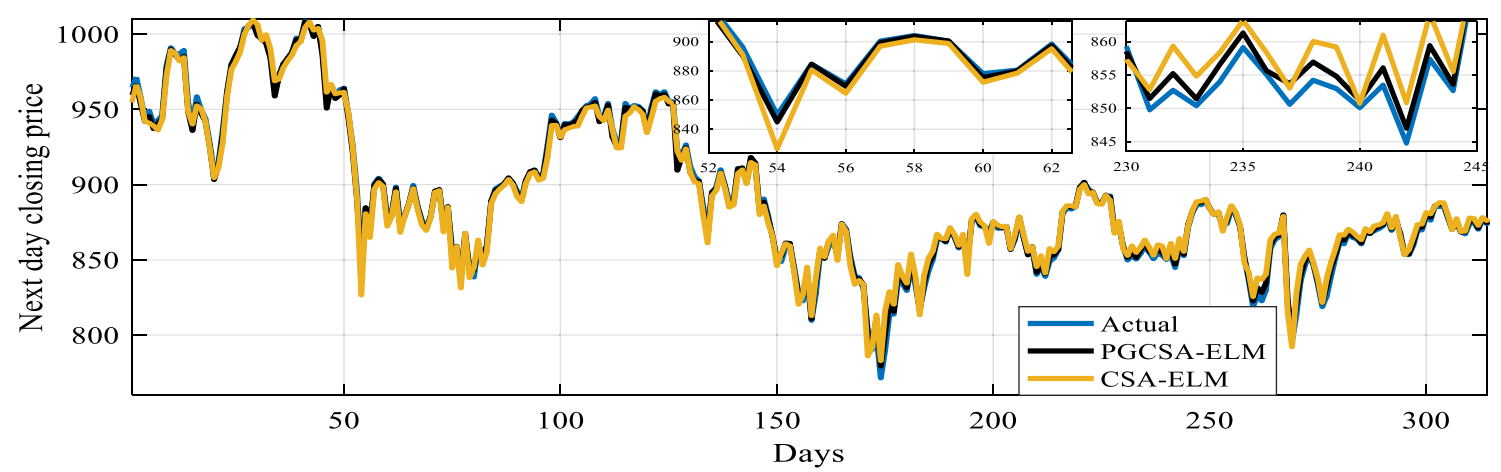

(a)

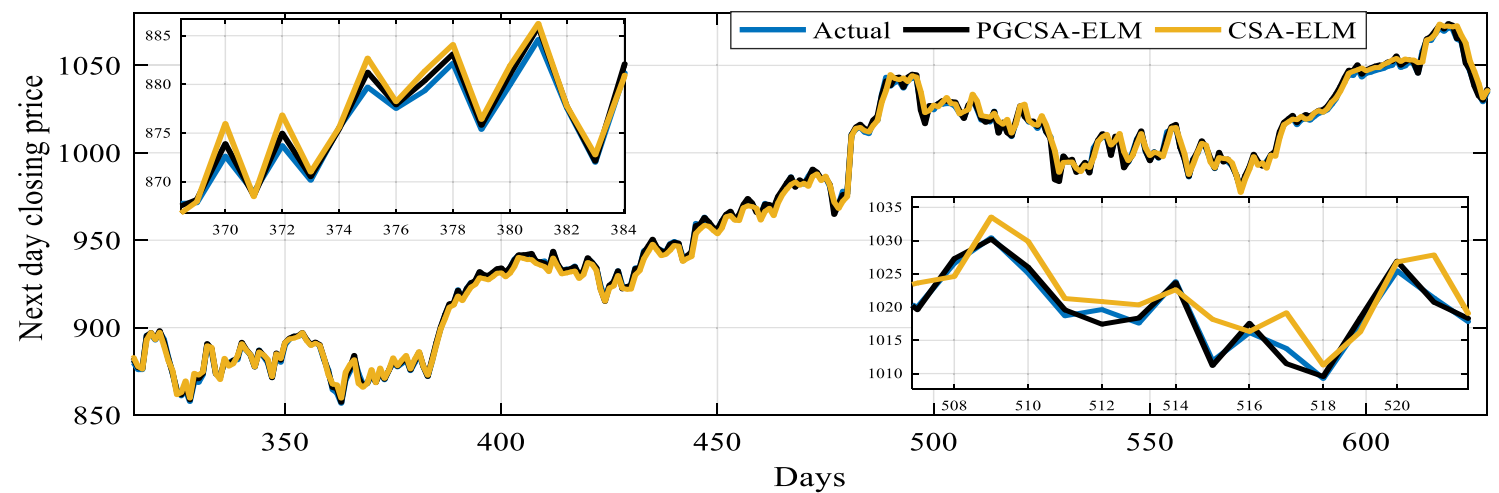

(b)

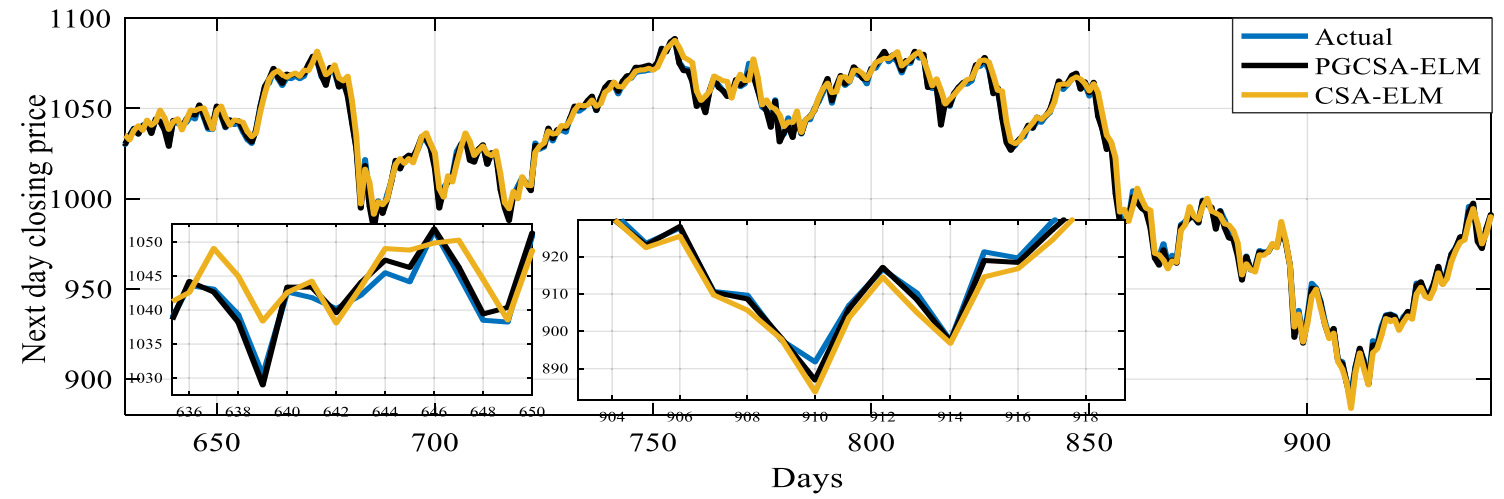

(c)

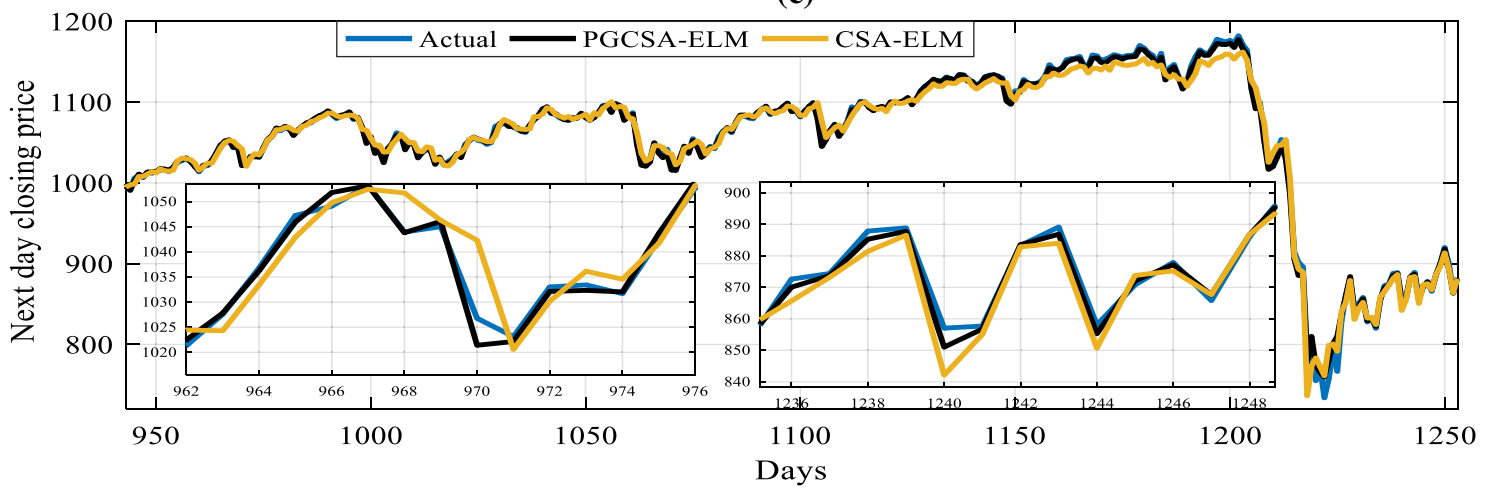

(d)

Fig. 17 PGCSA ELM and CSA ELM models predicted next day closing price of N100 


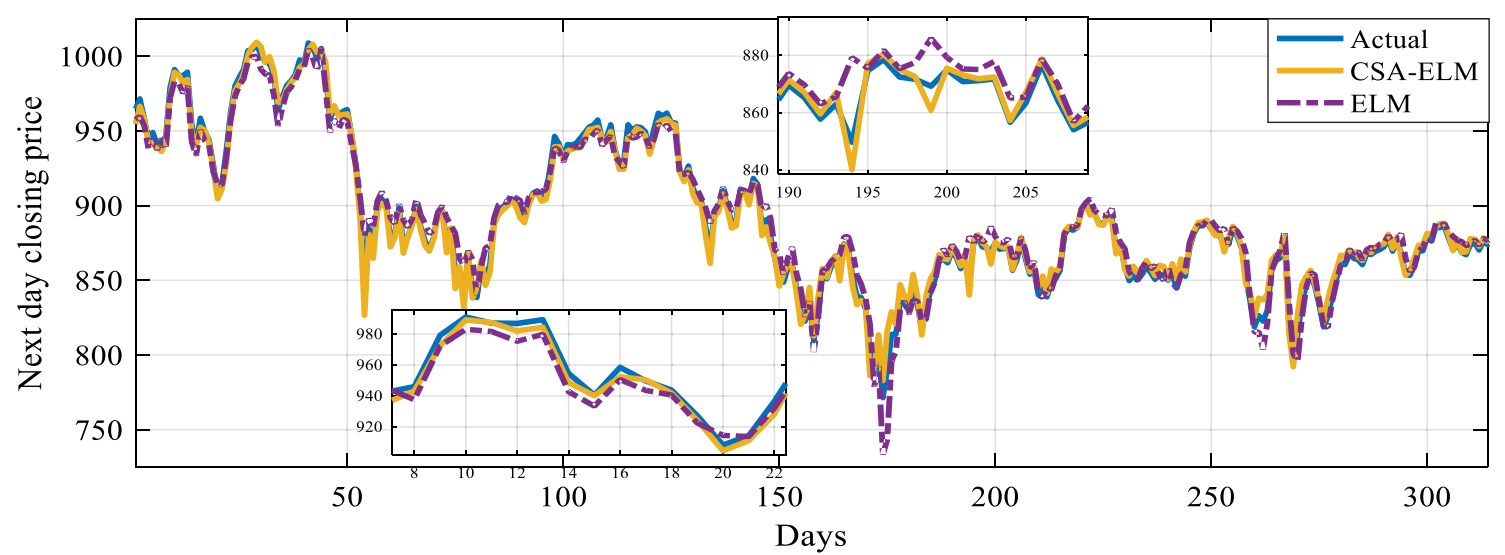

(a)

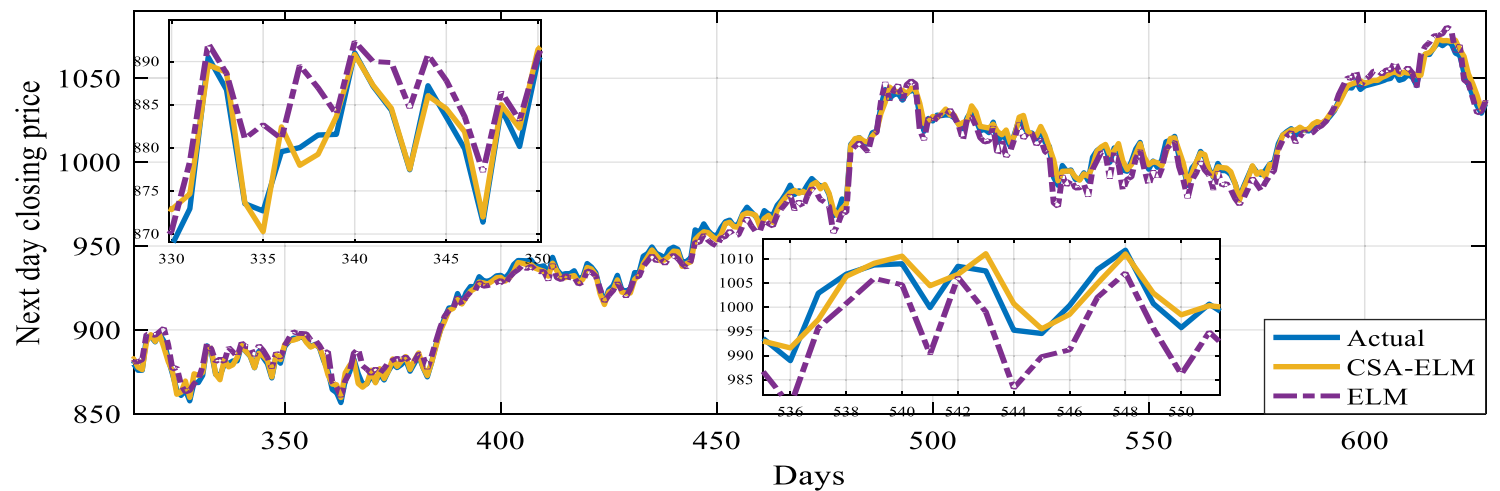

(b)

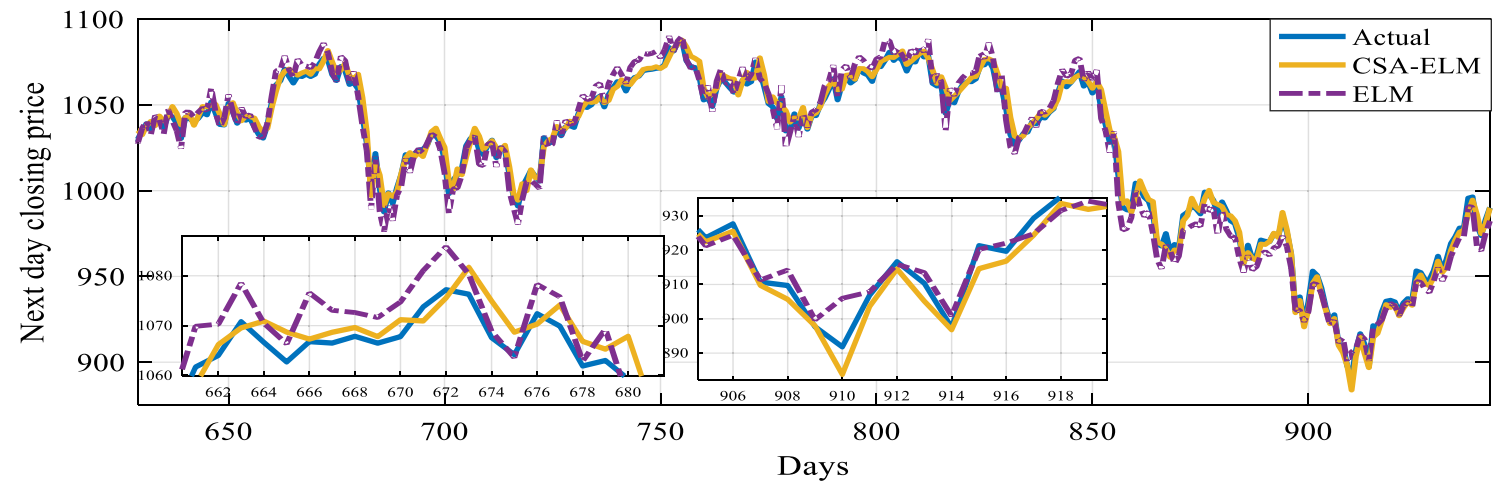

(c)

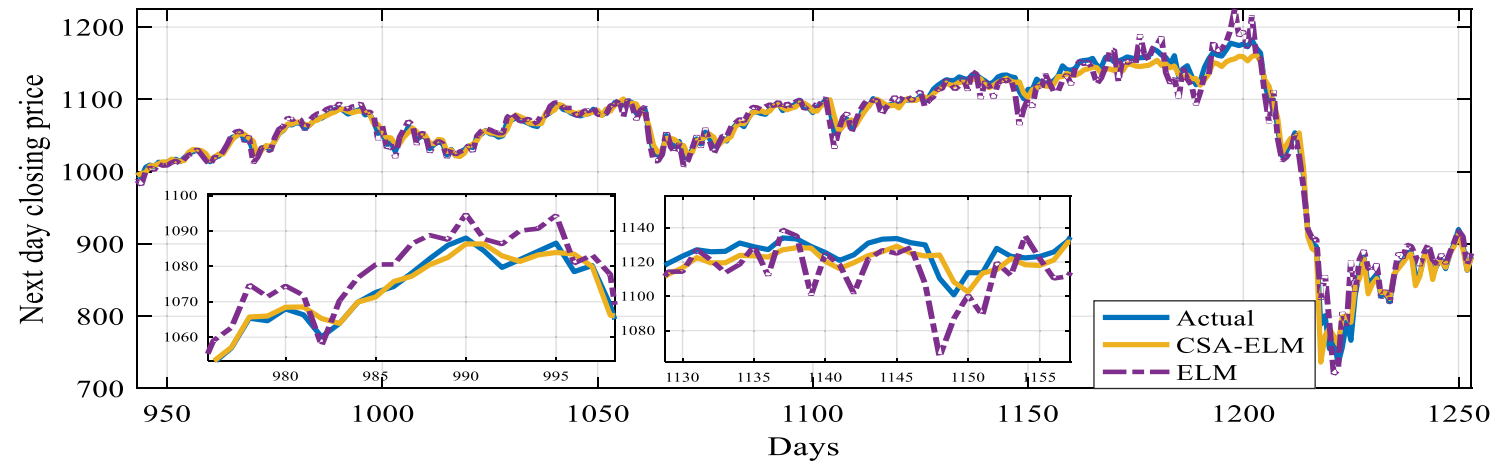

(d)

Fig. 18 CSA ELM and ELM models predicted next day closing price of N100 

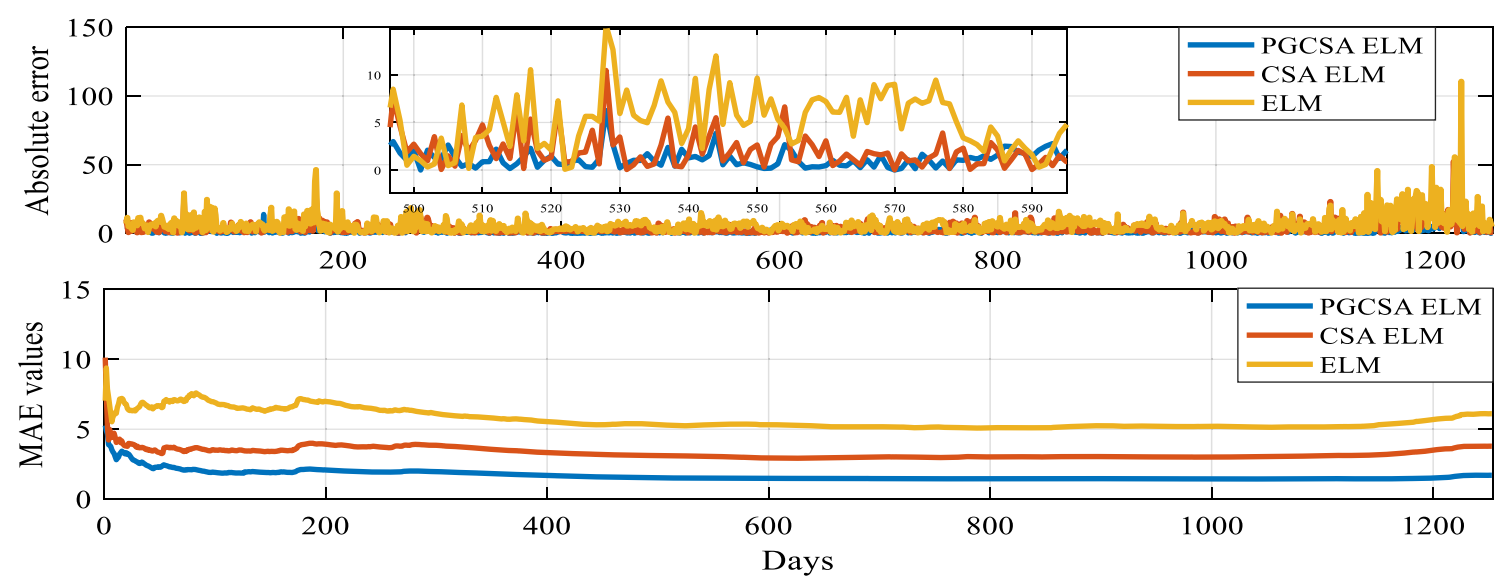

Fig. 19 Absolute error and MAE values of predicted N100 closing price

stability of proposed PGCSA algorithm is better over other considered algorithms. In addition to mean and standard deviation, a pairwise hypothesis test is conducted by using Wilcoxon signed-rank test. Null hypothesis $\left(\mathrm{H}_{0}\right)$ and alternative hypothesis $\left(\mathrm{H}_{1}\right)$ are considered to interpret the superiority of PGCSA algorithm.

$\left\{\begin{array}{l}H_{0}: \text { Mean difference is zero } \\ H_{1}: \text { Mean difference is not zero }\end{array}\right.$

The main concerns to reject or accept the null hypothesis are p-value and significance level $(\alpha=0.05)$. A $p$ value larger than significance level fails to reject null hypothesis, while a p-value smaller than significance level reject the null hypothesis. The $p$-values have been determined and portrayed in Table 5. From Table 5, all $p$-values are less than 0.05 which indicates the evidence to reject null hypothesis with a significance level of $95 \%$.The rank sum values have been calculated between PGCSA and other algorithms which are shown in Table $6 . \mathrm{R}+$ is the sum of positive ranks which indicates the PGCSA algorithm outperformed the other algorithm and $\mathrm{R}$ - is the sum of negative ranks which indicates the failure of PGCSA algorithm to outperform the other algorithm. From Table 6, the sum of positive ranks for all benchmark functions is higher than the sum of negative ranks which substantiates that the PGCSA outperforms other algorithms in each comparison.

\subsection{Simulation experiments for validation of proposed technique of stock market forecasting}

In previous section, the evidence to validate the efficacy of proposed PGCSA is achieved by outperforming other existing techniques. In this section, the proposed PGCSAELM technique is executed in stock market index price forecasting for analysing the effectiveness of proposed technique with some existing techniques. IXIC stock index is considered for the comparative analysis with existing techniques such as multilayer perceptron (MLP), hybrid GARCH-MLP, dynamic architecture for artificial neural networks (DAN2), GARCH-DAN2 [10]. GDAXI index is considered to achieve a performance comparison of proposed technique with existing techniques such as GA-NN, GRNN, RBE and BNNMAS proposed by Hafezi et al. [54]. The two indices are considered with exactly same data as considered in [10, 54]. The performance measures (MAE, MSE, and MAPE) of different techniques for IXIC stock index forecasting testing period are tabulated in Table 7. The PGCSA-ELM, CSA-ELM and ELM predicted IXIC index testing data are portrayed in Fig. $6 \mathrm{a}$ and MAE values of prediction is portrayed in Fig. 6b. The performance indices and predicted quarterly GDAXI index are portrayed in Table 8 and Fig. 7a. The MAE values of predicted closing price by different models are portrayed in Fig. $7 \mathrm{~b}$. From this analysis, the effectiveness of PGCSA ELM techniques is substantiated with minimum performance measures. The testing results of GDAXI index predicted by different models are portrayed in appendix. The superiority of proposed PGCSA ELM model over CSA ELM and ELM models predicted can be concluded from this analysis. 


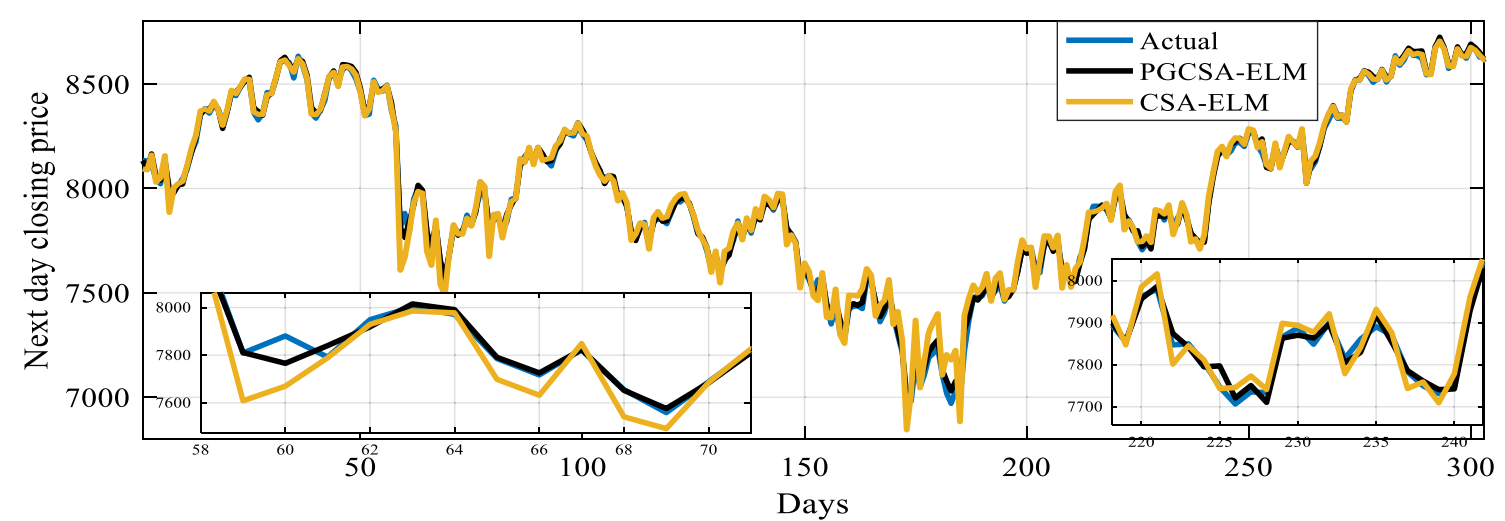

(a)

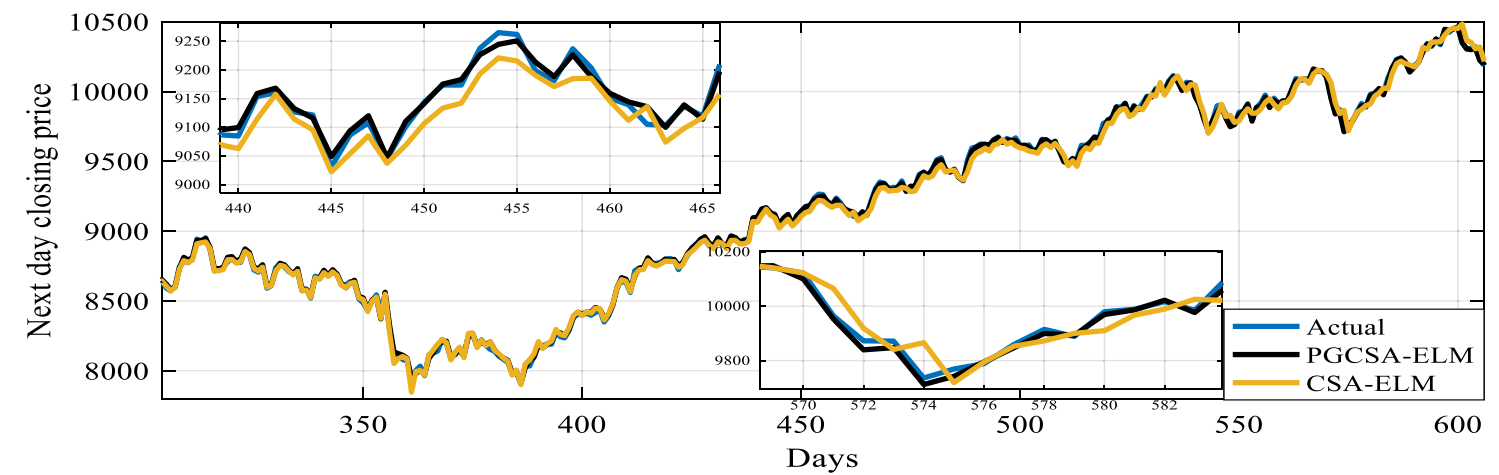

(b)

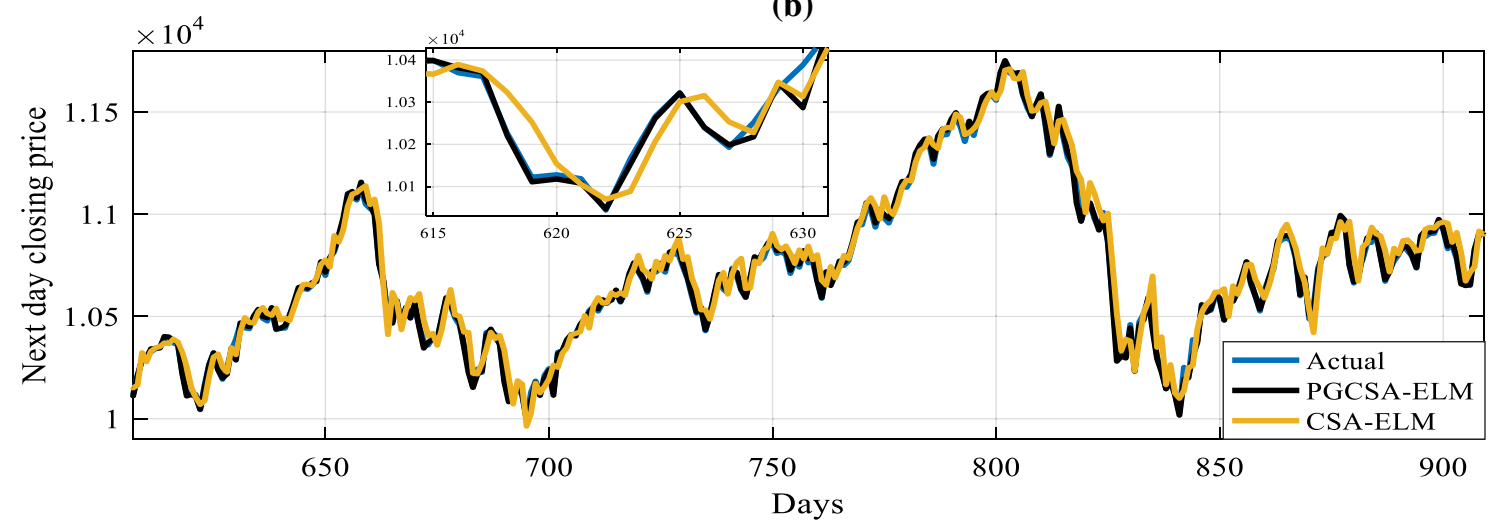

(c)

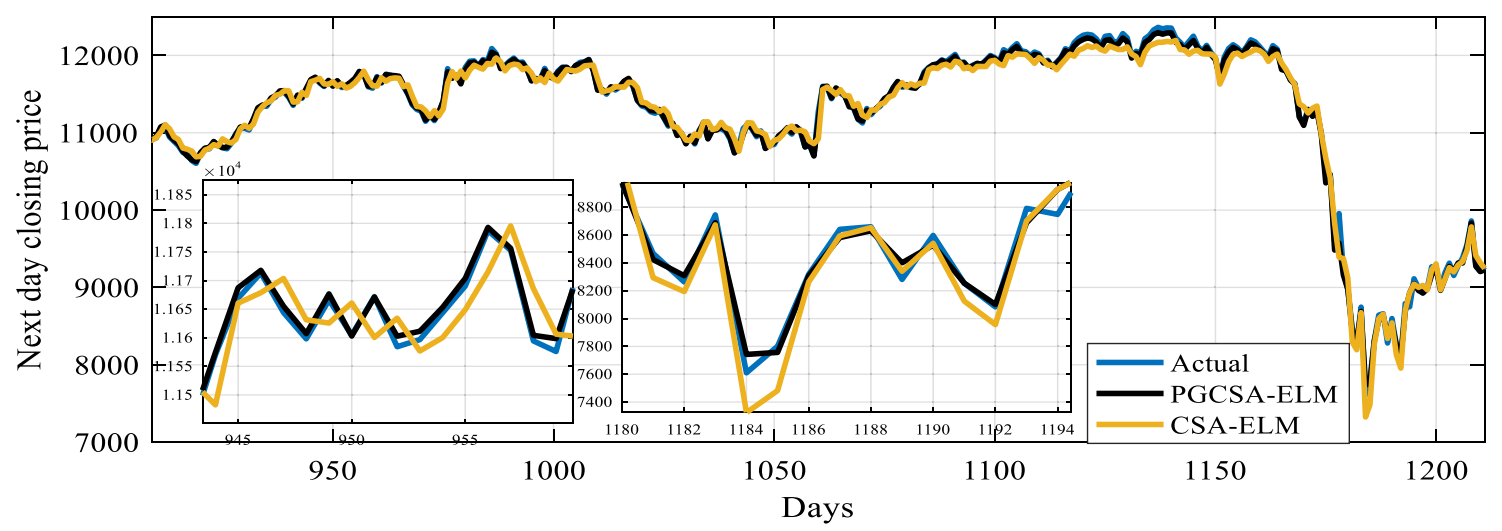

(d)

Fig. 20 PGCSA ELM and CSA ELM models predicted next day closing price of NSEI 


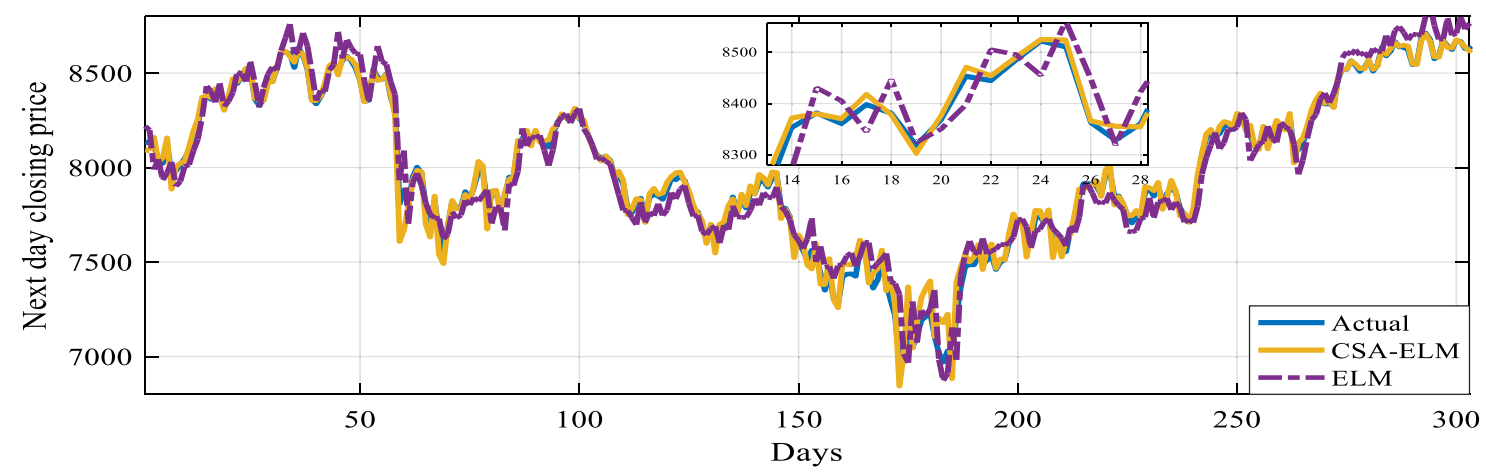

(a)

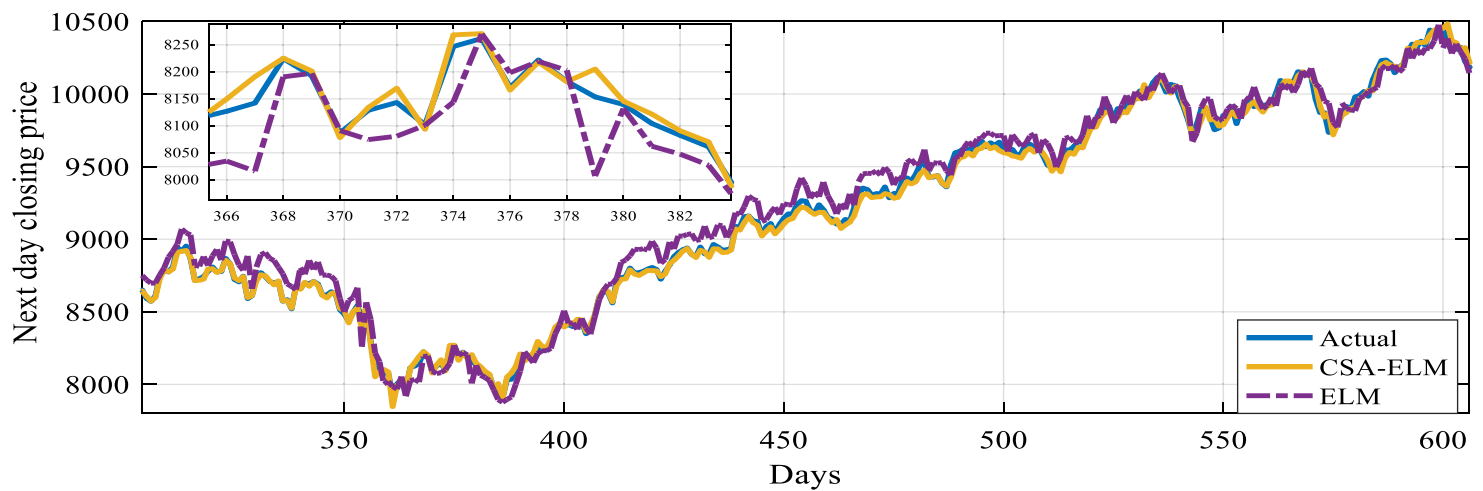

(b)

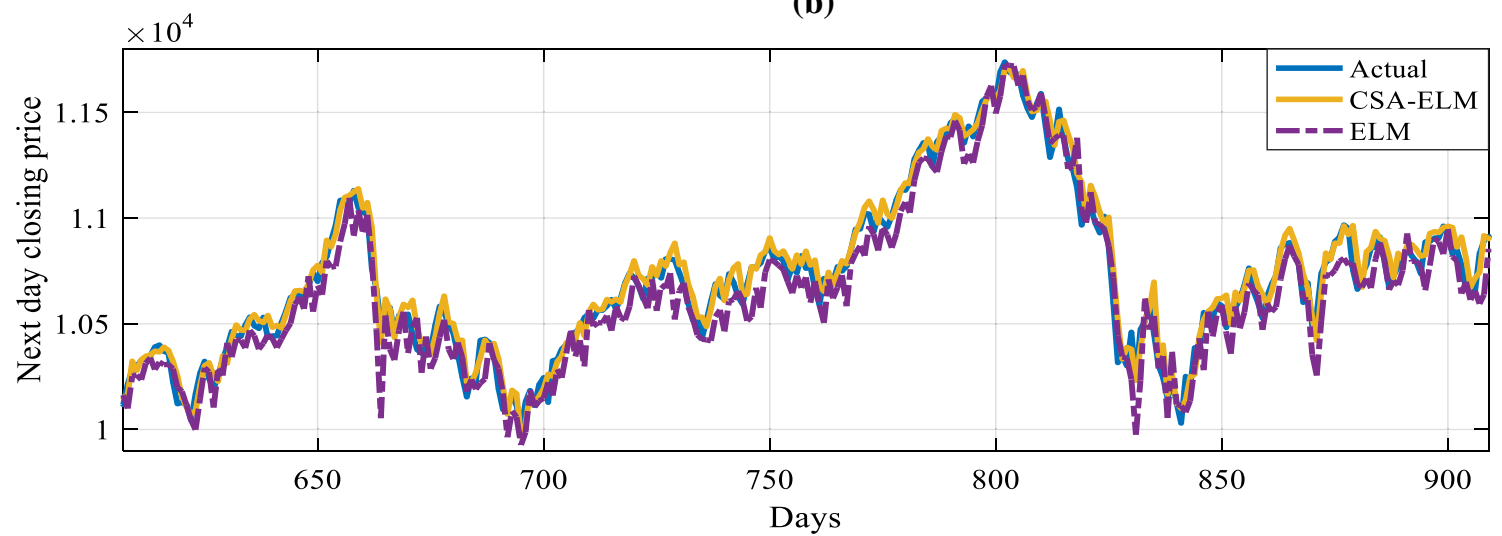

(c)

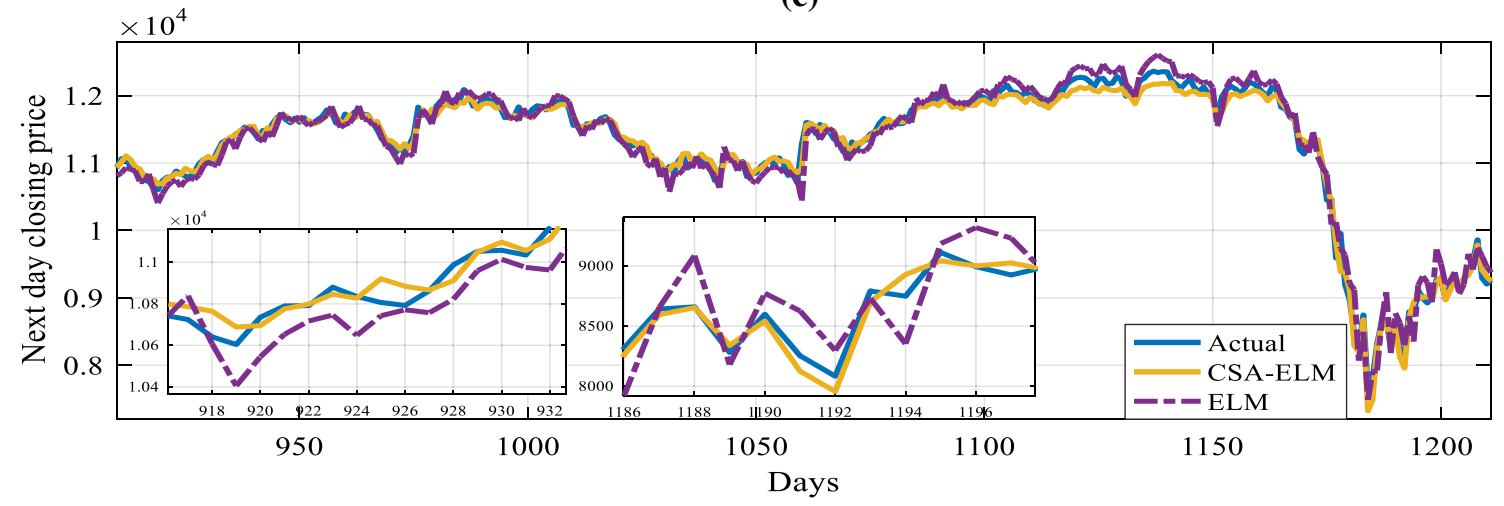

(d)

Fig. 21 CSA ELM and ELM models predicted next day closing price of NSEI 


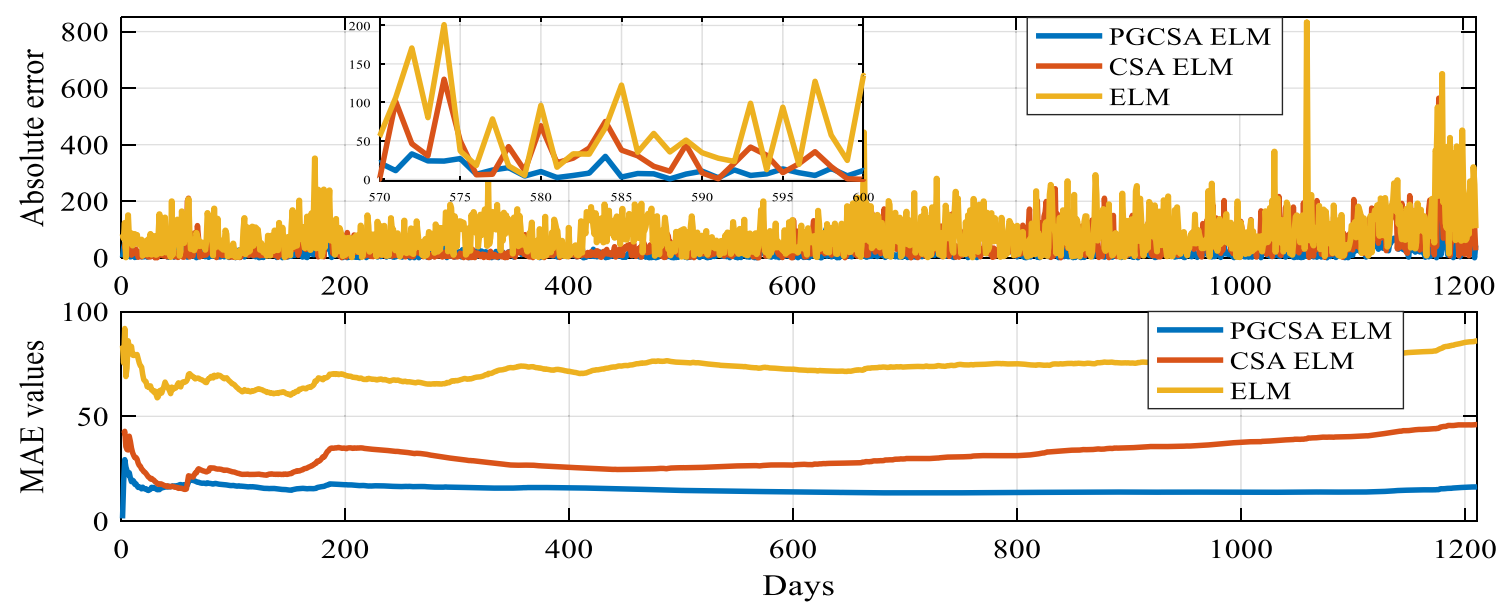

Fig. 22 Absolute error and MAE values of predicted NSEI closing price

\subsection{Simulation experiments of stock market forecasting}

For each stock index, CSA and PGCSA algorithm are executed individually with 100 populations to optimize ELM with ten neurons for 1000 iterations. The prime objective of optimization technique is to minimize Mean Squared Error (MSE). The competence of ELM model is influenced by the numbers of neurons of hidden layer. Initially, the ELM model is executed by varying the neurons of hidden layer. MSE, MAE and MAPE of ELM predicted closing price of IXIC are tabulated in Table 9 with different neurons. From Table 9, the CSA-ELM model with ten neurons is realized with better performance parameters. So, the ELM with ten hidden neurons is executed for all indices to predict closing price. The activation function of ELM is also a decisive factor by which the performance is influenced. The selection of relevant activation function for this work is done by a comparative analysis. The activation functions considered for the comparative analysis are sigmoid, hyperbolic tangent (tanh), softsign and rectified linear unit (ReLU) activation functions. The performance measures of forecasted closing price of IXIC with different activation functions are portrayed in Table 10. From Table 10, sigmoid activation function is concluded as a better activation function with better overall performance.

The performance parameters (MSE, MAE and MAPE) of stock indices with PGCSA-ELM, CSA-ELM and ELM models are tabulated in Table 11. PGCSA-ELM, CSAELM and ELM models predicted closing price with respect to actual closing price, absolute error, and MAE are portrayed in Figs. 8, 9, 10, 11, 12, 13, 14, 15, 16, 17, 18, 19, $20,21,22,23,24,25,26,27,28$. The testing results of different models are illustrated by splitting into four parts to portray a clear comparative analysis. The comparative analysis is portrayed to validate proposed PGCSA ELM over CSA ELM and CSA ELM over ELM in these figures. The zoomed part of predicted closing price is illustrated to contribute a fair comparative analysis. Figures 8, 9, 10, 11, $12,13,14,15,16,17,18,19,20,21,22,23,24,25,26,27$, 28 represent the performance of prediction to substantiate PGCSA ELM over CSA ELM and ELM model in terms of prediction of closing price. From Table 11 and Figs. 8, 9, $10,11,12,13,14,15,16,17,18,19,20,21,22,23,24,25$, 26, 27, 28, PGCSA-ELM model to predict SM closing price is favourably affirmed as a superior model in comparison with CSA-ELM, and ELM models.

Further, the comparison between actual closing price and predicted closing price in terms of statistical measures (MAAPE, CoV, CORR and Theil's U) is portrayed in Table 12. The PGCSA-ELM predicted model is substantiated with better performance measures in comparison to CSA-ELM and ELM models. From Table 12, PGCSAELM predicted closing price is substantiated as better forecast over CSA-ELM predicted closing price.

The computational time of ELM, CSA-ELM and PGCSA-ELM models evaluated during the training period is portrayed in Table 13. The weights and biases of ELM are evaluated by optimization techniques during training period and the evaluated parameters are fixed to test the prediction ability. So, the computational time is evaluated during training period. The increased computational complexity of the metaheuristic-based ELM models are observed to possess a higher computational time. However, the computational time can be compensated with a significant improvement in prediction performance.

The evidence to substantiate the integration of proposed PGCSA algorithm and optimized ELM has been depicted by simulation results as portrayed in previous sections. Seven different stock market indices are considered for the analysis. The improvement (in percentage) of 


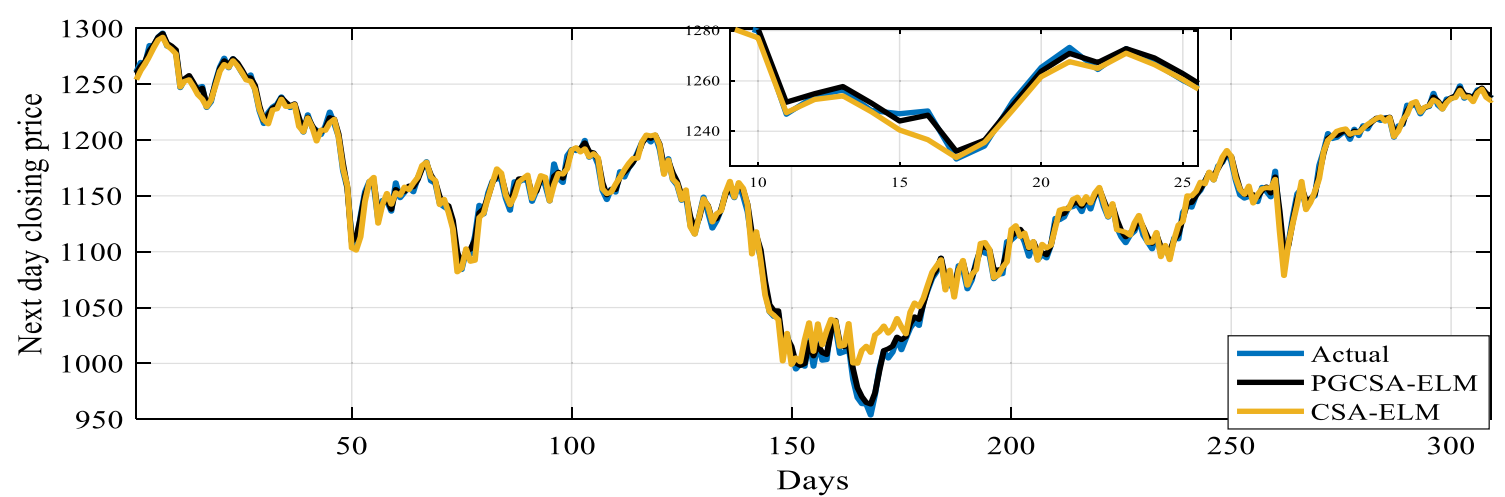

(a)

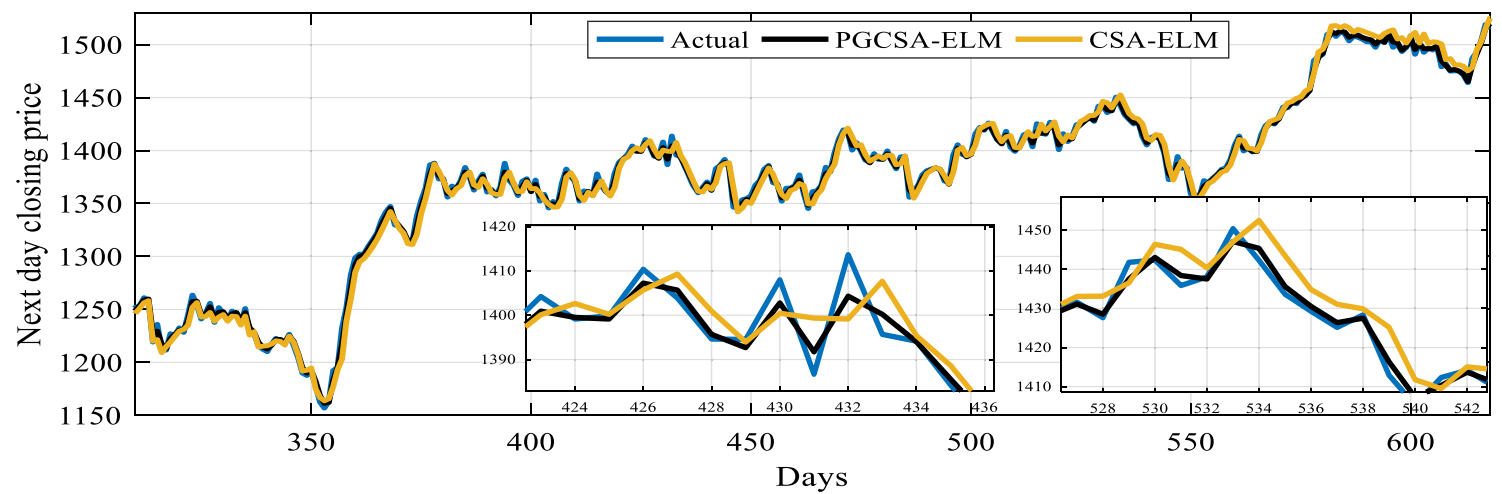

(b)

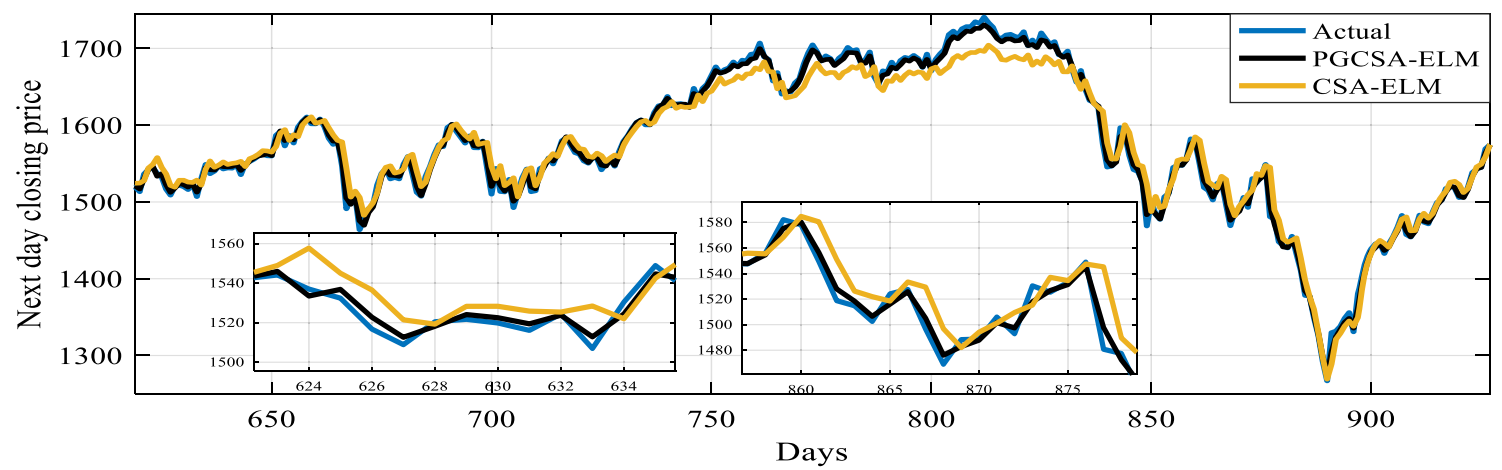

(c)

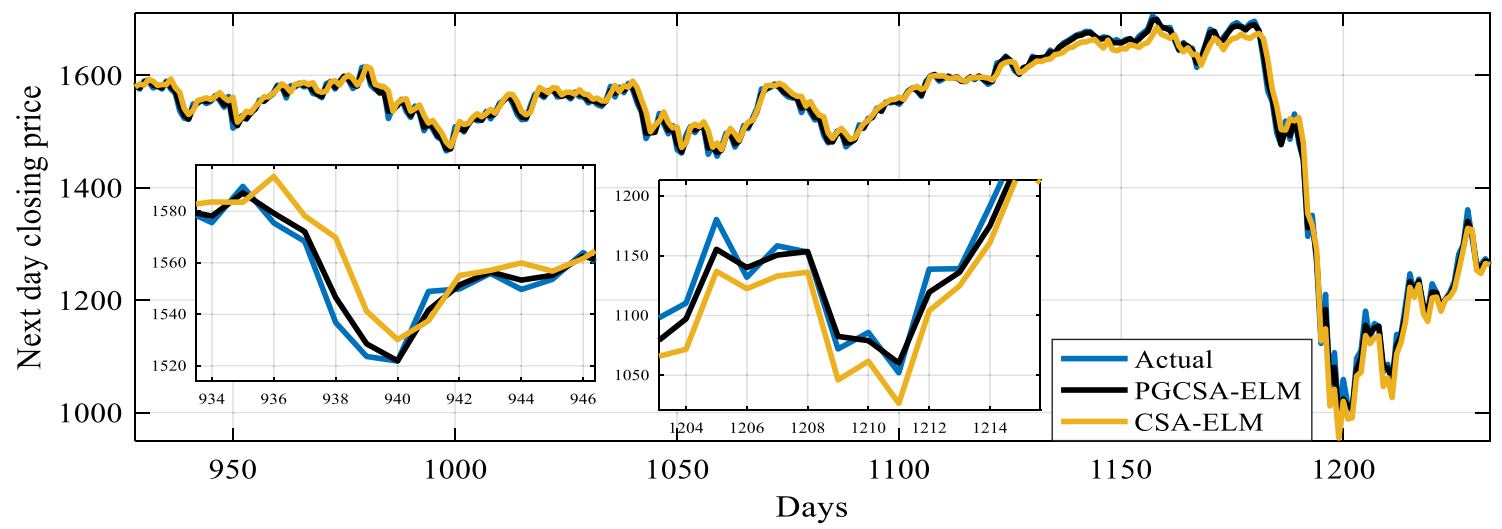

(d)

Fig. 23 PGCSA ELM and CSA ELM models predicted next day closing price of RUT 


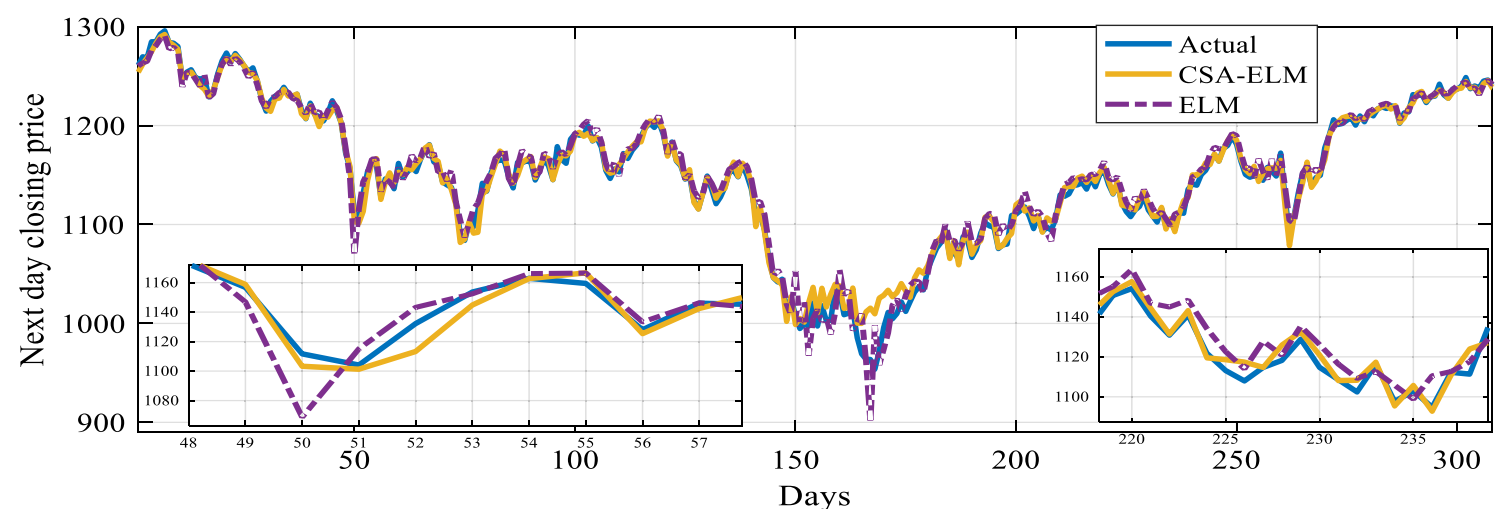

(a)

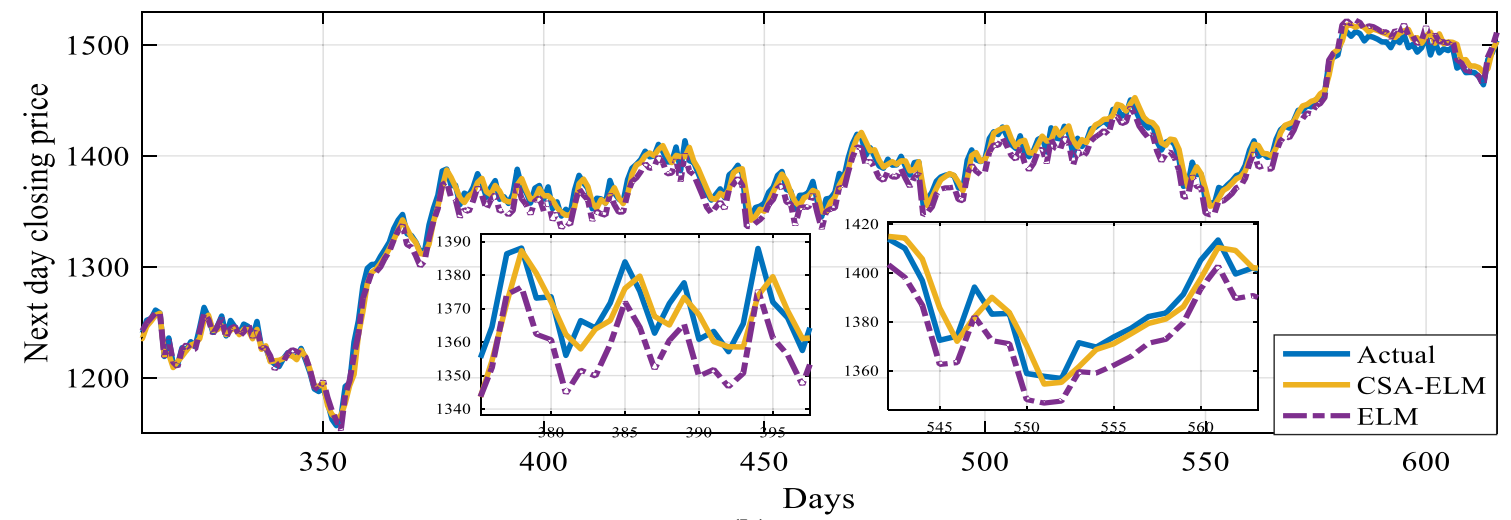

(b)

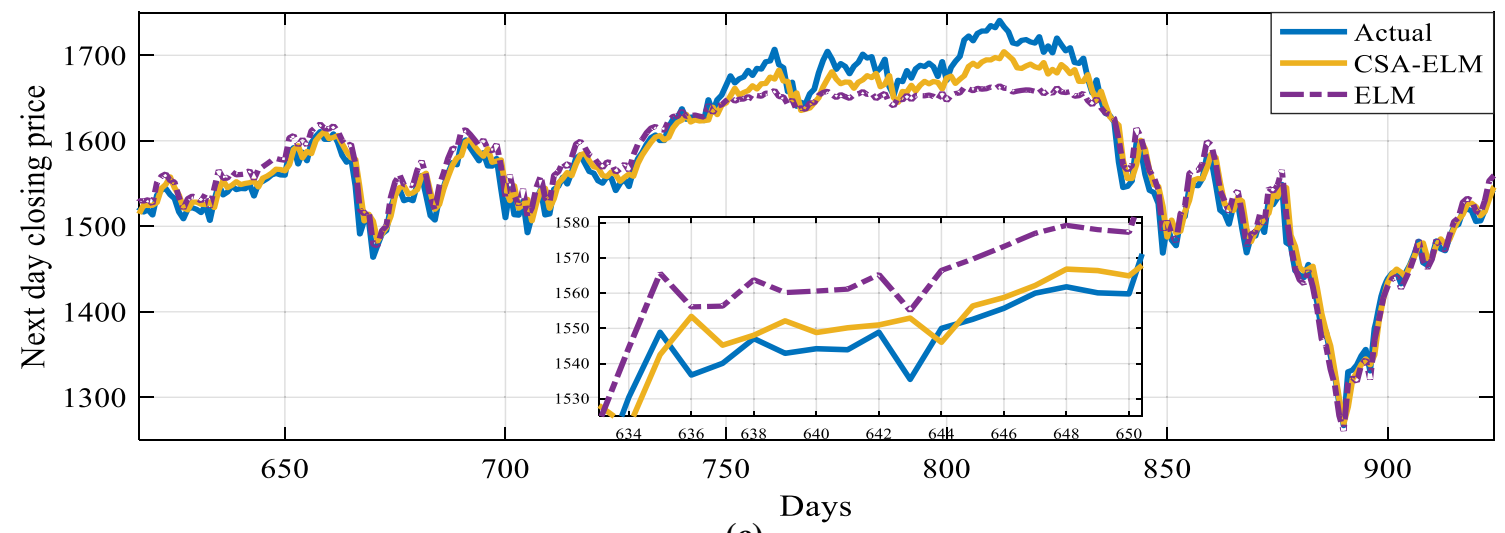

(c)

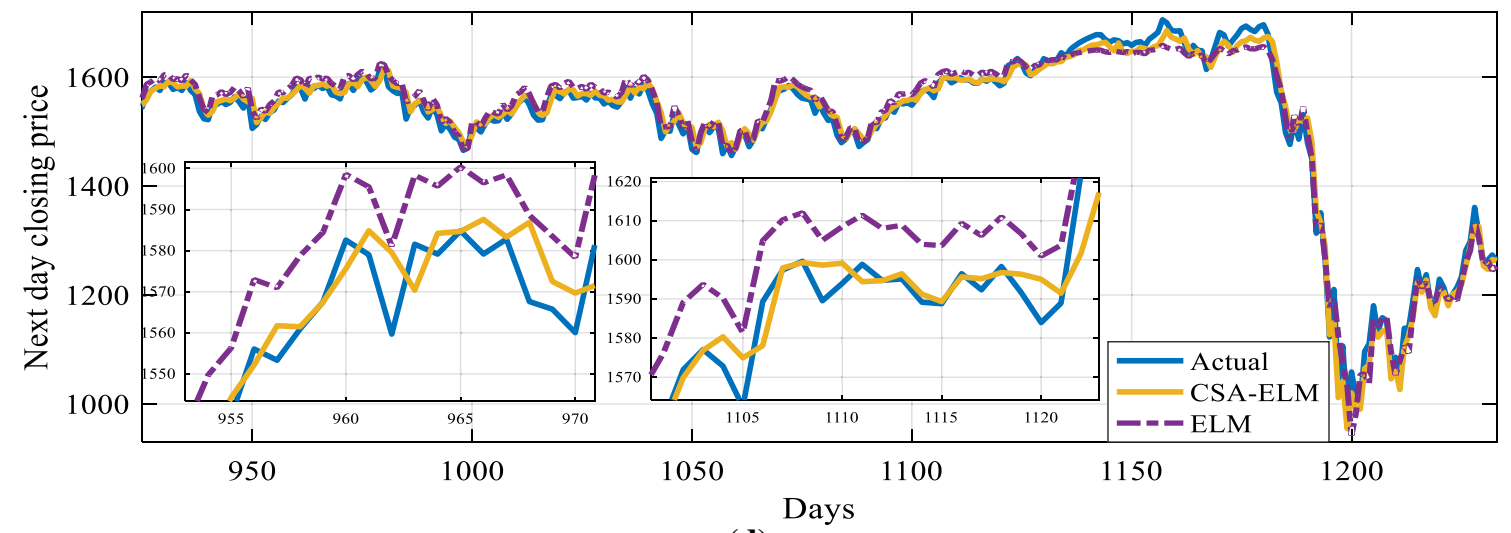

(d)

Fig. 24 CSA ELM and ELM models predicted next day closing price of RUT 


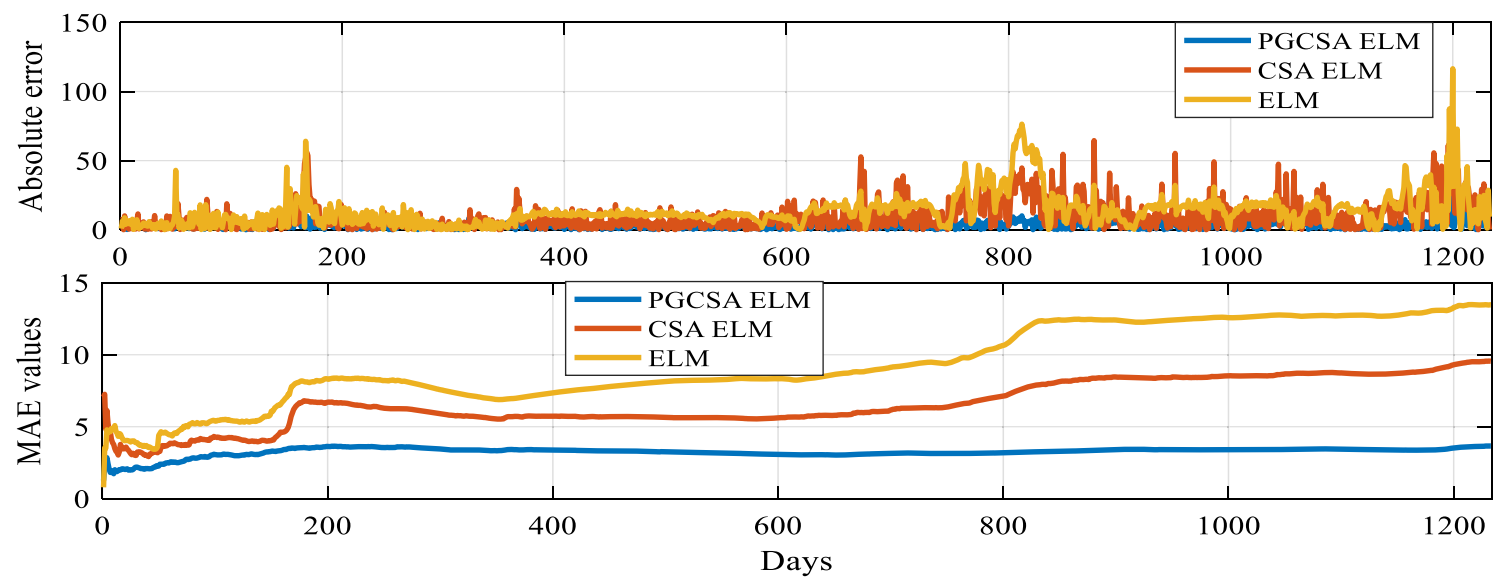

Fig. 25 Absolute error and MAE values of predicted NSEI closing price

performances in terms of measures of PGCSA ELM over CSA ELM and ELM methods is portrayed in Table 14.

\subsubsection{Verification of SM price prediction by Paired t-test}

The accuracy of prediction of closing price of SM is hypothetically tested by employing paired t-test. This test substantiates the proposed algorithm to contribute mean difference between predicted closing price and actual closing price. The statistical measure (paired t-test) requires two samples of observations of $n$ numbers population. In this work, actual closing price $\left(\mathrm{S}_{\mathrm{i}}\right)$ and predicted closing price $\left(\widehat{S_{i}}\right)$ are considered as two samples for paired t-test. The paired t-test results are tabulated in Table 15 . The significance level of 5\% (0.05) is adopted to accept the paired t-test. From Table 15, it is clearly realized $|\mathrm{t}|<\left|\mathrm{t}_{\text {critical }}\right|$ and $\rho>0.05$ for all seven markets. The absolute value of t-value contributed by proposed algorithm is less as compared with CSA-ELM and ELM models.

\subsubsection{Verification of SM price prediction by sharpe ratio and modified sharpe ratio}

In previous section, the predicted data administered by proposed method is hypothetically tested by paired t-test. In this section, the predicted closing price is tested through financial indicators such as annual return (AR), Sharpe ratio (SR) and modified sharpe ratio (MSR). AR is the yearly profit in percentage as defined in Eq. (31).

$A R=\left(\left(\frac{\text { Return }}{\text { capital }}\right)^{\frac{1}{n t}}-1\right) * 100$

where, 'nt' is the number of trading days of a year. The AR, SR and MSR of actual \& predicted closing price of stock markets have been portrayed in Table 16. These financial indicators of PGCSA-ELM predicted closing price are higher in comparison with CSA-ELM and ELM predicted closing price.

From Table 16, PGCSA-ELM predicted closing price is more sensitivity to non-normal distribution of return and financial indicators. PGCSA-ELM predicted price is close to the indicators of actual closing price of an index which means, the proposed model predicts price close enough to the actual trading cost.

\section{Conclusion}

Accurate forecasting of the stock markets price is the subject of extreme concern, especially in recent years. This study proposed a novel approach based on PGCSA and ELM for forecasting stock market closing price precisely. The proposed technique is also substantiated by including the data of seven indices during COVID-19 outbreak.

First, PGCSA algorithm is mainly based upon the awareness probability of a crow. Without awareness probability, the flock of crows are splited into different groups to explore the search space. The crows with awareness probability are updated with velocity by concerning each group's best crow. The proficiency of proposed PGCSA algorithm to solve 9 benchmark equations without constraints and 3 benchmark equations with constraints is substantiated over CSA, SSA, TLBO, PSO and $\mathrm{DE}$ algorithms. The effectiveness of proposed algorithm to resolve benchmark equations are acknowledged by conceding best value, mean and standard deviation as performance parameters. In addition to that, Wilcoxon test is considered for the hypothetical validation of the proposed approach.

Moreover, the CSA and proposed PGCSA algorithms are enforced individually to optimize the weight and bias of ELM to forecast next day closing price of seven different stock indices. MSE, MAE and MAPE are considered as 


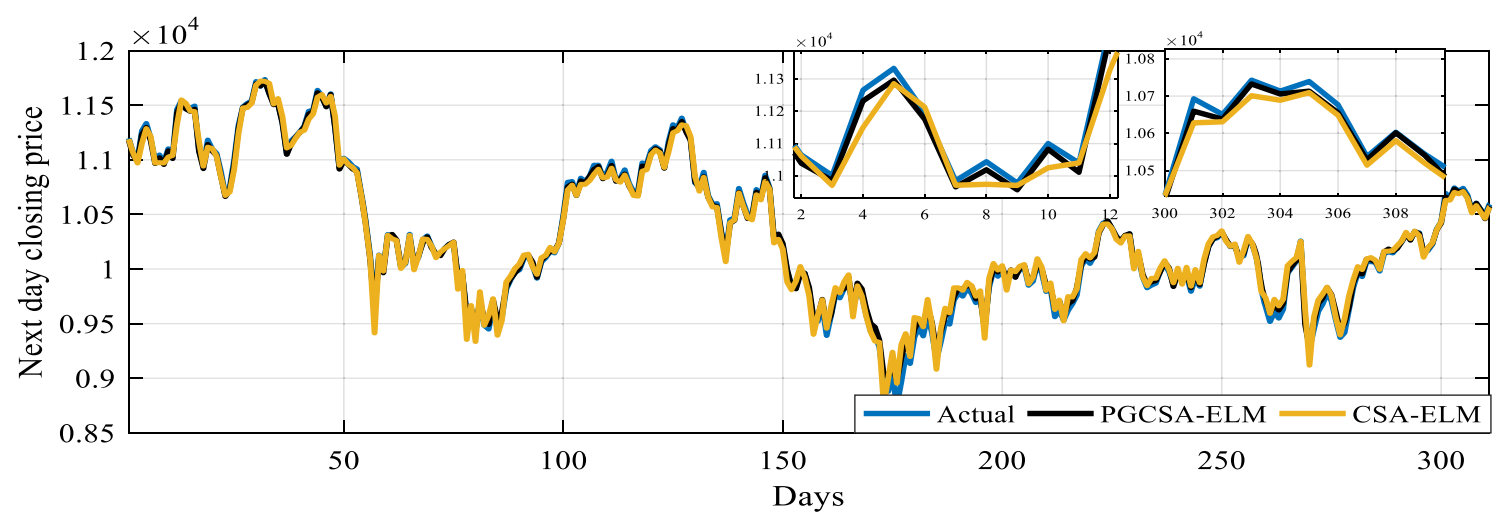

(a)

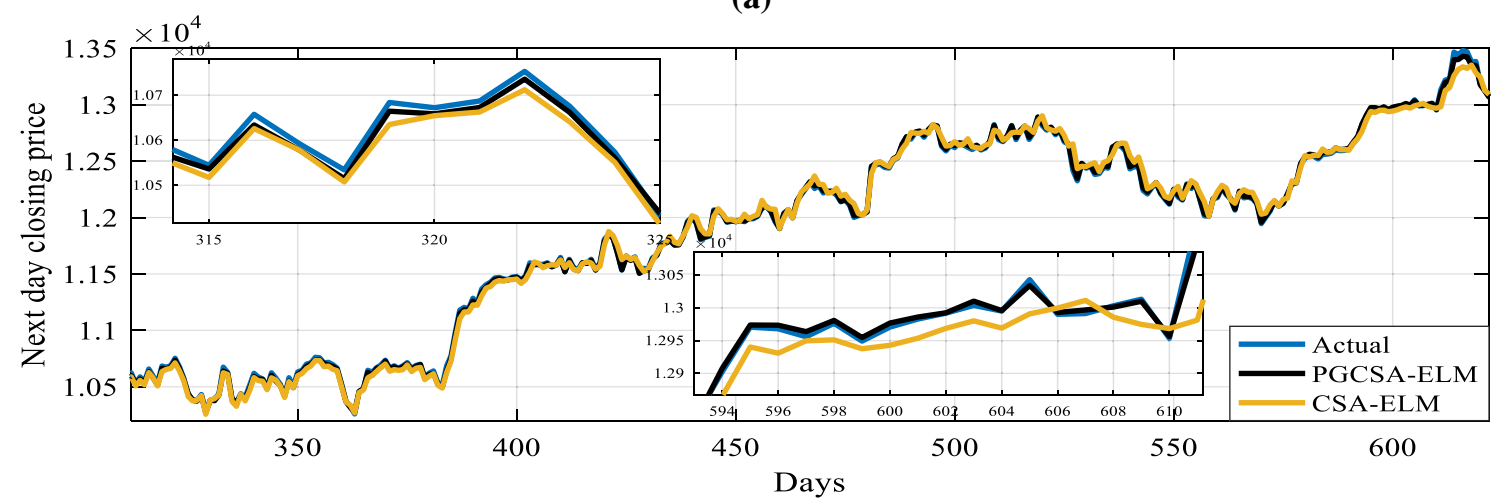

(b)

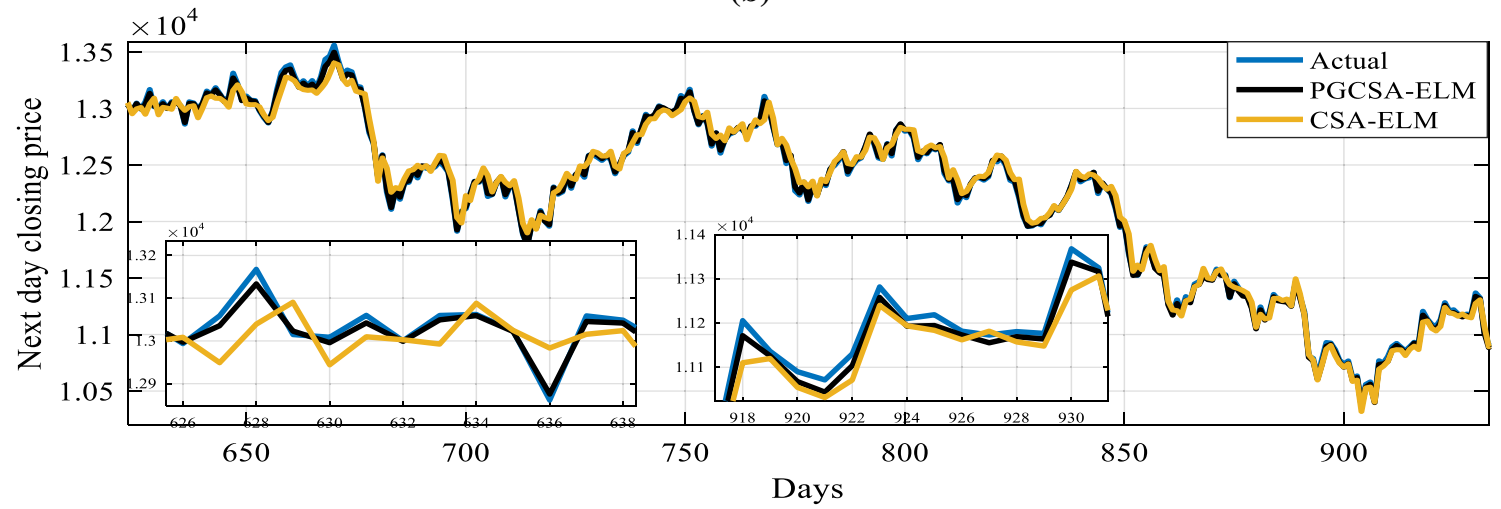

(c)

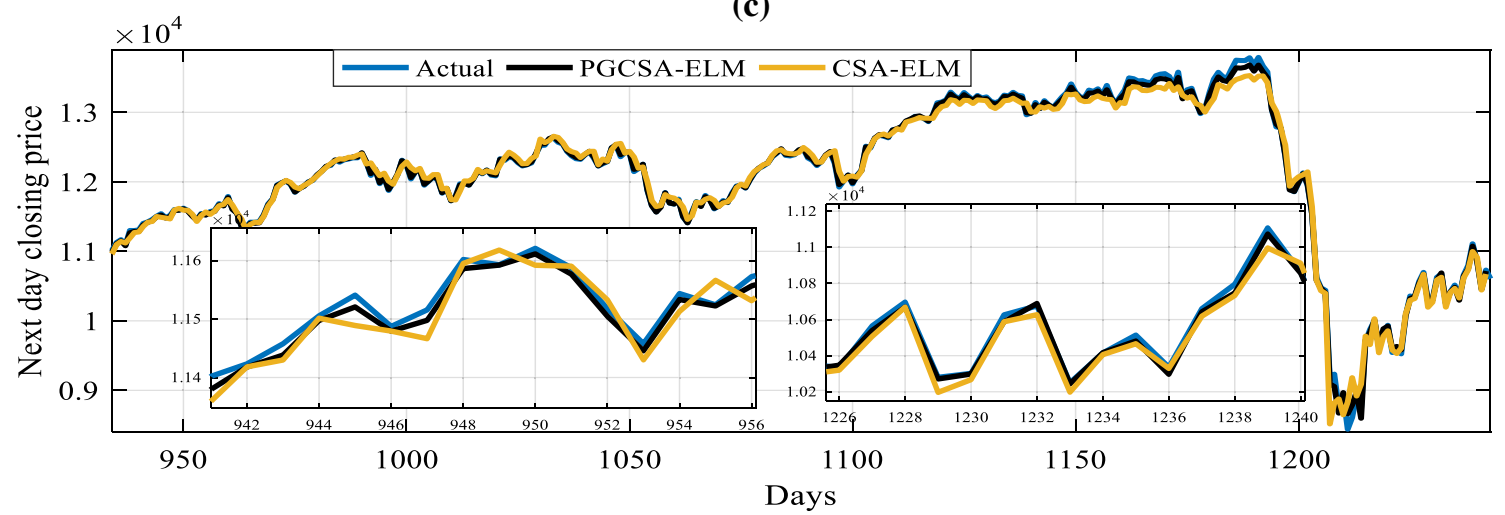

(d)

Fig. 26 PGCSA ELM and CSA ELM models predicted next day closing price of GDAXI 


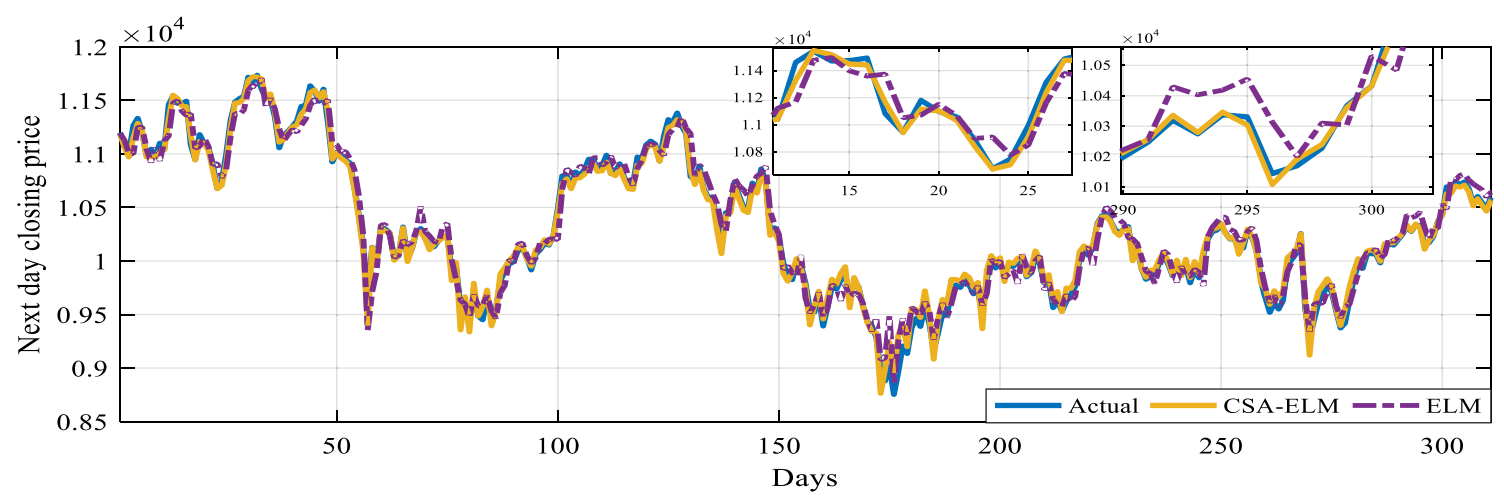

(a)

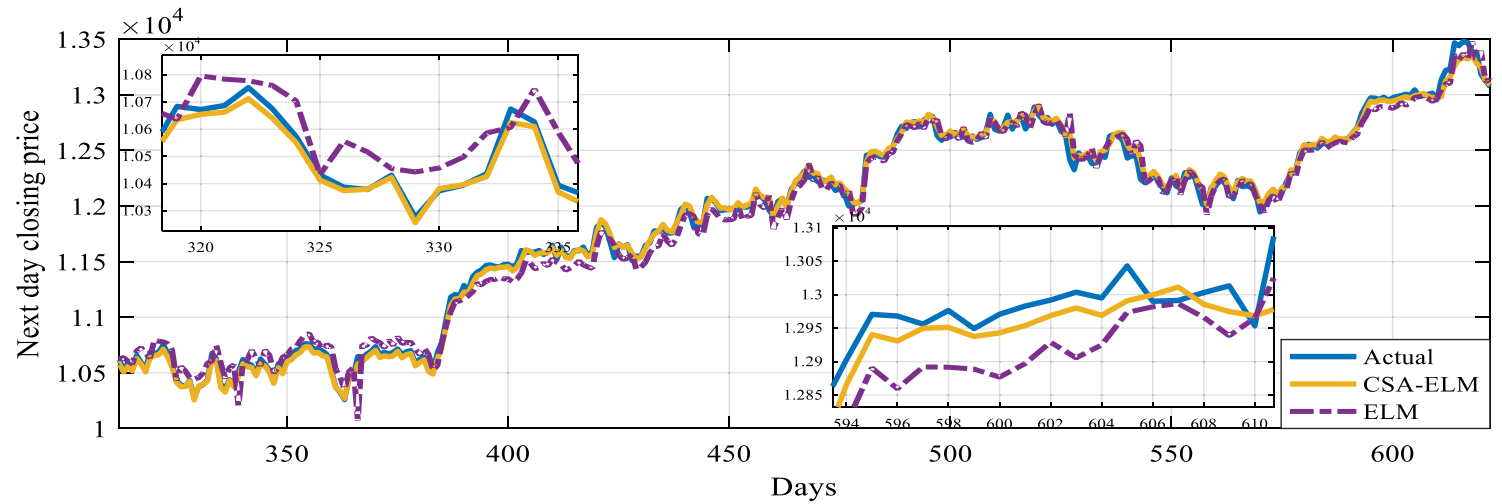

(b)

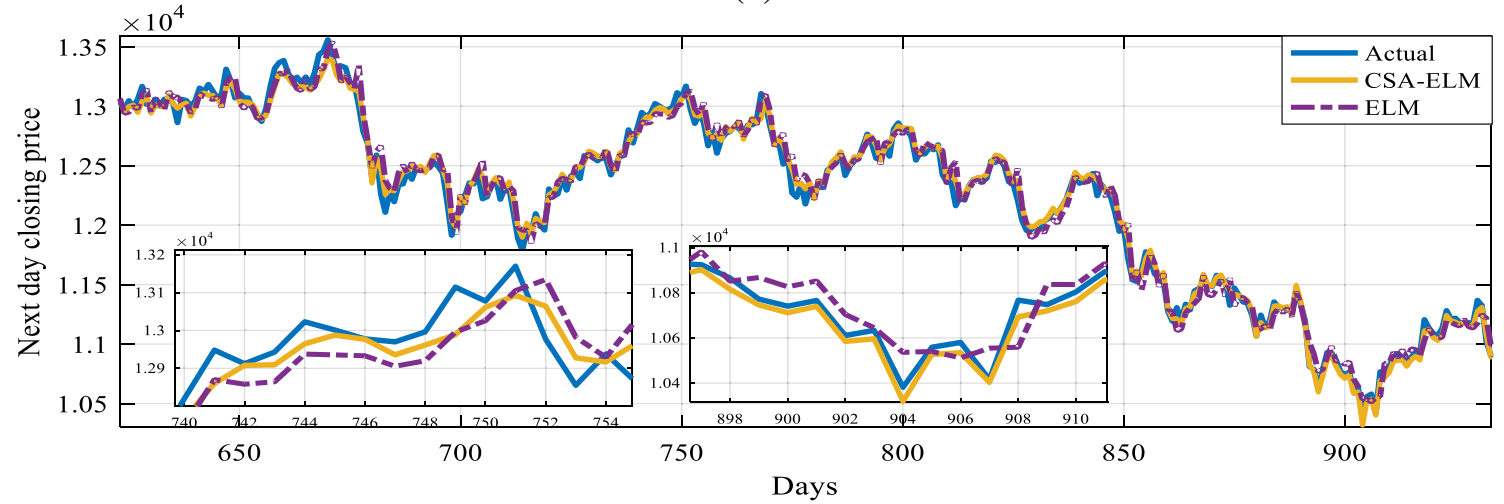

(c)

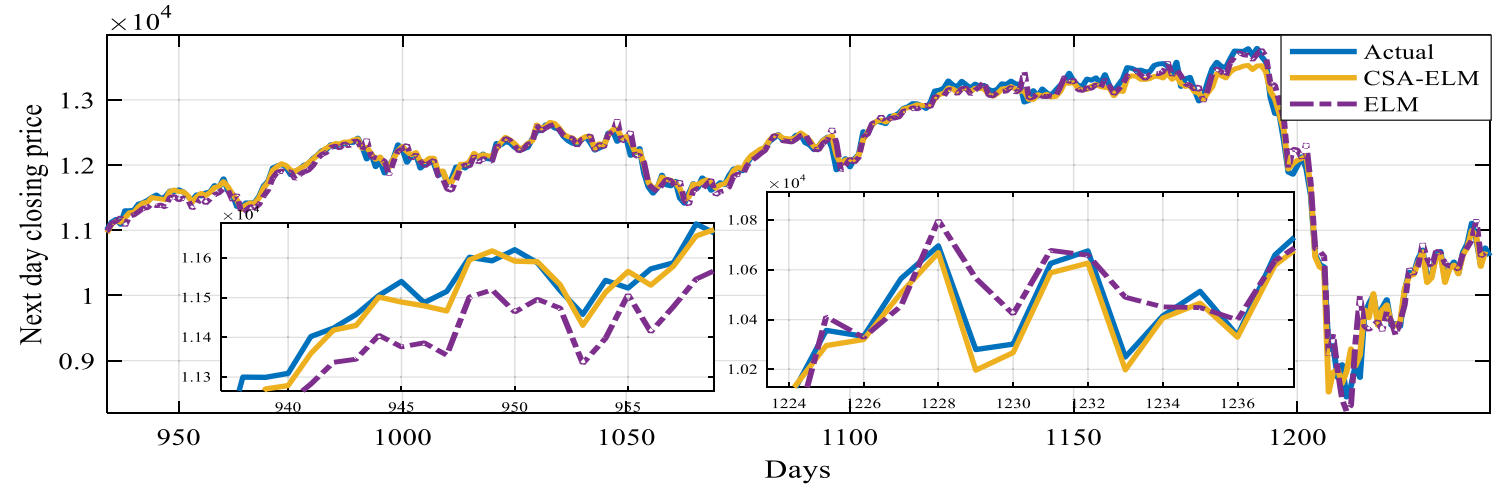

(d)

Fig. 27 CSA ELM and ELM models predicted next day closing price of GDAXI 

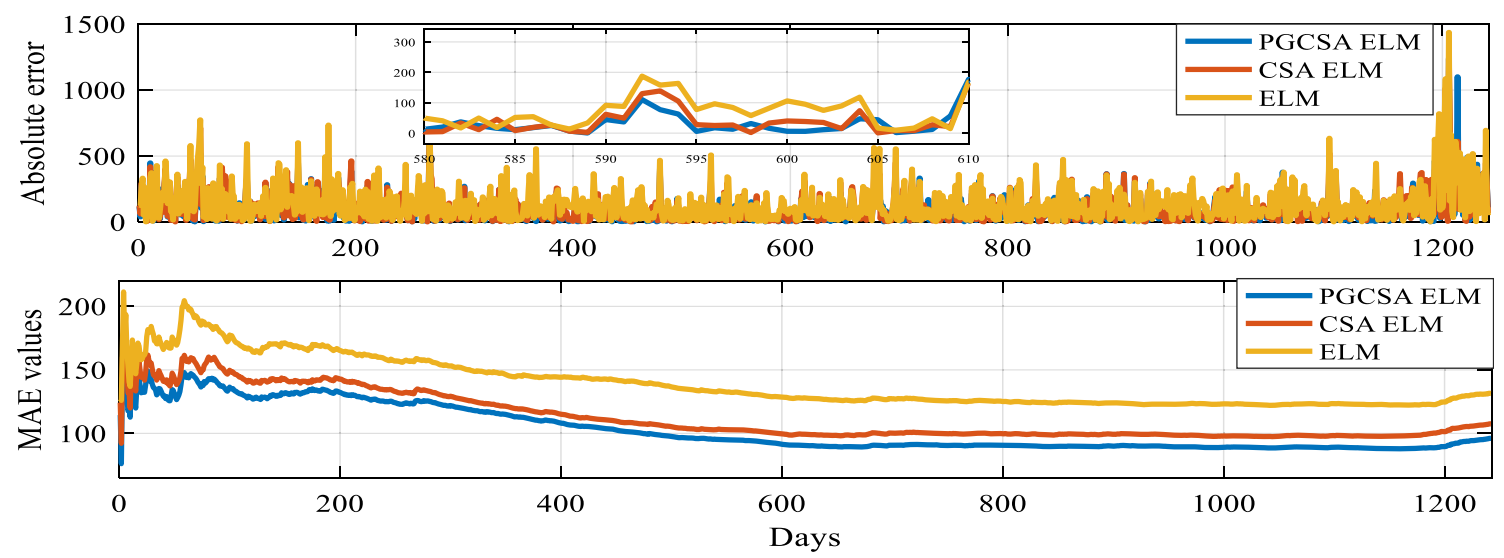

Fig. 28 Absolute error and MAE values of predicted GDAXI closing price

Table 12 Comparison of performance measures

\begin{tabular}{llllll}
\hline Markets & Prediction Model & MAAPE & CoV & CORR & Theil's U \\
\hline DJI & PGCSA-ELM & $\mathbf{0 . 0 0 1 9}$ & $\mathbf{1 6 . 7 9 8 1}$ & $\mathbf{0 . 9 9 9 8}$ & $\mathbf{0 . 0 0 1 6}$ \\
& CSA-ELM & 0.0043 & 16.7857 & 0.9993 & 0.0030 \\
\multirow{2}{*}{ HSI } & ELM & 0.0066 & 16.7645 & 0.9981 & 0.0051 \\
& PGCSA-ELM & $\mathbf{0 . 0 0 1 8}$ & $\mathbf{1 2 . 5 7 1 7}$ & $\mathbf{0 . 9 9 9 7}$ & $\mathbf{0 . 0 0 1 5}$ \\
& CSA-ELM & 0.0038 & 12.5696 & 0.9988 & 0.0029 \\
\multirow{2}{*}{ IXIC } & ELM & 0.0071 & 12.5443 & 0.9967 & 0.0050 \\
& PGCSA-ELM & $\mathbf{0 . 0 0 2 6}$ & $\mathbf{2 0 . 4 8 1 9}$ & 0.9996 & $\mathbf{0 . 0 0 2 4}$ \\
\multirow{3}{*}{ N100 } & CSA-ELM & 0.0039 & 20.4687 & 0.9997 & 0.0028 \\
& ELM & 0.0097 & 20.4437 & 0.9979 & 0.0064 \\
& PGCSA-ELM & $\mathbf{0 . 0 0 1 7}$ & $\mathbf{8 . 9 7 2 8}$ & $\mathbf{0 . 9 9 9 5}$ & $\mathbf{0 . 0 0 1 4}$ \\
NSEI & CSA-ELM & 0.0038 & 8.9537 & 0.9979 & 0.0028 \\
& ELM & 0.0062 & 8.9323 & 0.9949 & 0.0045 \\
& PGCSA-ELM & $\mathbf{0 . 0 0 1 7}$ & $\mathbf{1 4 . 7 3 0 3}$ & $\mathbf{0 . 9 9 9 8}$ & $\mathbf{0 . 0 0 1 2}$ \\
\multirow{2}{*}{ RUT } & CSA-ELM & 0.0045 & 14.7120 & 0.9988 & 0.0034 \\
& ELM & 0.0088 & 14.6778 & 0.9969 & 0.0057 \\
& PGCSA-ELM & $\mathbf{0 . 0 0 2 7}$ & $\mathbf{1 3 . 7 1 0 6}$ & $\mathbf{0 . 9 9 9 6}$ & $\mathbf{0 . 0 0 1 7}$ \\
\multirow{2}{*}{ GDAXI } & CSA-ELM & 0.0067 & 13.6755 & 0.9974 & 0.0048 \\
& ELM & 0.0094 & 13.6614 & 0.9957 & 0.0063 \\
& PGCSA-ELM & $\mathbf{0 . 0 0 1 7}$ & $\mathbf{9 . 7 9 3 9}$ & $\mathbf{0 . 9 9 9 7}$ & $\mathbf{0 . 0 0 1 2}$ \\
& CSA-ELM & 0.0044 & 9.7768 & 0.9978 & 0.0032 \\
& ELM & 0.0115 & 9.7398 & 0.9872 & 0.0078 \\
\hline
\end{tabular}

Bold values indicate the best value

Table 13 Computational time of different models in seconds

\begin{tabular}{llllllll}
\hline Models & DJI & HSI & IXIC & N100 & NSEI & RUT & GDAXI \\
\hline PGCSA ELM & 34.82 & 34.21 & 34.81 & 35.21 & 35.02 & 35.29 & 35.16 \\
CSA ELM & 34.51 & 34.11 & 34.51 & 35.16 & 34.77 & 35.11 & 34.57 \\
ELM & 8.74 & 9.23 & 9.47 & 8.64 & 8.41 & 9.02 & 8.79 \\
\hline
\end{tabular}


Table 14 Improvement of performance measures of PGCSA ELM in percentage

\begin{tabular}{lllllllll}
\hline Markets & Prediction Model & MSE & MAE & MAPE & MAAPE & CoV & CORR & Theil's U \\
\hline DJI & CSA-ELM & 70.79 & 55.52 & 54.91 & 55.81 & 0.07 & 0.05 & 46.66 \\
& ELM & 89.72 & 72.08 & 70.50 & 71.21 & 0.20 & 0.17 & 68.62 \\
HIS & CSA-ELM & 73.35 & 51.45 & 51.75 & 52.63 & 0.01 & 0.09 & 48.27 \\
& ELM & 90.83 & 74.49 & 73.77 & 74.64 & 0.21 & 0.3 & 70.00 \\
\multirow{2}{*}{ IXIC } & CSA-ELM & 22.84 & 34.52 & 33.43 & 33.33 & 0.06 & 0.01 & 14.28 \\
& ELM & 85.18 & 71.08 & 73.18 & 73.19 & 0.18 & 0.17 & 62.50 \\
\multirow{2}{*}{ N100 } & CSA-ELM & 75.04 & 54.93 & 54.05 & 55.26 & 0.21 & 0.16 & 50 \\
& ELM & 90.03 & 72.09 & 71.63 & 72.58 & 0.45 & 0.46 & 68.88 \\
\multirow{2}{*}{ NSEI } & CSA-ELM & 86.09 & 64.54 & 62.83 & 62.22 & 0.12 & 0.10 & 64.70 \\
& ELM & 95.00 & 81.01 & 80.75 & 80.68 & 0.35 & 0.29 & 78.94 \\
\multirow{2}{*}{ RUT } & CSA-ELM & 87.03 & 61.66 & 59.97 & 59.70 & 0.25 & 0.22 & 64.58 \\
& ELM & 92.21 & 72.81 & 71.31 & 71.27 & 0.36 & 0.39 & 73.01 \\
GDAXI & CSA-ELM & 85.13 & 5.28 & 61.77 & 61.36 & 0.17 & 0.19 & 62.50 \\
& ELM & 97.48 & 26.90 & 85.32 & 85.21 & 0.55 & 1.06 & 84.61 \\
\hline \multirow{2}{*}{} & & & & & & & &
\end{tabular}

Table 15 The paired t-test result

\begin{tabular}{|c|c|c|c|c|c|c|}
\hline Markets & Prediction Model & t-value & $\mathrm{t}_{\text {critical }}$ & $P$-value & Degree of freedom & Remark \\
\hline \multirow[t]{3}{*}{ DJI } & PGCSA-ELM & -0.0297 & \multirow[t]{3}{*}{ \pm 1.9618} & 0.9763 & \multirow[t]{3}{*}{1233} & \multirow{21}{*}{$\begin{array}{l}\text { Null hypotheses accepted } \\
\text { for all case studies }|t|<\left|t_{\text {critical }}\right|\end{array}$} \\
\hline & CSA-ELM & -0.0300 & & 0.9761 & & \\
\hline & ELM & -0.0308 & & 0.9754 & & \\
\hline \multirow[t]{3}{*}{ HIS } & PGCSA-ELM & 0.0166 & \multirow[t]{3}{*}{ \pm 1.9619} & 0.9867 & \multirow[t]{3}{*}{1205} & \\
\hline & CSA-ELM & 0.0177 & & 0.9859 & & \\
\hline & ELM & 0.0198 & & 0.9842 & & \\
\hline \multirow[t]{3}{*}{ IXIC } & PGCSA-ELM & -0.0582 & \multirow[t]{3}{*}{ \pm 1.9618} & 0.9536 & \multirow[t]{3}{*}{1233} & \\
\hline & CSA-ELM & -0.0586 & & 0.9533 & & \\
\hline & ELM & -0.0587 & & 0.9532 & & \\
\hline \multirow[t]{3}{*}{ N100 } & PGCSA-ELM & 0.0146 & \multirow[t]{3}{*}{ \pm 1.9618} & 0.9883 & \multirow[t]{3}{*}{1253} & \\
\hline & CSA-ELM & 0.0164 & & 0.9869 & & \\
\hline & ELM & 0.0203 & & 0.9838 & & \\
\hline \multirow[t]{3}{*}{ NSEI } & PGCSA-ELM & -0.0131 & \multirow[t]{3}{*}{ \pm 1.9619} & 0.9896 & \multirow[t]{3}{*}{1211} & \\
\hline & CSA-ELM & -0.0151 & & 0.9880 & & \\
\hline & ELM & 0.0155 & & 0.9876 & & \\
\hline \multirow[t]{3}{*}{ RUT } & PGCSA-ELM & -0.0011 & \multirow[t]{3}{*}{ \pm 1.9618} & 0.9991 & \multirow[t]{3}{*}{1233} & \\
\hline & CSA-ELM & -0.0037 & & 0.9970 & & \\
\hline & ELM & 0.0271 & & 0.9784 & & \\
\hline \multirow[t]{3}{*}{ GDAXI } & PGCSA-ELM & 0.0016 & \multirow[t]{3}{*}{ \pm 1.9618} & 0.9815 & \multirow[t]{3}{*}{1242} & \\
\hline & CSA-ELM & 0.0089 & & 0.9929 & & \\
\hline & ELM & 0.0073 & & 0.9942 & & \\
\hline
\end{tabular}

Bold values indicate the best value

Table 16 Comparison of financial indicators

\begin{tabular}{|c|c|c|c|c|c|c|c|c|c|c|c|c|}
\hline \multirow[t]{2}{*}{ Indices } & \multicolumn{3}{|c|}{ Actual closing price } & \multicolumn{3}{|c|}{ PGCSA ELM predicted price } & \multicolumn{3}{|c|}{ CSA ELM predicted price } & \multicolumn{3}{|c|}{ ELM predicted price } \\
\hline & AR & SR & MSR & $\mathrm{AR}$ & SR & MSR & $\mathrm{AR}$ & SR & MSR & AR & SR & MSR \\
\hline DJI & 0.6250 & 0.3966 & 0.5948 & 0.5793 & 0.4460 & 0.6380 & 0.6317 & 0.3909 & 0.5273 & 0.5384 & 0.3829 & 0.5050 \\
\hline HSI & -0.0837 & -0.0537 & 0.4976 & -0.0843 & -0.0542 & 0.4971 & -0.0758 & -0.0476 & 0.4835 & -0.0072 & -0.0035 & 0.3896 \\
\hline IXIC & 1.0859 & 0.6541 & 0.4748 & 1.0946 & 0.6303 & 0.4728 & 1.0491 & 0.7265 & 0.5474 & 1.0083 & 0.6027 & 0.4675 \\
\hline N100 & 0.0252 & 0.0174 & 0.5051 & 0.0358 & 0.0229 & 0.4923 & 0.0128 & 0.0086 & 0.4694 & 0.0829 & 0.0465 & 0.4597 \\
\hline NSEI & 0.5988 & 0.3909 & 0.5868 & 0.5842 & 0.4020 & 0.6151 & 0.5364 & 0.3797 & 0.5414 & 0.4528 & 0.3468 & 0.4844 \\
\hline RUT & 0.2142 & 1.1666 & 0.4290 & 0.1707 & 1.0754 & 0.4745 & 0.1372 & 0.9591 & 0.5137 & 0.1074 & 0.7026 & 0.5352 \\
\hline GDAXI & 0.1226 & 0.0753 & 0.4926 & 0.0994 & 0.0457 & 0.4626 & 0.0727 & 0.0581 & 0.4584 & 0.0358 & 0.0423 & 0.5657 \\
\hline
\end{tabular}


statistical weighs to contribute a fair comparative analysis to demonstrate the supremacy of PGCSA-ELM over CSAELM and ELM predicted models. Further, MAAPE, CoV, CORR and Theil's $U$ are used as statistical measures to substantiate PGCSA-ELM over CSA-ELM and ELM forecasting models with maximum CORR and minimum MAAPE, CoV \& Theil's U. From this work, it is corroborated that PGCSA-ELM forecasting model outperforms CSA-ELM and ELM forecasting models to predict next day closing price.
Finally, the mean difference between actual and forecasted closing price is hypothetically substantiated by adopting paired sample t-test. The risk adjusted basis relevant measures like AR, SR, MSR of actual and predicted closing price is also considered to achieve good return with less risk.

\section{Appendix}

See Table 17.

Table 17 Comparative analysis of GDAXI predicted value

\begin{tabular}{|c|c|c|c|c|c|c|c|c|}
\hline Actual & $\begin{array}{l}\text { PGCSA } \\
\text { ELM }\end{array}$ & $\begin{array}{l}\text { CSA } \\
\text { ELM }\end{array}$ & ELM & $\begin{array}{l}\text { Error of } \\
\text { PGCSA ELM } \\
\text { Model }\end{array}$ & $\begin{array}{l}\text { Error of CSA } \\
\text { ELM Model }\end{array}$ & $\begin{array}{l}\text { Error of } \\
\text { ELM } \\
\text { Model }\end{array}$ & $\begin{array}{l}\text { Win or Loss of PGCSA } \\
\text { ELM over CSA ELM }\end{array}$ & $\begin{array}{l}\text { Win or Loss of } \\
\text { PGCSA ELM over } \\
\text { ELM }\end{array}$ \\
\hline 3994.96 & 3994.95 & 3997.96 & 3994.94 & 0.0100 & 3.0044 & 0.0155 & + & + \\
\hline 4291.53 & 4291.25 & 4299.57 & 4291.54 & 0.2715 & 8.0429 & 0.0160 & + & - \\
\hline 4373.53 & 4373.50 & 4345.78 & 4373.02 & 0.0263 & 27.7429 & 0.5050 & + & + \\
\hline 4617.07 & 4617.20 & 4578.74 & 4617.84 & 0.1360 & 38.3218 & 0.7726 & + & + \\
\hline 5082.07 & 5080.73 & 5013.86 & 5081.85 & 1.3359 & 68.2045 & 0.2166 & + & - \\
\hline 5449.98 & 5382.94 & 5395.95 & 5317.32 & 67.03677 & 54.0245 & 132.6507 & - & + \\
\hline 6024.05 & 6005.86 & 5903.56 & 5952.88 & 18.1880 & 120.4836 & 71.1653 & + & + \\
\hline 5712.69 & 5792.81 & 5613.61 & 6330.08 & 80.1287 & 99.0789 & 617.3962 & + & + \\
\hline 5999.46 & 5998.24 & 5772.12 & 5777.31 & 1.2130 & 227.3399 & 222.1488 & + & + \\
\hline 6681.13 & 6316.24 & 6317.02 & 6244.96 & 364.8848 & 364.1007 & 436.1635 & - & + \\
\hline 6937.17 & 6608.13 & 6691.007 & 6638.62 & 329.0316 & 246.1626 & 298.5432 & - & - \\
\hline 7958.24 & 7795.44 & 7921.81 & 7774.88 & 162.7985 & 36.4263 & 183.3591 & - & + \\
\hline 7922.42 & 7934.86 & 8001.68 & 7922.46 & 12.4480 & 79.2670 & 0.0484 & + & - \\
\hline 7949.11 & 7954.50 & 8058.53 & 7949.14 & 5.3978 & 109.4239 & 0.0311 & + & - \\
\hline 6720.33 & 6702.97 & 6525.43 & 6720.37 & 17.3544 & 194.8961 & 0.0431 & + & - \\
\hline 6315.94 & 6675.48 & 6799.16 & 6666.11 & 359.5484 & 483.2279 & 350.1748 & + & - \\
\hline 5806.33 & 5898.74 & 5703.78 & 5590.71 & 92.4171 & 102.5419 & 215.619 & + & + \\
\hline 4973.07 & 5632.47 & 5322.44 & 5824.05 & 659.4067 & 349.3711 & 850.9858 & - & + \\
\hline 4131.07 & 4156.89 & 3987.19 & 4221.30 & 25.8212 & 143.8741 & 90.2388 & + & + \\
\hline 4905.44 & 4905.20 & 4896.99 & 4905.51 & 0.2099 & 8.447161 & 0.0702 & + & - \\
\hline 5554.55 & 5557.90 & 5504.41 & 5516.18 & 3.3556 & 50.1370 & 38.3643 & + & + \\
\hline 6048.3 & 6094.46 & 6078.93 & 5843.70 & 46.1691 & 30.6334 & 204.5911 & - & + \\
\hline 6235.56 & 6393.12 & 6380.95 & 6417.14 & 157.5647 & 145.3985 & 181.585 & - & + \\
\hline 5857.43 & 6034.26 & 6173.76 & 6476.56 & 176.8377 & 316.339 & 619.1399 & + & + \\
\hline 6211.34 & 6132.02 & 5683.61 & 5888.92 & 79.3136 & 527.7254 & 322.4199 & + & + \\
\hline 6989.74 & 6435.23 & 6520.31 & 6627.23 & 554.5076 & 469.425 & 362.5005 & - & - \\
\hline 7179.81 & 7245.89 & 7355.39 & 7391.08 & 66.0854 & 175.5832 & 211.2766 & + & + \\
\hline 7419.44 & 7410.90 & 7502.10 & 7423.29 & 8.5352 & 82.6620 & 3.8566 & + & - \\
\hline 5216.71 & 5216.64 & 5215.83 & 5216.75 & 0.0660 & 0.8761 & 0.0426 & + & - \\
\hline 6075.52 & 6124.44 & 5985.79 & 6255.77 & 48.9203 & 89.7295 & 180.2516 & + & + \\
\hline 7056.65 & 6760.442 & 6662.09 & 6514.37 & 296.2076 & 394.5538 & 542.2783 & + & + \\
\hline 6496.08 & 6529.27 & 6357.13 & 7000 & 33.1974 & 138.9411 & 503.92 & $\begin{array}{l}+ \\
\text { Wins(24) Loss (8) }\end{array}$ & $\begin{array}{l}+ \\
\text { Wins(21) Loss (11) }\end{array}$ \\
\hline
\end{tabular}

' + ' indicates wins and '-' indicates losses in comparative analysis 


\section{Declarations}

Conflict of interest The authors declare that they have no conflict of interest.

\section{References}

1. R Schabacker 2005 Technical analysis and stock market profits Harriman House Limited Petersfield

2. GE Box GM Jenkins GC Reinsel GM Ljung 2015 Time series analysis: forecasting and control John Wiley \& Sons

3. M O'Hara GS Oldfield 1986 The microeconomics of market making J Financ Quant Anal 214361376

4. CM Bilson TJ Brailsford VJ Hooper 2001 Selecting macroeconomic variables as explanatory factors of emerging stock market returns Pac Basin Financ J 94401426 https://doi.org/10.1016/ S0927-538X(01)00020-8

5. S Singh KS Parmar J Kumar 2021 Soft computing model coupled with statistical models to estimate future of stock market Neural Comput Appl https://doi.org/10.1007/s00521-020-05506-1

6. O Bustos A Pomares-Quimbaya 2020 Stock market movement forecast: a systematic review Expert Syst Appl 156113464 https://doi.org/10.1016/j.eswa.2020.113464

7. AH Moghaddam MH Moghaddam M Esfandyari 2016 Stock market index prediction using artificial neural network $\mathrm{J}$ Econ Financ Adm Sci 21418993 https://doi.org/10.1016/j.jefas.2016. 07.002

8. M Qiu Y Song F Akagi 2016 Application of artificial neural network for the prediction of stock market returns: the case of the Japanese stock market Chaos Solitons Fract 8517 https://doi.org/ 10.1016/j.chaos.2016.01.004

9. D Vukovic Y Vyklyuk N Matsiuk M Maiti 2020 Neural network forecasting in prediction Sharpe ratio: evidence from EU debt market Physica A 542123331 https://doi.org/10.1016/j.physa. 2019.123331

10. E Guresen G Kayakutlu TU Daim 2011 Using artificial neural network models in stock market index prediction Expert Syst Appl 3881038910397 https://doi.org/10.1016/j.eswa.2011.02. 068

11. S Gupta LP Wang 2010 Stock forecasting with feedforward neural networks and gradual data sub-sampling Aust J Intel Inf Process Syst 1141417

12. LO Orimoloye MC Sung T Ma JE Johnson 2020 Comparing the effectiveness of deep feedforward neural networks and shallow architectures for predicting stock price indices Expert Syst Appl 139112828 https://doi.org/10.1016/j.eswa.2019.112828

13. G Dudek 2020 Multilayer perceptron for short-term load forecasting: from global to local approach Neural Comput Appl 328 36953707 https://doi.org/10.1007/s00521-019-04130-y

14. JZ Wang JJ Wang ZG Zhang SP Guo 2011 Forecasting stock indices with back propagation neural network Expert Syst Appl 38111434614355 https://doi.org/10.1016/j.eswa.2011.04.222

15. D Zhang S Lou 2020 The application research of neural network and BP algorithm in stock price pattern classification and prediction Futur Gener Comput Syst 115872879 https://doi.org/10. 1016/j.future.2020.10.009

16. H Gunduz Y Yaslan Z Cataltepe 2017 Intraday prediction of Borsa Istanbul using convolutional neural networks and feature correlations Knowl-Based Syst 137138148 https://doi.org/10. 1016/j.knosys.2017.09.023

17. E Hoseinzade S Haratizadeh 2019 CNNpred: CNN-based stock market prediction using a diverse set of variables Expert Syst Appl 129273285 https://doi.org/10.1016/j.eswa.2019.03.029
18. C Cortes V Vapnik 1995 Support-vector networks Machine Learn 203273297 https://doi.org/10.1007/BF00994018

19. JD Wu CT Liu 2011 Finger-vein pattern identification using SVM and neural network technique Expert Syst Appl 3811 1428414289 https://doi.org/10.1016/j.eswa.2011.05.086

20. H Tang P Dong Y Shi 2019 A new approach of integrating piecewise linear representation and weighted support vector machine for forecasting stock turning points Appl Soft Comput 78685696 https://doi.org/10.1016/j.asoc.2019.02.039

21. X Li Y Sun 2020 Stock intelligent investment strategy based on support vector machine parameter optimization algorithm Neural Comput Appl 32617651775 https://doi.org/10.1007/s00521019-04566-2

22. M Nikou G Mansourfar J Bagherzadeh 2019 Stock price prediction using DEEP learning algorithm and its comparison with machine learning algorithms Intel Syst Account Financ Manag 26 4164174 https://doi.org/10.1002/isaf.1459

23. GB Huang QY Zhu CK Siew 2006 Extreme learning machine: theory and applications Neurocomputing 70 1-3 489501 https:// doi.org/10.1016/j.neucom.2005.12.126

24. Cheng GJ, Cai L, Pan HX (2009) Comparison of extreme learning machine with support vector regression for reservoir permeability prediction. In 2009 International Conference on Computational Intelligence and Security. IEEE. 2:173-176. Doi: https://doi.org/10.1109/CIS.2009.124

25. GB Huang H Zhou X Ding R Zhang 2011 Extreme learning machine for regression and multiclass classification IEEE Trans Syst Man Cybern Part B Cybern 422513529 https://doi.org/10. 1109/TSMCB.2011.2168604

26. X Li H Xie R Wang Y Cai J Cao F Wang H Min X Deng 2016 Empirical analysis: stock market prediction via extreme learning machine Neural Comput Appl 2716778 https://doi.org/10.1007/ s00521-014-1550-z

27. F Sun KA Toh MG Romay K Mao 2014 Extreme learning machines: algorithms and applications Springer International Publishing Berlin

28. E Hadavandi H Shavandi A Ghanbari 2010 Integration of genetic fuzzy systems and artificial neural networks for stock price forecasting Knowl-Based Syst 238800808 https://doi.org/10. 1016/j.knosys.2010.05.004

29. R Choudhry K Garg 2008 A hybrid machine learning system for stock market forecasting World Acad Sci Eng Technol 393315 318

30. Y Perwej A Perwej 2012 Prediction of the Bombay Stock Exchange (BSE) market returns using artificial neural network and genetic algorithm J Intell Learn Syst Appl 42108119 https://doi.org/10.4236/jilsa.2012.42010

31. H Chung KS Shin 2020 Genetic algorithm-optimized multichannel convolutional neural network for stock market prediction Neural Comput Appl 321278977914 https://doi.org/10.1007/ s00521-019-04236-3

32. XD Zhang A Li R Pan 2016 Stock trend prediction based on a new status box method and AdaBoost probabilistic support vector machine Appl Soft Comput 49385398 https://doi.org/10.1016/j. asoc.2016.08.026

33. Hegazy O, Soliman OS, Salam MA (2014) A machine learning model for stock market prediction. arXiv preprint ar Xiv:1402. 7351. 4(12):17-23

34. AK Rout B Biswal PK Dash 2014 A hybrid FLANN and adaptive differential evolution model for forecasting of stock market indices Int J Knowl based Intell Eng Syst 1812341 https://doi. org/10.3233/KES-130283

35. W Shen X Guo C Wu D Wu 2011 Forecasting stock indices using radial basis function neural networks optimized by artificial fish swarm algorithm Knowl-Based Syst 243378385 https://doi.org/ 10.1016/j.knosys.2010.11.001 
36. SK Chandar 2021 Grey Wolf optimization-Elman neural network model for stock price prediction Soft Comput 251649658 https://doi.org/10.1007/s00500-020-05174-2

37. A Kazem E Sharifi FK Hussain M Saberi OK Hussain 2013 Support vector regression with chaos-based firefly algorithm for stock market price forecasting Appl Soft Comput 132947958 https://doi.org/10.1016/j.asoc.2012.09.024

38. T Xiong Y Bao Z Hu R Chiong 2015 Forecasting interval time series using a fully complex-valued RBF neural network with DPSO and PSO algorithms Inf Sci 3057792 https://doi.org/10. 1016/j.ins.2015.01.029

39. Worasucheep C (2015) Forecasting currency exchange rates with an Artificial Bee Colony-optimized neural network. In 2015 IEEE Congress on Evolutionary Computation (CEC). IEEE. 3319-3326. doi:https://doi.org/10.1109/CEC.2015.7257305

40. G Dhiman V Kumar 2017 Spotted hyena optimizer: a novel bioinspired based metaheuristic technique for engineering applications Adv Eng Softw 1144870 https://doi.org/10.1016/j. advengsoft.2017.05.014

41. DE Goldberg JH Holland 1988 Genetic algorithms and machine learning Mach Learn 329599

42. R Storn K Price 1997 Differential evolution-a simple and efficient heuristic for global optimization over continuous spaces $\mathbf{J}$ Global Optim $11 \quad 4 \quad 341 \quad 359$ https://doi.org/10.1023/A: 1008202821328

43. E Rashedi H Nezamabadi-Pour S Saryazdi 2009 GSA: a gravitational search algorithm Inf Sci 1791322322248 https://doi. org/10.1016/j.ins.2009.03.004

44. Eberhart R, Kennedy J (1995) A new optimizer using particle swarm theory. In MHS'95. Proceedings of the Sixth International Symposium on Micro Machine and Human Science. IEEE. 39-43. doi: https://doi.org/10.1109/MHS.1995.494215.

45. Karaboga D (2005) An idea based on honey bee swarm for numerical optimization. Technical report-tr06, Erciyes university, engineering faculty, computer engineering department. 200:1-10

46. S Saremi S Mirjalili A Lewis 2017 Grasshopper optimisation algorithm: theory and application Adv Eng Softw 1053047 https://doi.org/10.1016/j.advengsoft.2017.01.004

47. MY Cheng D Prayogo 2014 Symbiotic organisms search: a new metaheuristic optimization algorithm Comput Struct 13998112 https://doi.org/10.1016/j.compstruc.2014.03.007

48. A Askarzadeh 2016 A novel metaheuristic method for solving constrained engineering optimization problems: crow search algorithm Comput Struct 169112 https://doi.org/10.1016/j. compstruc.2016.03.001
49. Sayed GI, Darwish A, Hassanien AE (2017) Chaotic crow search algorithm for engineering and constrained problems. In 2017 12th International Conference on Computer Engineering and Systems (ICCES). IEEE. 676-681. doi:https://doi.org/10.1109/ICCES. 2017.8275390.

50. GI Sayed AE Hassanien AT Azar 2019 Feature selection via a novel chaotic crow search algorithm Neural Comput Appl 311 171188 https://doi.org/10.1007/s00521-017-2988-6

51. F Mohammadi H Abdi 2018 A modified crow search algorithm (MCSA) for solving economic load dispatch problem Appl Soft Comput 715165 https://doi.org/10.1016/j.asoc.2018.06.040

52. AE Hassanien RM Rizk-Allah M Elhoseny 2018 A hybrid crow search algorithm based on rough searching scheme for solving engineering optimization problems $\mathbf{J}$ Ambient Intell Humaniz Comput https://doi.org/10.1007/s12652-018-0924-y

53. Pasandideh SHR., Khalilpourazari S (2018) Sine cosine crow search algorithm: a powerful hybrid meta heuristic for global optimization. arXiv preprint ar Xiv:1801. 08485.

54. R Hafezi J Shahrabi E Hadavandi 2015 A bat-neural network multi-agent system (BNNMAS) for stock price prediction: case study of DAX stock price Appl Soft Comput $29196210 \mathrm{https}: / /$ doi.org/10.1016/j.asoc.2014.12.028

55. Atashpaz-Gargari E, Lucas C (2007) Imperialist competitive algorithm: an algorithm for optimization inspired by imperialistic competition. In 2007 IEEE congress on evolutionary computation. IEEE. 4661-4667. doi:https://doi.org/10.1109/CEC.2007. 4425083

56. S Kim H Kim 2016 A new metric of absolute percentage error for intermittent demand forecasts Int J Forecast 323669679 https:// doi.org/10.1016/j.ijforecast.2015.12.003

57. GN Gregoriou JP Gueyie 2003 Risk-adjusted performance of funds of hedge funds using a modified Sharpe ratio $\mathrm{J}$ Wealth Manag 637783 https://doi.org/10.3905/jwm.2003.442378

58. RV Rao VJ Savsani DP Vakharia 2011 Teaching-learning-based optimization: a novel method for constrained mechanical design optimization problems Comput Aided Des 433303315 https:// doi.org/10.1016/j.cad.2010.12.015

59. S Mirjalili AH Gandomi SZ Mirjalili S Saremi H Faris SM Mirjalili 2017 Salp swarm algorithm: a bio-inspired optimizer for engineering design problems Adv Eng Softw 114163191 https:// doi.org/10.1016/j.advengsoft.2017.07.002

Publisher's Note Springer Nature remains neutral with regard to jurisdictional claims in published maps and institutional affiliations. 